University of Tennessee Health Science Center

UTHSC Digital Commons

\title{
A Novel Distractive and Mobility-Enabling Lumbar Spinal Orthosis for Treating Mechanical Low Back Pain
}

Daniel Clay Hillyard

University of Tennessee Health Science Center

Follow this and additional works at: https://dc.uthsc.edu/dissertations

Part of the Other Analytical, Diagnostic and Therapeutic Techniques and Equipment Commons, and the Therapeutics Commons

\section{Recommended Citation}

Hillyard, Daniel Clay (http://orcid.org/0000-0002-3154-4444), "A Novel Distractive and Mobility-Enabling Lumbar Spinal Orthosis for Treating Mechanical Low Back Pain" (2017). Theses and Dissertations (ETD). Paper 428. http://dx.doi.org/10.21007/etd.cghs.2017.0438.

This Dissertation is brought to you for free and open access by the College of Graduate Health Sciences at UTHSC Digital Commons. It has been accepted for inclusion in Theses and Dissertations (ETD) by an authorized administrator of UTHSC Digital Commons. For more information, please contact jwelch30@uthsc.edu. 


\title{
A Novel Distractive and Mobility-Enabling Lumbar Spinal Orthosis for Treating Mechanical Low Back Pain
}

\author{
Abstract \\ Introduction: Lumbar spinal orthoses (LSOs) are often used as non-surgical treatment and serve to \\ support the spine and alleviate low back pain. More recently, dynamic orthoses claiming to decompress \\ the spine have been introduced. Currently, there is an unserved population of people that suffer from \\ mechanical low back pain (LBP) conditions, such as degenerative disc disease or lumbar foraminal \\ stenosis, that would benefit from spinal decompression and mobility. A previously-developed prototype of \\ dynamic mobility orthosis (DMO1) was designed that provided a distractive load across the lumbar spine \\ but required higher sagittal bending moments and was unable to maintain spinal off-loading throughout \\ extended ranges of movement. The research objectives were to a) Design a new orthosis that reduced \\ bending moment build up and sustained spinal off-loading throughout daily living ranges of flexion and \\ extension movement, b) Test the new orthosis prototype in a controlled laboratory environment, and c) \\ Organize and carry out a clinical pilot study with patients suffering from mechanical LBP to determine the \\ immediate and short-term effects of the new orthosis prototype on LBP and overall patient quality of life. \\ Methods: A mechanical analog upper torso model and programmable robotic testing platform were used \\ to design features of the new prototype (DMO2): a mobility-enabling component (MEC) and a distractive \\ force component (DFC). The DMO2 prototype was tested in a robotic testing platform (RTP) under a 300 \\ $\mathrm{N}$ applied vertical torso load over a range of $25^{\circ}$ flexion to $10^{\circ}$ of extension utilizing a previously- \\ developed protocol. For DMO2, loads carried by the brace were determined throughout flexion and \\ extension. Applied moments to upper torso model and transferred moments to spine were measured. The \\ difference in applied and transferred moments represented brace moment effects. It was determined that \\ DMO2 had limitations, primarily with providing a distractive force to actual human subjects. Because of \\ this, a new orthosis prototype (DMO3) was developed that improved upon the design of the DMO2 MEC \\ and DFC. The DMO3 prototype was designed to provide a constant distractive force with minimal \\ resistance to bending while effectively providing a distractive force to the wearer that could be felt. Also, \\ the MEC of DMO3 included both flexion and extension as well as axial rotation. The DMO3 prototype was \\ tested in the RTP under a $150 \mathrm{~N}$ applied vertical load over a range of $25^{\circ}$ flexion to $10^{\circ}$ of extension \\ utilizing a previously-developed protocol. Also, the DMO3 prototype was tested in the RTP under \\ simulated axial rotation without an applied vertical torso load. For this test, a measurement was made \\ that determined how much axial rotation $\mathrm{DMO} 3$ allowed. A clinical study was organized in which two \\ patients with LBP wore the DMO3 prototype during six physical therapy (PT) treatment sessions. Before \\ the treatment sessions began, the patient had a radiograph (x-ray 1$)$ taken and the patient completed a \\ Modified Oswestry Disability Questionnaire (mODI). During each treatment session, a pain score was \\ taken before wearing DMO3 and after completing exercises for 30-40 minutes while wearing the DMO. \\ Additionally, on the last treatment session, a radiograph ( $x$-ray 2 ) was taken. The lumbar disc height at the \\ diseased and adjacent lumbar levels were measured on $x$-ray 1 and $x$-ray 2 and compared. Also, the mODI \\ scores were compared before and after treatment. Results: The DMO2 prototype improved spinal off- \\ loading capacity from $172 \mathrm{~N}$ to $290 \mathrm{~N}$ at end range flexion and from $247 \mathrm{~N}$ to $293 \mathrm{~N}$ at end range \\ extension compared to the original DM01 prototype. End range applied moments (flexion-DM01: $32.4 \mathrm{Nm}$ \\ / DM02: 21.7 Nm; extension-DM01: 15.0 Nm / DMO2: 10.9 Nm) and brace moments (flexion DM01: 18.6 \\ Nm / DM02: $6.6 \mathrm{Nm}$; extension-DM01: $15.0 \mathrm{Nm} /$ DMO2: 4.4 Nm) were also reduced. The DMO3 \\ prototype was able to support $100 \%$ (at vertical stance), $104 \%$ (at end range flexion), and $97 \%$ (at end \\ range extension) of the applied vertical torso load during simulated flexion and extension. Also, the DMO3 \\ prototype contributed 0 of the $4.1 \mathrm{Nm}$ (at end range flexion), and 4.4 of the $6.6 \mathrm{Nm}$ (at end range \\ extension) of the total bending moment. During simulated axial rotation, the DMO3 prototype was able to \\ achieve 10 degrees of axial rotation in both the clockwise and counterclockwise directions. The DMO3 \\ prototype immediately reduced pain for both patient $1(p<0.001)$ and patient $2(p=0.005)$ during all PT
}


treatment sessions that they participated in. Patient 1 showed an of $2 \mathrm{~mm}$ (resolution $0.14 \mathrm{~mm}$ ) of lumbar disc height at the L5-S1 level between the start and end of the treatments with DMO3. Similarly, patient 2 showed an increase of $0.5 \mathrm{~mm}$ at both the L4-L5 and L5-S1 levels. Patient 1 and patient 2 showed a decrease in mODI scores from $70 \%$ to $62 \%$ and from $46 \%$ to $22 \%$, respectfully. Discussion: An advanced testing assembly was used in this study to carry out the design of two prototypes of a novel dynamic spinal orthosis having the unique design goal of providing spinal offloading while enabling mobility. Two prototype models (DMO2 and DMO3) were built and tested under simulated DLAs in a controlled laboratory environment. Throughout the development of the DMO, each prototype demonstrated significant improvements compared to its predecessor. The means by which the DMO3 prototype produced a distractive force allowed the force output of the DMO to remain constant even with minor fluctuations in vertical displacement as might be experienced in normal flexion and extension motions. Preliminary testing found DMO3 prototype completely removed all pain from patient 1 at five of her six PT treatment visits. Conclusion: A novel dynamic spinal orthosis was designed that maintained spinal off-loading throughout extended ranges of flexion and extension movement without buildup of adverse bending moments. Additionally, the novel orthosis was able to achieve enough axial rotation required for most daily living activities (DLAs). Preliminary testing found this orthosis was also effective on human subjects as pain was reduced during all PT treatment sessions for both patients. An orthosis that provides distraction and mobility has not been designed and the proposed spinal orthosis provided the first evidence of its clinical effectiveness on human subjects. Because of the efficacy of this orthosis, a larger clinical study is warranted to see if the DMO can have short-term and long-term effects on mechanical LBP.

\section{Document Type}

Dissertation

Degree Name

Doctor of Philosophy (PhD)

\section{Program}

Biomedical Engineering

\section{Research Advisor}

Denis J. DiAngelo, Ph.D.

\section{Keywords}

Biomechanical testing, Low back pain, Lumbar spinal orthosis

\section{Subject Categories}

Analytical, Diagnostic and Therapeutic Techniques and Equipment | Medicine and Health Sciences | Other Analytical, Diagnostic and Therapeutic Techniques and Equipment | Therapeutics

\section{Comments}

One year embargo expires May 2018. 


\title{
A Novel Distractive and Mobility-Enabling Lumbar Spinal Orthosis for Treating Mechanical Low Back Pain
}

\author{
A Thesis \\ Presented for \\ The Graduate Studies Council \\ The University of Tennessee \\ Health Science Center
}

\author{
In Partial Fulfillment \\ Of the Requirements for the Degree \\ Master of Science \\ In the Joint Graduate Program in Biomedical Engineering \\ From The University of Tennessee and \\ The University of Memphis
}

By

Daniel Clay Hillyard

May 2017 
Portions of Chapters 1-5 by SAGE Publishing. All other material (C 2017 by Daniel Clay Hillyard. All rights reserved. 


\section{DEDICATION}

This research is dedicated to both of my loving parents and to my Lord and Savior, Jesus Christ. The endless love, encouragement, and sacrifice that I have received from them throughout my life has granted me the opportunity to make a positive difference in the world.

"Nothing in this world can take the place of persistence. Talent will not; nothing is more common than unsuccessful men with talent. Genius will not; unrewarded genius is almost a proverb. Education will not; the world is full of educated derelicts. Persistence and determination along are omnipotent. The slogan "press on' has solved and will always solve the problems of the human race."

- Calvin Coolidge 


\section{ACKNOWLEDGEMENTS}

There are many people that I would like to take the time to express my sincere appreciation to that made great contributions to me research. I would like to thank my research advisor, Dr. Denis DiAngelo for his patience and support throughout my time here in the BioRobotics Laboratory; my research would have been possible without his guidance. I would also like to thank my committee for their encouragement, advice, and guidance. Dr. Carlos Rivera has provided valuable clinical insight and has been instrumental in helping advance this research into a clinical setting. Dr. John Simmons taught me much of what I know about the lab and the equipment in it. His contribution of designing the first orthosis prototype and developing the lab testing protocol laid the foundation for my research. Daniel Wido assisted in programming the robotic testing platform. Hunter Smith also taught me how to use some of the lab equipment during my time as an intern which I used as a graduate student in the lab. Chloe Chung was a great asset to have in the lab and assisted me with help and advice on many day-to-day tasks. Michael Parker and Cody Bateman assisted me in problem-solving and testing the medical device in a controlled laboratory environment. I am grateful for the opportunity that the Summer Research Scholars Program (sponsored by the College of Graduate Health Sciences at UTHSC) provided for me which ultimately contributed to me being added to the personnel of the BioRobotics Laboratory. The work in this research was possible because of the following: the robotic testing platform, previously-established protocols by the BioRobotics Laboratory in the Department of Orthopaedic Surgery and Biomedical Engineering, and the clinical equipment and facilities provided by Campbell Clinic Orthopaedics - Spine Center. Thank you to each and every one of you. 


\begin{abstract}
Introduction: Lumbar spinal orthoses (LSOs) are often used as non-surgical treatment and serve to support the spine and alleviate low back pain. More recently, dynamic orthoses claiming to decompress the spine have been introduced. Currently, there is an unserved population of people that suffer from mechanical low back pain (LBP) conditions, such as degenerative disc disease or lumbar foraminal stenosis, that would benefit from spinal decompression and mobility. A previously-developed prototype of dynamic mobility orthosis (DMO1) was designed that provided a distractive load across the lumbar spine but required higher sagittal bending moments and was unable to maintain spinal off-loading throughout extended ranges of movement. The research objectives were to a) Design a new orthosis that reduced bending moment build up and sustained spinal off-loading throughout daily living ranges of flexion and extension movement, b) Test the new orthosis prototype in a controlled laboratory environment, and c) Organize and carry out a clinical pilot study with patients suffering from mechanical LBP to determine the immediate and short-term effects of the new orthosis prototype on LBP and overall patient quality of life.
\end{abstract}

Methods: A mechanical analog upper torso model and programmable robotic testing platform were used to design features of the new prototype (DMO2): a mobility-enabling component (MEC) and a distractive force component (DFC). The DMO2 prototype was tested in a robotic testing platform (RTP) under a $300 \mathrm{~N}$ applied vertical torso load over a range of $25^{\circ}$ flexion to $10^{\circ}$ of extension utilizing a previously-developed protocol. For DMO2, loads carried by the brace were determined throughout flexion and extension. Applied moments to upper torso model and transferred moments to spine were measured. The difference in applied and transferred moments represented brace moment effects.

It was determined that DMO2 had limitations, primarily with providing a distractive force to actual human subjects. Because of this, a new orthosis prototype (DMO3) was developed that improved upon the design of the DMO2 MEC and DFC. The DMO3 prototype was designed to provide a constant distractive force with minimal resistance to bending while effectively providing a distractive force to the wearer that could be felt. Also, the MEC of DMO3 included both flexion and extension as well as axial rotation. The DMO3 prototype was tested in the RTP under a $150 \mathrm{~N}$ applied vertical load over a range of $25^{\circ}$ flexion to $10^{\circ}$ of extension utilizing a previously-developed protocol. Also, the DMO3 prototype was tested in the RTP under simulated axial rotation without an applied vertical torso load. For this test, a measurement was made that determined how much axial rotation DMO3 allowed.

A clinical study was organized in which two patients with LBP wore the DMO3 prototype during six physical therapy (PT) treatment sessions. Before the treatment sessions began, the patient had a radiograph (x-ray 1) taken and the patient completed a Modified Oswestry Disability Questionnaire (mODI). During each treatment session, a pain score was taken before wearing DMO3 and after completing exercises for 30-40 minutes while wearing the DMO. Additionally, on the last treatment session, a 
radiograph (x-ray 2) was taken. The lumbar disc height at the diseased and adjacent lumbar levels were measured on x-ray 1 and x-ray 2 and compared. Also, the mODI scores were compared before and after treatment.

Results: The DMO2 prototype improved spinal off-loading capacity from $172 \mathrm{~N}$ to 290 $\mathrm{N}$ at end range flexion and from $247 \mathrm{~N}$ to $293 \mathrm{~N}$ at end range extension compared to the original DMO1 prototype. End range applied moments (flexion-DMO1: $32.4 \mathrm{Nm} /$ DMO2: 21.7 Nm; extension-DMO1: 15.0 Nm / DMO2: 10.9 Nm) and brace moments (flexion DMO1: 18.6 Nm / DMO2: 6.6 Nm; extension-DMO1: 15.0 Nm / DMO2: 4.4 $\mathrm{Nm})$ were also reduced.

The DMO3 prototype was able to support 100\% (at vertical stance), 104\% (at end range flexion), and 97\% (at end range extension) of the applied vertical torso load during simulated flexion and extension. Also, the DMO3 prototype contributed 0 of the $4.1 \mathrm{Nm}$ (at end range flexion), and 4.4 of the $6.6 \mathrm{Nm}$ (at end range extension) of the total bending moment. During simulated axial rotation, the DMO3 prototype was able to achieve 10 degrees of axial rotation in both the clockwise and counterclockwise directions.

The DMO3 prototype immediately reduced pain for both patient $1(\mathrm{p}<0.001)$ and patient $2(\mathrm{p}=0.005)$ during all PT treatment sessions that they participated in. Patient 1 showed an of $2 \mathrm{~mm}$ (resolution $0.14 \mathrm{~mm}$ ) of lumbar disc height at the L5-S1 level between the start and end of the treatments with DMO3. Similarly, patient 2 showed an increase of 0.5 $\mathrm{mm}$ at both the L4-L5 and L5-S1 levels. Patient 1 and patient 2 showed a decrease in mODI scores from $70 \%$ to $62 \%$ and from $46 \%$ to $22 \%$, respectfully.

Discussion: An advanced testing assembly was used in this study to carry out the design of two prototypes of a novel dynamic spinal orthosis having the unique design goal of providing spinal offloading while enabling mobility. Two prototype models (DMO2 and DMO3) were built and tested under simulated DLAs in a controlled laboratory environment. Throughout the development of the DMO, each prototype demonstrated significant improvements compared to its predecessor. The means by which the DMO3 prototype produced a distractive force allowed the force output of the DMO to remain constant even with minor fluctuations in vertical displacement as might be experienced in normal flexion and extension motions. Preliminary testing found DMO3 prototype completely removed all pain from patient 1 at five of her six PT treatment visits.

Conclusion: A novel dynamic spinal orthosis was designed that maintained spinal offloading throughout extended ranges of flexion and extension movement without buildup of adverse bending moments. Additionally, the novel orthosis was able to achieve enough axial rotation required for most daily living activities (DLAs). Preliminary testing found this orthosis was also effective on human subjects as pain was reduced during all PT treatment sessions for both patients. An orthosis that provides distraction and mobility has not been designed and the proposed spinal orthosis provided the first evidence of its clinical effectiveness on human subjects. Because of the efficacy of this orthosis, a larger clinical study is warranted to see if the DMO can have short-term and long-term effects on mechanical LBP. 


\section{TABLE OF CONTENTS}



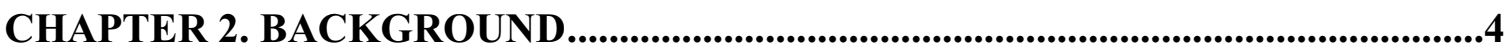

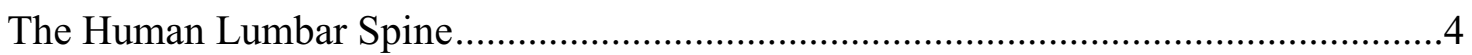

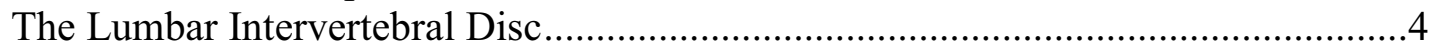

Other Components of the Human Lumbar Spine ………........................................5

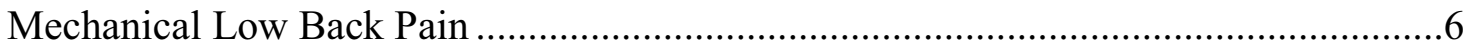

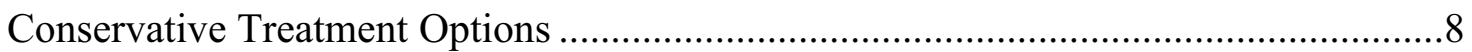

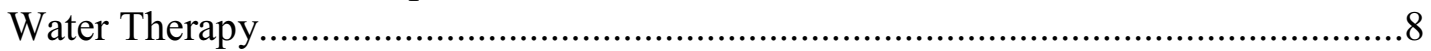

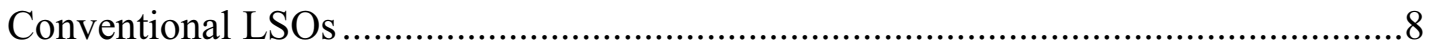



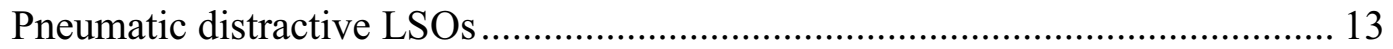

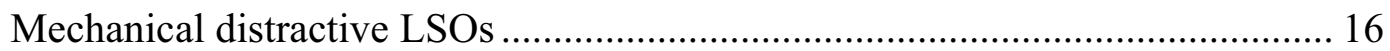

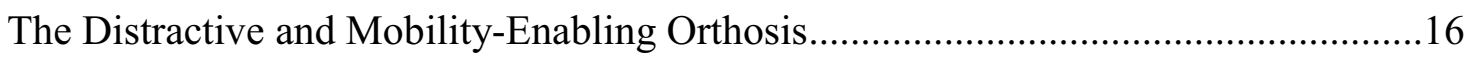

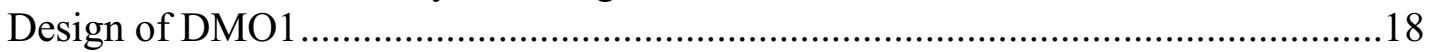

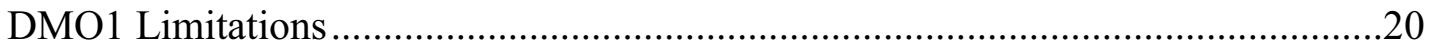

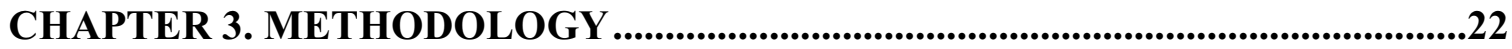

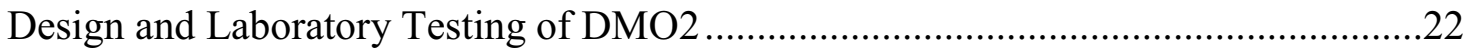

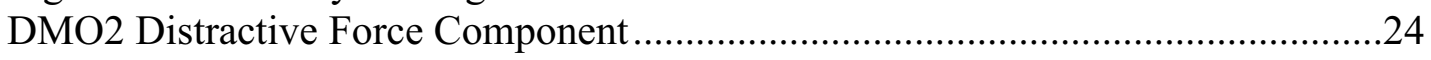

DMO2 Mobility-Enabling Component ..................................................................24

Protocol for Testing and Evaluating DMO2 in the Laboratory Under Simulated

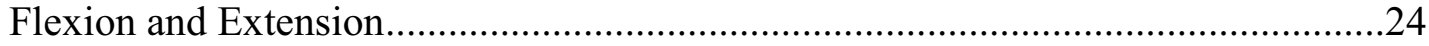

The multi-axis robotic testing platform. ……………….................................... 24

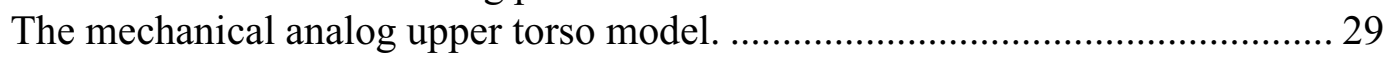

Testing protocol and force analysis. …………………....................................... 31

Design and Laboratory Testing of DMO3 …………...................................................

DMO3 Body Engagement Mechanisms ……………............................................

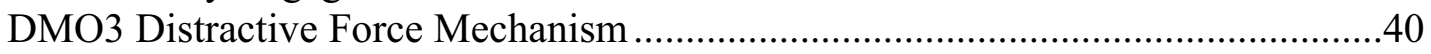

DMO3 Distractive Force Component ………………….....................................4

DMO3 Mobility-Enabling Component ...................................................................50

Protocol for Testing and Evaluating DMO3 in the Laboratory Under Simulated

Flexion and Extension......................................................................................50

Protocol for Testing and Evaluating DMO3 in the Laboratory Under Simulated

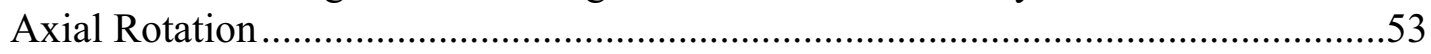

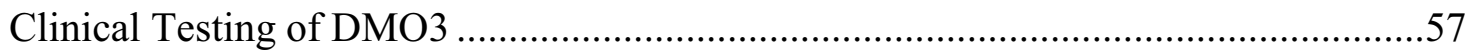

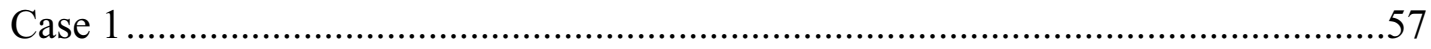

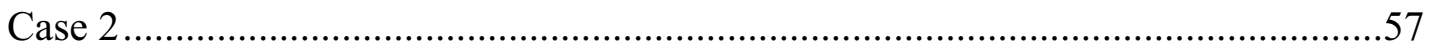

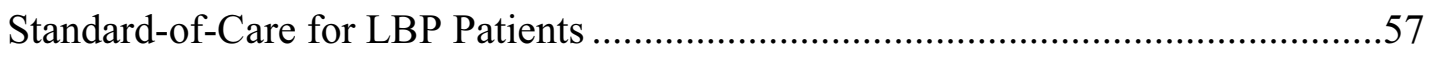

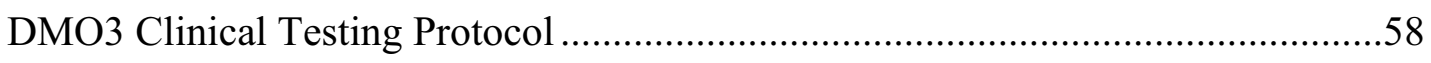

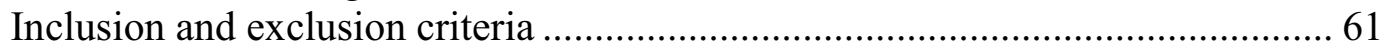

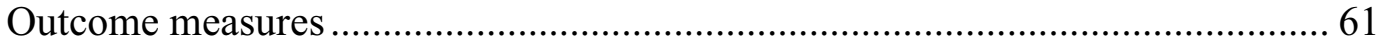




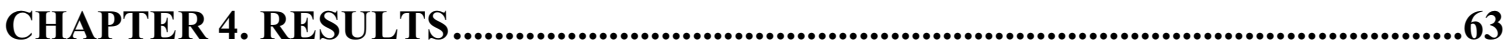

Laboratory Testing of DMO2 and DMO3 Under Simulated Flexion and Extension....63

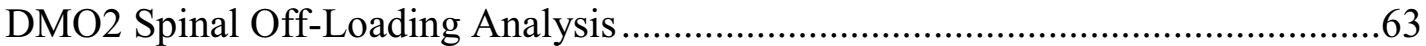

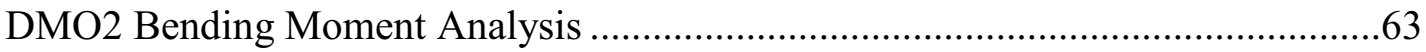

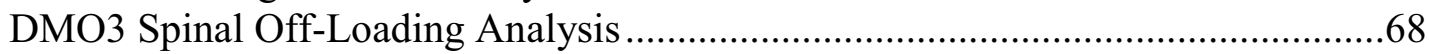

DMO3 Bending Moment Analysis .......................................................................68

Laboratory Testing of DMO3 Under Simulated Axial Rotation ..................................68

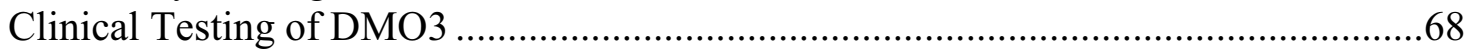

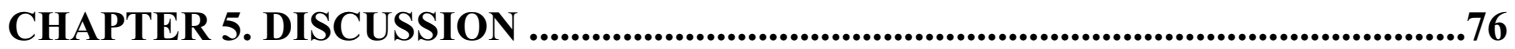

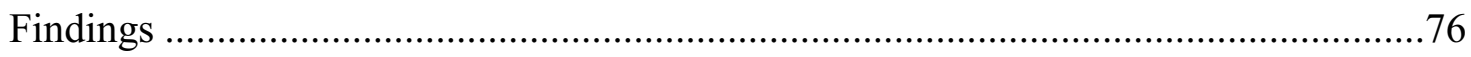

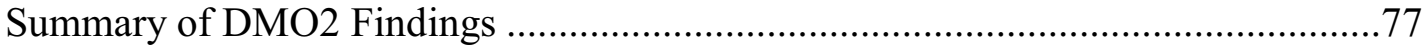

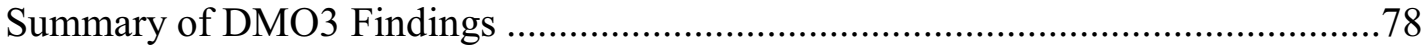

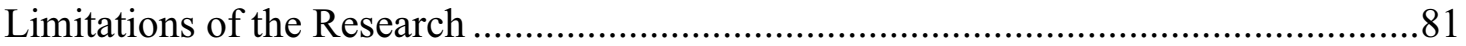

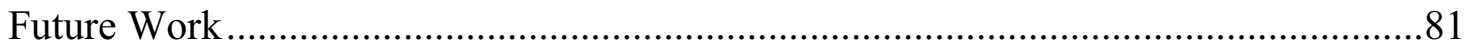

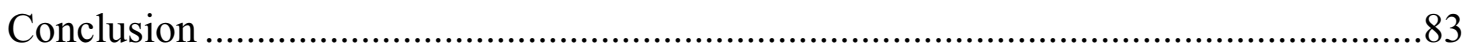

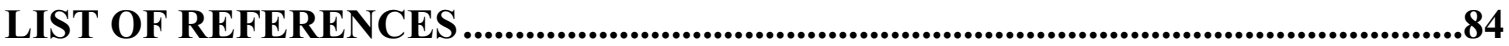

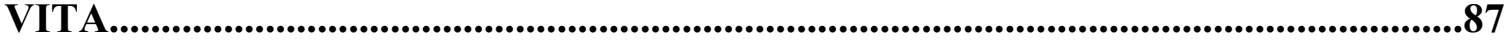




\section{LIST OF TABLES}

Table 2-1. Classification of spinal orthoses and their effects on the wearer..................9

Table 2-2. Current capacities of spinal orthoses for treating spinal diseases .................14

Table 4-1. Comparison of DMO1 and DMO2 compressive load values at upright stance and at end ranges of motion ..................................................65

Table 4-2. Comparison of DMO1 and DMO2 bending moment values at end ranges

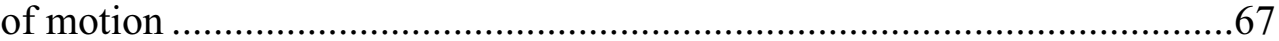

Table 4-3. DMO3 compressive load values at upright stance and at end ranges of

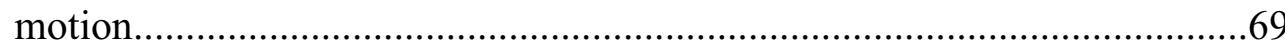

Table 4-4. DMO3 bending moment values at end ranges of motion ..........................70

Table 4-5. DMO3 axial rotation degree values for each direction of rotary turntable rotation 


\section{LIST OF FIGURES}

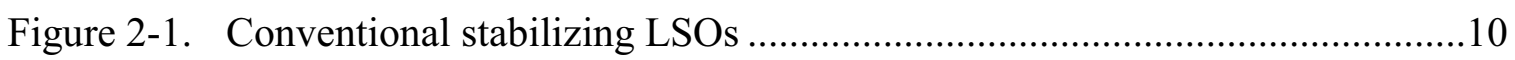

Figure 2-2. The Ex-MS1 Orthosis ……………………….......................................12

Figure 2-3. Pneumatic LSOs that provide a distractive force to the wearer by increasing pressure in pneumatic cylinders..................................................15

Figure 2-4. Mechanical LSOs that provide a distractive force to the wearer by the use of mechanical cranking mechanisms

Figure 2-5. The original DMO1 prototype

Figure 2-6. The preferred and actual behavior of the DMO1 MEC with a distractive force being applied

Figure 3-1. Flowchart of the design and evaluation process for the DMO

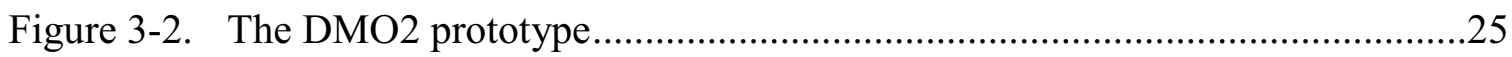

Figure 3-3. Operation of the distractive force component on DMO2 ……....................26

Figure 3-4. Operation of the mobility-enabling component on DMO2 …………….......27

Figure 3-5. Advanced testing assembly ....................................................................28

Figure 3-6. Proper placement of the rotational axis of the DMO relative to the



Figure 3-7. Advanced control strategy for minimizing off-axis force contribution to the lumbar spine at each incremental rotation...............................................32

Figure 3-8. Force analysis of the DMO2 prototype in the RTP ……………….............33

Figure 3-9. A measure of the functional ROM of the lumbar spine in flexion, extension, and axial rotation of the $\mathrm{DMO} 3$ prototype....................................35







Figure 3-13. The DMO3 pelvic belt ………………................................................... 
Figure 3-14. The four components of the distractive force component of DMO3 were the torso belt assembly, two mechanical actuators, the pelvic belt assembly, and a cable tensioning system

Figure 3-15. The torso belt assembly attaches to the torso belt by use of two Velcro straps and metal loops

Figure 3-16. The components of the torso belt assembly

Figure 3-17. The components of each mechanical actuator

Figure 3-18. The mechanical actuator guides vertical translation of two aluminum rods

Figure 3-19. The pelvic belt assembly attaches to the pelvic belt similarly to the torso belt assembly's attachment to the torso glove.

Figure 3-20. The components of the pelvic belt assembly

Figure 3-21. The pelvic belt assembly allows for flexion or extension, axial rotation, and vertical rotational axis adjustment

Figure 3-22. The distractive force component provides a distractive force to the wearer in the loaded state

Figure 3-23. The distractive force component in upright stance and axial rotation

Figure 3-24. The distractive force component in upright stance and in flexion or extension.

Figure 3-25. Set-up for testing axial rotation capacity of DMO3 with the human analog torso model mounted on a rotary turntable in the RTP

Figure 3-26. DMO3 on the human analog torso model in the RTP for axial rotation capacity testing.

Figure 3-27. Measurement method for determining axial rotation capacity of DMO3.....56

Figure 3-28. A summary of the clinical protocol for testing DMO3 on human subjects at each visit.....

Figure 3-29. X-ray measurement technique for clinical testing of DMO3

Figure 4-1. Spinal off-loading of DMO2 (bottom) compared to DMO1 (top) in upright stance and throughout extended ranges of flexion and extension ...64

Figure 4-2. Resistance to bending of DMO2 (bottom) compared to DMO1 (top) in upright stance and throughout extended ranges of flexion and extension ...66 
Figure 4-3. Spinal offloading of DMO3 in upright stance and throughout extended ranges of flexion and extension

Figure 4-4. Resistance to bending of DMO3 in upright stance and throughout extended ranges of flexion and extension.....

Figure 4-5. NPRS scores for patient 1 before and after wearing the DMO during visits 2 through 7

Figure 4-6. NPRS scores for patient 2 before and after wearing the DMO during visits 2 through 5

Figure 4-7. Modified Oswestry Disability Questionnaire scores for patient 1 between visit 2 and visit 7

Figure 4-8. Modified Oswestry Disability Questionnaire scores for patient 2 between visit 2 and visit 5

Figure 4-9. Lumbar disc height measurements for patient 1 between visit 1 and visit 7.

Figure 4-10. Lumbar disc height measurements for patient 2 between visit 1 and visit 5 


\section{LIST OF ABBREVIATIONS}

\begin{tabular}{|c|c|}
\hline $\mathrm{AF}$ & Annulus Fibrosus \\
\hline BLC & Base Load Cell \\
\hline CoR & Center of Rotation \\
\hline CTS & Cable Tensioning System \\
\hline DDD & Degenerative Disc Disease \\
\hline DLA & Daily Living Activities \\
\hline $\mathrm{DFC}$ & Distractive Force Component \\
\hline DMO & Distractive Mobility-Enabling Orthosis \\
\hline LBP & Low Back Pain \\
\hline LSCS & Lumbar Spine Core Strengthening \\
\hline LSO & Lumbar Spinal Orthosis \\
\hline MEC & Mobility-Enabling Component \\
\hline mODI & Modified Oswestry Disability Index \\
\hline NP & Nucleus Pulposus \\
\hline NPRS & Numeric Pain Rating Scale \\
\hline PT & Physical Therapy \\
\hline ROM & Range of Motion \\
\hline RTP & Robotic Testing Platform \\
\hline SDP & Sacral Disc Plane \\
\hline ULC & Upper Load Cell \\
\hline
\end{tabular}




\section{CHAPTER 1. INTRODUCTION*}

Low back pain (LBP) affects $60 \%$ to $90 \%$ of individuals at some point in their life [1]. As many as 5.4 million Americans are disabled annually as a result of LBP $[2,3]$. Back related problems remain the most expensive cause of work-related disability in terms of worker's compensation and medical expenses [4-6]. Unfortunately, LBP does not only affect the elderly; it is the most common cause of disability for those under 45 years of age [7]. The Spine Patient Outcomes Research Trial (SPORT) was designed to improve clinical decision making for surgical treatment of LBP problems. SPORT reported that approximately $70 \%$ of spinal diseases that caused LBP were mechanical in nature and included intervertebral disc herniation, spinal stenosis, and degenerative spondylolisthesis [8]. Deyo et al. reported similar findings for causative pathologies of lower back pain with $97 \%$ having a mechanical basis [6]. Although surgery is sometimes required in severe cases, conservative treatment options like PT and medication are available for those suffering from mechanical LBP. Other conservative LBP treatment methods seek to provide a distractive load to the lumbar spine to produce spinal decompression such as water therapy or use of a decompressive LSO [9-12]. Clinical studies have suggested that with adequate frequency and duration of treatment, therapeutic exercises that decompress the spine and allow movement, such as water therapy, can be beneficial in the treatment of low back pain [9-11].

LSOs have also been used to treat other spinal diseases where the role of the brace is to replace the lost mechanical function brought on by the disease and provide varying amounts and combinations of immobilization, support-stabilization, or spinal decompression [13, 14]. More recently, dynamic LSOs have been developed to provide relief from pinched nerves or disc or spinal cord compression $[12,15]$. These devices claim to axially decompress the spine but lack clinical or experimental evidence to support their efficacy. Although many different orthoses exist for treating lower back problems, we are not aware of any that provide the benefits of therapeutic exercise or enable independent living and return to active work. Such a device would well serve individuals suffering from disc degeneration, recovering from an injury, limited by weakness, and the elderly with several degenerative conditions.

The initial goal of this work was to design a back orthosis that offered spinal decompression while enabling some mobility to allow the user to engage in many daily living activities. A first prototype of a distractive mobility-enabling orthosis (DMO1) [16] was previously-designed and tested under simulated biomechanical conditions that determined the amount of spinal off-loading provided by the orthosis during vertical upright stance, initiation of flexion or extension, and over extended ranges of flexion and tested against an existing decompressive stabilizing brace and demonstrated comparable

\footnotetext{
* Portions reprinted with permission from D. DiAngelo and D. Hillyard, "A novel distractive and mobility-enabling lumbar spinal orthosis," Journal of Rehabilitation and Assistive Technologies Engineering, vol. 3, pp. 1-10, 2016.
} 
extension representative of many daily living activities [17]. The DMO1 was further spinal offloading capacity, but was unable to sustain the spinal off-loading during extended ranges of flexion or extension and became more difficult to move in at extended ranges of flexion and extension and provided no axial (twisting) movement [18].

The research motivation was to offer a conservative treatment solution for mechanical LBP by providing spinal distraction and mobility. In order to do so, one needed to overcome the design limitations of the original DMO1 prototype by redesigning the DMO in order to sustaining spinal off-loading throughout extended ranges of flexion and extension with minimal buildup of the sagittal bending moment. Collectively, the new design should be biomechanically tested under physiological daily living load and movement conditions as well as clinically tested so that it can alleviate mechanical LBP.

Our immediate goals were the following:

a) Redesign the existing DMO1 prototype and develop a new DMO prototype that sustained spinal off-loading throughout extended ranges of motion with minimal additional effort required to move

b) Test the new DMO prototype under simulated DLAs utilizing an existing laboratory testing protocol

c) Test the new DMO prototype on human subjects while performing DLAs in a controlled, clinical environment

These goals were intended to be accomplished by the following objectives organized into two phases. Phase one objectives included:

a) Decouple features of DMO1 by designing a distractive force component and a mobility-enabling component

b) Assemble a prototype (DMO2)

c) Test the functionality of DMO2 in the robotic testing platform (RTP) under simulated flexion and extension

Phase two objectives included:

a) Design engagement features of $\mathrm{DMO} 2$

b) Evaluate DMO2 and design an improved prototype (DMO3) that includes flexion, extension, and axial rotation movements

c) Test the functionality of DMO3 in the laboratory under simulated flexion, extension, and axial rotation 
d) Complete a pilot study with patients suffering from mechanical LBP using the DMO3 prototype 


\section{CHAPTER 2. BACKGROUND*}

\section{The Human Lumbar Spine}

The lumbar spine is the section of the spine in the lower back region. The lumbar spine is responsible for bearing the most weight of any part of the spine. It consists of five vertebrae (L1-L5) separated by intervertebral discs. Below the L5 vertebrae is the lumbosacral joint, also known as L5-S1, which connected the inferior lumbar spine to the superior sacrum. Unfortunately, the high loads complex movements that the lumbar spine experiences during daily living activities (DLAs) can cause pain in many individuals. Relevant anatomy and that lead to mechanical LBP will be discussed further in the following sections.

\section{The Lumbar Intervertebral Disc}

The intervertebral discs essentially act like cushions between the lumbar vertebrae. This cushion-like behavior of the intervertebral discs provides space between the lumbar vertebrae while also allowing bending, twisting and sliding movements due to disc pliancy. Each lumbar disc is approximately $10 \mathrm{~mm}$ in height; therefore, the five lumbar intervertebral discs collectively add about $5 \mathrm{~cm}$ to the length of the lumbar spine. Even though the disc must be flexible, it must also be strong and somewhat stiff in order to support and sustain mostly compressive loads between lumbar vertebral bodies. During everyday activities, water is essentially squeezed out of the lumbar discs when the individual is standing upright resulting in gradual height loss of each individual disc. This gradual height loss is a process known as creep. When the individual lies down in the recumbent position, water is reabsorbed by the disc restoring the loss of disc height. The three main components that make up the intervertebral discs are the annulus fibrosus (AF), the nucleus pulposus (NP) and the superior and inferior vertebral endplates [19].

The AF consists of about ten to twenty sheets of collagen, also known as lamellae, tightly packed in a circumferential manner around the NP. The collagen content in the AF consists primarily of both type I and type II collagen. Type I collagen content is greater towards the periphery of the AF while type II collagen is greater near the center of the disc in the AF. Because of the greater type I collagen content in the periphery of the AF, the outer $\mathrm{AF}$ tends to have a greater tensile role in disc. Within each lamellae of the AF, the collagen fibers are arranged in a parallel manner at an angle of about $65^{\circ}$ to the sagittal plane. In successive lamellae, the fibers are arranged in an opposite orientation with one layer inclined to the left and the other inclined to the right. The NP is a hydrated gel the can be found surrounded by the AF in the center of the intervertebral disc. This

\footnotetext{
* Portions reprinted with permission from D. DiAngelo and D. Hillyard, "A novel distractive and mobility-enabling lumbar spinal orthosis," Journal of Rehabilitation and Assistive Technologies Engineering, vol. 3, pp. 1-10, 2016.
} 
gel expands radially under compression which is resisted by the AF. The AF is supported on the inside by the NP which prevents the AF from buckling inwards and losing its stiffness. Therefore, both the AF and NP function to provide stiffness to the disc under compressive loading while still maintaining compliancy needed for movement between vertebral bodies. Consisting mostly of proteoglycans, the NP is the primary water absorbing component of the intervertebral disc and displays many ideal, hydrodynamic properties. The vertebral endplates are cartilaginous plates that bind the disc to their respective vertebral bodies. It covers almost the entire surface of the adjacent vertebral body with only a narrow portion of bone, called the ring apophysis, around the outside of the vertebral body left uncovered [19].

Lumbar intervertebral discs receive nervous system input and nutrients from a variety of sources. The AF receives nerves from many sympathetic trunk-derived fine nerves that covers the vertebral column anteriorly and laterally. Posteriorly, the AF receives sympathetic input from a plexus that covers the floor of the vertebral canal. Many of the nerve endings in the AF are free nerve endings [19]. A study in 1997 showed nerve endings were present in the subchondral bone of the vertebral endplates of lumbar discs, but their function is not known [20]. The intervertebral disc of the lumbar spine receive a relatively poor blood supply limited to tiny vessels that are extensions of external arteries that supply adjacent vertebral bodies. Because of this, the discs rely heavily on diffusion, primarily from vessels around the periphery of the annulus, for their nutrition. Nutrients such as glucose and oxygen are able to go down their respective concentration gradients to enter this disc; however, their diffusion is slow because of the density of proteoglycans in the disc. Movement aids nutrition of the disc because movement causes bulk flow of water and nutrients into and out of the disc [19]. Primarily, diffusion of nutrients into the disc occurs either from the marrow of the vertebral bodies across the subchondral bone and cartilaginous end plate or through the AF from the surrounding blood vessels [21]. These nutrients that flow into and out of healthy spinal discs allow the disc height to be maintained at a healthy height.

\section{Other Components of the Human Lumbar Spine}

Some other important components that make up the lumbar spine in addition to the lumbar intervertebral disc include vertebral bodies, posterior elements, ligaments, zygapophysial joints, the sacrum and spinal nerves. The vertebral bodies support about half of the weight of your body with the other half likely supported by muscles. Some of the important posterior elements include the pedicles, the transverse and spinous processes and the lamina. While many of the structures in the spine are called ligaments, they are not truly ligaments either because they do not connect two bones or they are too feeble to serve as ligaments. The most definitive ligament, made of elastin, is the ligamentum flavum which is very extensible when the lumbar vertebral column bends forward. The zygapophysial joints provide an important mechanism between consecutive lumbar vertebrae as they are designed to block axial rotation and forward sliding of the lumbar vertebrae. The sacrum has two main functions including supporting the lumbar vertebral column and transmitting forces between the vertebral column and the lower 
limbs. Each spinal nerve, located in the intervertebral foramina, is connected to the spinal cord by a dorsal root and a ventral root. Each is a mixed nerve containing both sensory and motor fibers [19].

\section{Mechanical Low Back Pain}

LBP is the leading cause of activity limitation and work absence throughout much of the world. Furthermore, LBP causes more global disability than any other condition [22]. Unfortunately, no clinically identifiable diagnosis can usually be confirmed as the source of the symptoms of LBP experienced by the patient. Risk factors include, but are not limited to, genetic risk factors associated with specific genes inherited from parents, individual risk factors such as body height and weight, environmental risk factors such as occupation and sporting activities, and psychosocial risk factors such as depressive mood and somatization. Adolescents seem to have prevalence rates similar to those for adults, although the pain is readily forgotten and disability is rare [19].

Symptoms may be different depending on the cause of LBP. Sciatica, which is the presence of leg pain with LBP, is strongly associated with herniated lumbar disc, peaking between the ages of 40 and 45 years [19]. Pain that is present when sitting but relieved when the patient is lying supine is a common complaint of a patient with a disc problem. Most patients and therapists assume exercise will make backs stronger and eliminate symptoms, but there is no strong evidence that strong muscles alone will protect the back from painful episodes [23]. Back pain is conventionally described as acute (less than 6 weeks), subacute (6 weeks to 3 months) or chronic (longer than 3 months) [24]. The best single predictor of future back pain is a previous history of back pain which outweighs any other predictor [19].

LBP diagnosis can either be mechanical, non-mechanical, or visceral with approximately $97 \%$ of cases being mechanical in nature. Imaging can be helpful in diagnosis and determination of clinical treatment methods. Most commonly, computed tomography (CT) and MRI are used as they are more sensitive than plain radiography for viewing soft tissue and detecting early spinal injury or diseased conditions. Also, these imaging techniques can reveal mechanical conditions such as herniated disks and spinal stenosis while plain radiography cannot [6].

Common outcomes, or measureable parameters, that could determine success of a clinical trial that seeks to relieve patients of mechanical LBP are pain and disc height. These parameters are not the only possible parameters; however, they may be the best choices for those suffering from a LBP disease that is mechanical in nature. LBP is a big problem that has yet to be solved for all cases. For this reason, there is an urgent need for further research to better understand LBP and the best way to reduce pain in those who suffer from mechanical LBP [22].

Degenerative disc disease (DDD) and diseases that stem from DDD, such as spinal stenosis, remain among the leading causes of functional disability in both males 
and females and often causes people to have to take extended periods of time off from work. People who suffer from DDD can go undetected for long periods of time until they go to the doctor and are diagnosed, likely with the assistance of imaging. The source of DDD can be a variety of things from genetic influences to impaired nutrient delivery to the disc. Imaging can be an invaluable tool in helping to detect and influence therapeutic decision making for those suffering from DDD. Although degeneration can occur at any age, it is more common in older discs [19]. By the age of 50 years, $85 \%$ to $95 \%$ of adults show evidence of DDD at autopsy [21].

Disc degeneration involves both disruption in structural integrity and cellmediated changes in compositions; however, it is unclear which happens first. Degeneration involves structural changes which are most evident in the AF and vertebral end plates. With degeneration and aging, type II collagen increases radially in the AF and there is a greater water loss from the NP than from the AF [19]. Reduced blood flow in the vertebral end plate initiates breakdown first in the endplate and then in the nucleus. These changes can alter proteoglycan integrity in the NP reducing the movement of solutes into and out of the disc [21]. Any cause or disease that lessens the integrity of proteoglycans in the NP lessens the ability of the disc to restore and maintain height [19]. The NP, with loss of water and proteoglycan integrity, becomes a yellow-brown color and the appearance begins to unravel revealing cracks, clefts or crevices that sometimes extend into the AF [21]. Dehydration of the NP affects the behavior of the disc and its response to loading which can ultimately lead to a breakdown in the AF and vertebral end plates. This breakdown limits the ability of the disc to resist loading effects during normal daily living activities (DLAs). Because of this, DLAs can sometimes accelerate further damage to the disc. Mechanical overloading of the disc can sensitize painsensitive nerves making one feel pain more often [15]. Fissuring, chondrocyte generation and granulation tissue formation may be present within the endplate, AF, and NP of degenerated discs indicating attempts at healing. Imaging can help to detect decreased disc height, degeneration of ligaments and facet joints, or even decrease in signal intensity of adjacent vertebral bodies indicating changes to the composition of the bone in the vertebral bodies [21].

Common potential complications of DDD include abnormalities in lumbar alignment, displacement in disc space, and spinal stenosis. Three types of disc prolapse are disc protrusion, disc extrusion, and sequestration which is often categorized by the amount that intervertebral disc contents are intact with adjacent structures. Spinal stenosis occurs from degenerative facet joints which often leads to intrusion of spinal nerves resulting in pain [19].

The most common symptom for DDD is pain. This disease can be causes by a combination of factors including instability with associated disc degeneration, facet hypertrophy or arthopathy, mechanical compression of nerves by bone, ligament, or disc material, and biochemical mediators of inflammation and pain. A combination of these factors often leads to mechanical compression or deformity of nerve roots which often results in pain or nerve dysfunction [21]. The most common surgical treatment option for those with DDD is spinal fusion which seeks to limit motion at the diseased lumbar level. 


\section{Conservative Treatment Options}

Treatment methods for those suffering from mechanical LBP vary from rest to surgery. Many conservative treatment options for mechanical LBP such as physical therapy (PT), traction, and bracing can be effective in reducing short-term pain depending on the severity of the disease and the dedication to treatment [25]. For example, supine decompression by a motorized decompression system such as the DRX9000. has been proven to restore disc height while reducing pain in patients with LBP [26]. Other studies have concluded that bracing can be an effective treatment option for reducing short-term LBP and improving short-term function in people with chronic LBP [25]. However, there are other conflicting reviews as to whether nonsurgical methods can produce the same positive effects as surgical methods in individuals suffering from LBP [27, 28]. Most conservative treatment options for those with mechanical LBP aim to strengthen core stability and develop motor control to improve muscle coordination; however, those with mechanical LBP have pain as his or her limiting factor. Two common conservative treatment options that have been effective in treating mechanical LBP are water therapy and bracing with a LSO.

\section{Water Therapy}

Water therapy has proven successful for those suffering from LBP $[9,10]$. The believed mechanism by which water therapy reduces LBP is the buoyancy force provided by the water reduces the effects of one's torso weight. This apparent reduction in torso weight results in a reduced pressure on the lumbar discs which in itself is likely to provide some pain relief. The combination of water therapy and exercise has been proven to be beneficial. This combination could encourage nutrient bulk flow into the disc and result in an increase in core muscle strength. Studies have concluded that "with adequate frequency and duration of treatment, water therapy can be beneficial in the treatment of LBP" [9]. One issue with water therapy is that patients are not able to use the pool whenever he or she wants to seek the distractive benefits that are associated it. Unfortunately, it can sometimes be inconvenient for a person to have to go to a special facility or location just to use the pool to seek relief from mechanical LBP.

\section{Conventional LSOs}

Many conventional LSOs attempt to provide a force to the wearer in order to improve a spinal disease or condition. Particularly in the case of low back pain, the method by which spinal orthoses are prescribed to patients is random and empirical. Table 2-1 provides a classification of orthoses that will be discussed. By classifying LSOs according to their effects on the wearer, clinical outcomes such as reducing low back pain can be improved more frequently.

Most conventional LSOs seek to immobilize or stabilize the wearer's lumbar spine. The Aspen LSO (Aspen Medical Products, CA, USA), as shown in Figure 2-1a, 
Table 2-1. Classification of spinal orthoses and their effects on the wearer

\begin{tabular}{ll}
\hline \multicolumn{1}{c}{ Orthosis Classification } & \multicolumn{1}{c}{ Effects on the Wearer } \\
\hline Distractive force & $\begin{array}{l}\text { Attempts to decompress (increase disc } \\
\text { height) the lumbar spine by offloading } \\
\text { spinal load and transferring it to the pelvic } \\
\text { girdle }\end{array}$ \\
Immobilizing & $\begin{array}{l}\text { Almost completely eliminates mobility in } \\
\text { any direction }\end{array}$ \\
Applies a force to a defective spinal \\
curvature such as scoliosis \\
Reduces or eliminates mobility in at least \\
Stabilizing & one motion plane \\
\hline
\end{tabular}




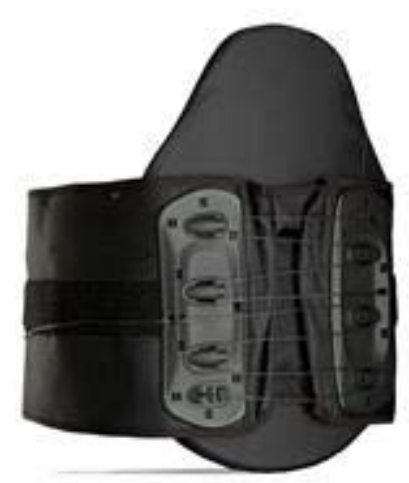

(a)

(c)

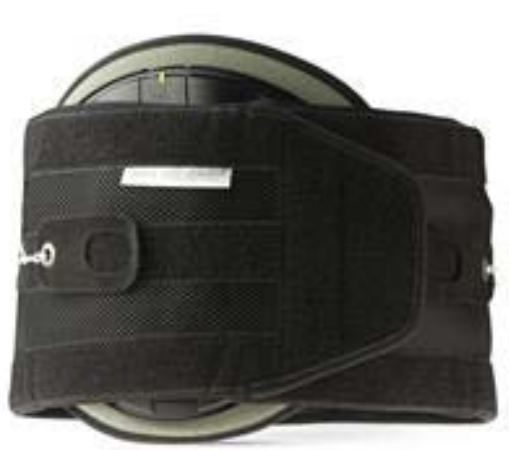

(b)

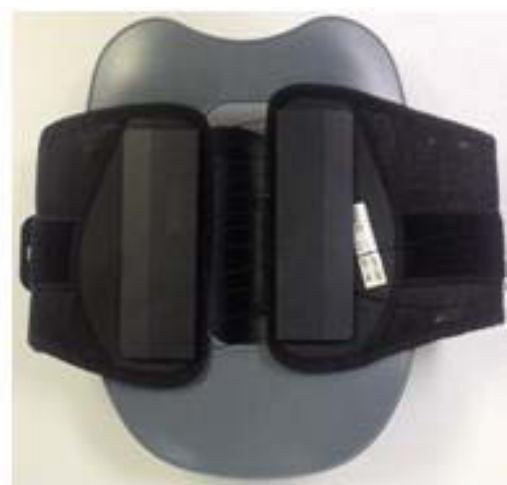

Figure 2-1. Conventional stabilizing LSOs

(a) The Aspen LSO (Aspen Medical Products, CA, USA)

(b) The Cybertech 2000 Orthosis (Biocybernetics International, CA, USA)

(c) The QuikDraw Brace (Aspen Medical Products, CA, USA) 
attempts to increase abdominal pressure and thereby stabilizing one's upper torso while simultaneously correcting lumbar lordosis. Lumbar lordosis can be adjusted in this orthosis by bending the lumbar support rods so that the desired lumbar lordosis can be produced. The Cybertech 2000 orthosis (Biocybernetics International, CA, USA), as shown in Figure 2-1b, is another stabilizing orthosis that corrects lumbar lordosis. Like the Aspen LSO, the Cybertech 2000 orthosis increases abdominal pressure through a pulley system located in the posterior portion of the orthosis. The wearer will pull on the pulley tabs that are located on the wearer's lateral sides of the orthosis until the desired amount of abdominal pressure is reached. Unlike the Aspen LSO, the Cybertech 2000 orthosis corrects lumbar lordosis through different lumbar pad inserts that can be inserted into the orthosis. The QuikDraw Brace (Aspen Medical Products, CA, USA), which can be seen in Figure 2-1c, stabilizes the wearer's trunk like the previous orthoses described; however, this orthosis does not an attempt to correct lumbar lordosis. One study prescribed the QuikDraw Brace to patients suffering from acute low back pain for two weeks in order to analyze patient quality of life as measured by the modified Oswestry Diability Index (mODI) and spinal function as measured by a timed test of lumbar extension against gravity. The study concluded that the orthosis either improved spinal function or did not worsen spinal function. Also, quality of life was improved by prescription of the stabilizing orthosis [29].

One company has developed an electromechanically-activated orthosis called ExMS 1 (Exo Dynamics, LLC, MI, USA) as shown in Figure 2-2a. Unlike conventional stabilizing LSOs, this orthosis does not see seek to increase abdominal pressure of the wear. It seeks to reduce muscle effort of the wearer by transferring some of the wearer's torso weight to the wearer's hips. Essentially, the orthosis provides a corrective moment to the wearer's trunk as he or she attempts to bend forward in flexion or backward in extension to reduce the postural muscle effort required to maintain his or her posture. The company claims that by reducing the muscle effort required to bend forward in flexion and extension, the corresponding compressive forces on the wearer's spinal column are reduced. An illustration of this orthosis providing a corrective moment to a wearer in forward flexion, termed "motion following" technology, can be seen in Figure 2-2b. Another view of the same orthosis in forward flexion can be seen in Figure 2-2c. The actuators are controlled by pressure sensors that are connected to air bladders located on each side of the midline at the xiphoid process. Each pressure sensor is programmed to have a minimum pressure and maximum pressure threshold. When one attempts to bend forward in flexion, pressure read by the pressure sensor increases above the maximum threshold which causes the anterior actuators to retract and the posterior actuators extend. If one attempts to bend backward in extension, pressure read by the pressure sensor decreases below the minimum threshold which causes the anterior actuators to extend and the posterior actuators to retract [30]. This orthosis can be classified as a stabilizing orthosis. Because it does not apply a distractive force across the wearer's lumbar spine, it is not considered a dynamic orthosis. 


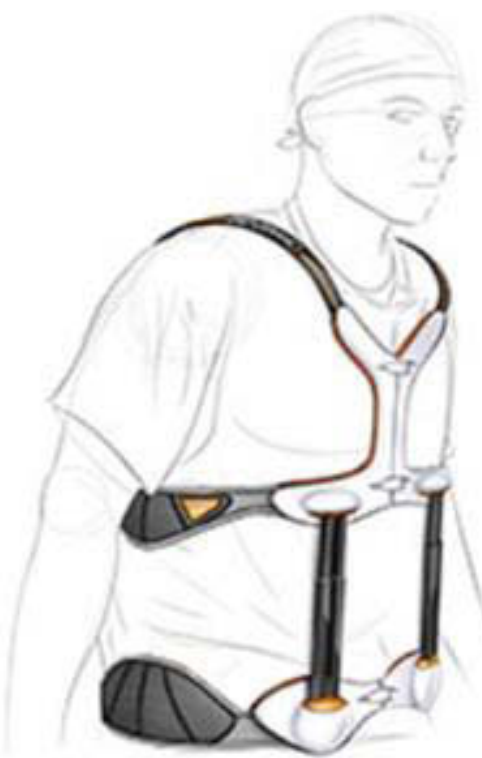

(a)

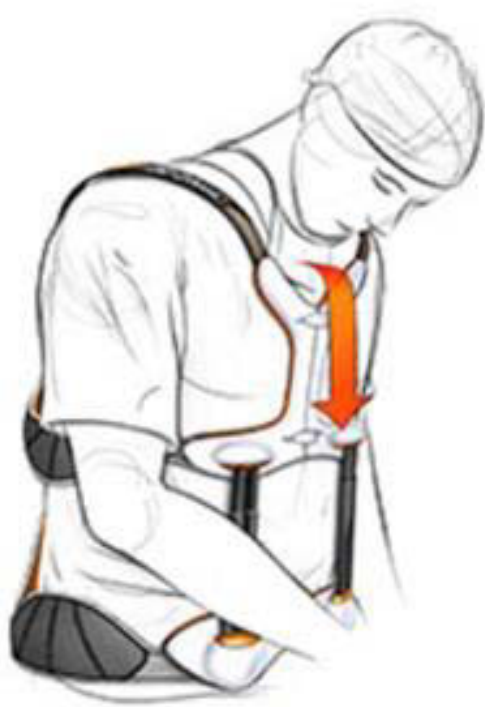

(b)



(c)

Figure 2-2. The Ex-MS1 Orthosis

(a) The Ex-MS1 orthosis in upright stance

(b) The orthosis provides a corrective moment to the wearer when undergoing forward flexion

(c) A posterior view of the wearer in forward flexion 


\section{Dynamic LSOs}

LSOs that provide a distractive force across the lumbar spine and stabilize the wearer's trunk are classified as dynamic LSOs. Other orthoses may be more useful for treating spinal diseases other than degenerative disc disease or lumbar spinal stenosis. Table 2-2 attempts to determine how spinal orthoses meet or do not meet needs associated with many common spinal diseases.

Some dynamic LSOs attempt to decrease the load on the lumbar intervertebral disc by applying a distractive force across the lumbar spine. This distractive force applied by a dynamic LSO essentially simulates the buoyant effect of water therapy without the burden of going to a special facility in order to get pain relief. The clinical rationale for dynamic LSOs decreasing pain and improving quality of life for patients with mechanical LBP is found in providing spinal stability while simultaneously decreasing the pressure on the lumbar intervertebral disc. Stabilizing the lumbar spine is thought to decrease the histological and inflammatory cascade that can, in some cases, cause mechanical LBP [15]. A review of the literature has shown that dynamic LSOs provide their distractive force across the lumbar spine either through the utilization of pneumatic cylinders or by mechanical traction.

Pneumatic distractive LSOs. The Orthotrac pneumatic vest (Orthofix, Inc., TX, USA), as shown in Figure 2-3a, provides a distractive force to the wearer by separating the upper and lower sections of the orthosis by increasing pressure in pneumatic cylinders with a pump. A set of pneumatic cylinders are located on the anterior portion of the orthosis and another set is located on the posterior portion of the orthosis. This orthosis seeks to stabilize the wearer's trunk and does not permit much flexion, extension, axial rotation, or lateral bending. One study attempted to determine if the distractive force provided by the Orthotrac could decrease L4/L5 or L5/S1 intradiscal pressure in a cadaver torso in a laboratory setting. It was ultimately determined that the orthosis was able to decrease the intradiscal pressure by approximately $25 \%$ when the pneumatic cylinders were providing approximately $400 \mathrm{~N}$ of distractive force to the cadaver torso. A distractive force of approximately $140 \mathrm{~N}$ resulted in a decrease of $18 \%$ in intradiscal pressure [15].

The DDS 500 decompression belt (Disc Disease Solutions, NJ, USA), as shown in Figure 2-3b, claims to decompress the wearer's lumbar spine by increasing abdominal pressure when air is pumped into pneumatic cylinders embedded in the orthosis and dynamically inflating simultaneously. One study attempted to measure the effects of this orthosis to restore spinal disc height after an acute bout of exercise. The subject population were students from The University of Waterloo in Ontario, Canada that had no previous history of disabling back pain. Subjects acted as their own control. All subjects would initially have their spinal height measured by a stadiometer (accuracy \pm $0.03 \mathrm{~mm}$; resolution $0.01 \mathrm{~mm}$ ), undergo an exercise session, and then have his or her spinal height measured by the stadiometer again. On the control visit, the subjects did not wear the orthosis after the second spinal height measurement, and on the experimental visit, the subjects did wear the orthosis for 20 minutes while lying supine and then had 
Table 2-2. Current capacities of spinal orthoses for treating spinal diseases

\begin{tabular}{|c|c|c|c|}
\hline Spinal Disease & Lost Mechanical Function & $\begin{array}{c}\text { Target } \\
\text { Population }\end{array}$ & $\begin{array}{l}\text { Met/Unmet } \\
\text { Needs }\end{array}$ \\
\hline Fusion surgery & Mobility & $\begin{array}{l}\text { Injury } \\
\text { Elderly }\end{array}$ & $\begin{array}{l}\text { Treated w/ } \\
\text { stabilizing brace }\end{array}$ \\
\hline $\begin{array}{l}\text { Spondylolisthesis/ } \\
\text { Spinal stenosis }\end{array}$ & $\begin{array}{l}\text { Translational stability } \\
\text { Axial stability and support } \\
\text { Rotational stability } \\
\text { Positional integrity }\end{array}$ & Elderly & $\begin{array}{l}\text { Treated w/ } \\
\text { stabilizing brace }\end{array}$ \\
\hline Scoliosis & $\begin{array}{l}\text { Positional integrity } \\
\text { Rotational and axial stability }\end{array}$ & $\begin{array}{l}\text { Typically } \\
\text { younger } \\
\text { patient }\end{array}$ & $\begin{array}{l}\text { Treated w/ } \\
\text { stabilizing and } \\
\text { shape correcting } \\
\text { brace }\end{array}$ \\
\hline $\begin{array}{l}\text { Pinched } \\
\text { nerve/Disc or } \\
\text { cord compression }\end{array}$ & $\begin{array}{l}\text { Axial stability } \\
\text { Translational stability }\end{array}$ & $\begin{array}{l}\text { Young adult } \\
\text { Elderly }\end{array}$ & $\begin{array}{l}\text { Treated w/ } \\
\text { distractive, } \\
\text { stabilizing brace } \\
\text { "UNMET } \\
\text { NEEDS" }\end{array}$ \\
\hline Weak elderly & $\begin{array}{l}\text { Translational stability } \\
\text { Axial stability and support } \\
\text { Rotational stability } \\
\text { Positional integrity }\end{array}$ & $\begin{array}{l}\text { Older } \\
\text { patients } \\
\text { with chronic } \\
\text { LBP }\end{array}$ & $\begin{array}{l}\text { Spinal } \\
\text { decompression } \\
\text { Mobility (when } \\
\text { mobility is } \\
\text { indicated) }\end{array}$ \\
\hline Disc degeneration & $\begin{array}{l}\text { Axial stability and support } \\
\text { Rotational stability }\end{array}$ & $\begin{array}{l}\text { Young often } \\
\text { w/ injury }\end{array}$ & $\begin{array}{l}\text { Spinal } \\
\text { decompression } \\
\text { Mobility }\end{array}$ \\
\hline
\end{tabular}




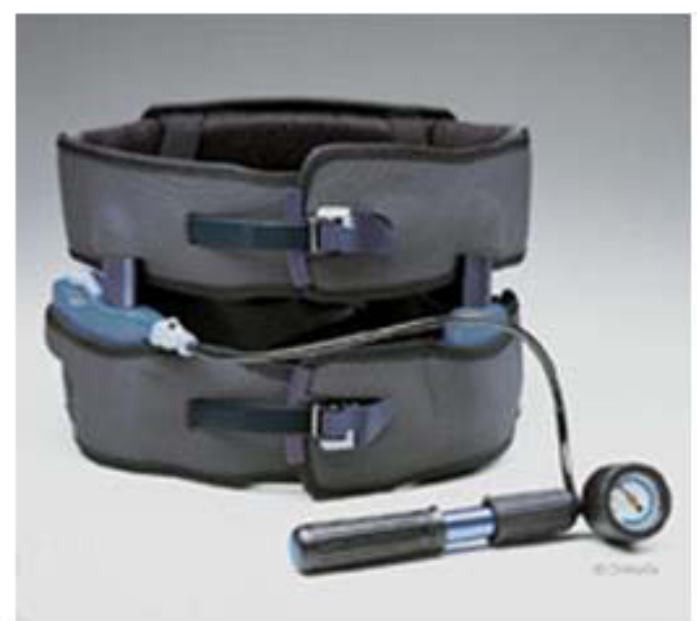

(a)

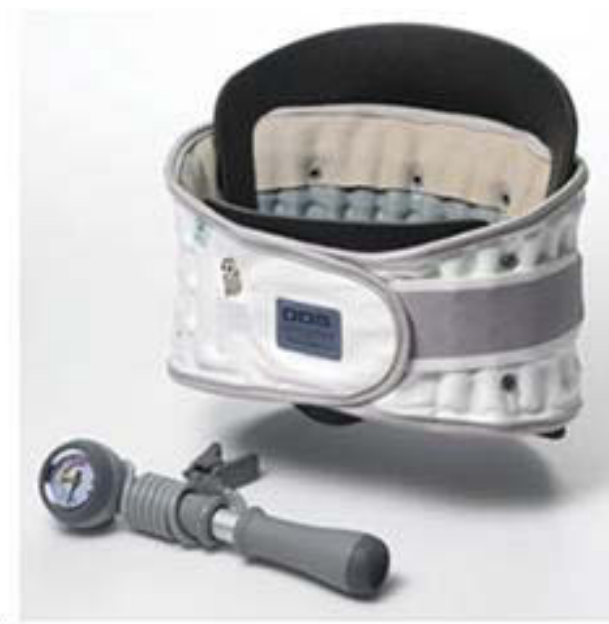

(b)

Figure 2-3. Pneumatic LSOs that provide a distractive force to the wearer by increasing pressure in pneumatic cylinders

(a) The Orthotrac Pneumatic Vest (Orthofix, Inc., TX, USA)

(b) The DDS 500 Decompression Belt (Disc Disease Solutions, NJ, USA) 
his or her spinal height remeasured for a third time. The study concluded the use of the DDS 500 decompression belt increased spinal height, as measured by the stadiometer, significantly more than the control condition following an acute bout of exercise [31].

Mechanical distractive LSOs. The Vertetrac Traction System (Meditrac Ltd, TX, USA), as shown in Figure 2-4a, is a vertical ambulatory traction device that seeks to provide a distractive force across the spine and correct lumbar lordosis in an attempt to decompress the lumbar spine. This device is marketed as "traction on the move" as the user is encouraged to wear this device for twenty to thirty minutes a day while performing DLAs. The device functions as a dynamic frame corset that applies a distractive force between the wearer's iliac crest and ribs. This distractive force is applied by two mechanical one-way cranking mechanisms that are located on the left and right sides of the posterior portion of the device. After application of the distractive force, one is able to adjust the amount of lumbar lordosis with an adjustable level arm that is located in the posterior portion of the brace and attached to the inferiorly located belt. One study attempted to examine if traction, as applied by the Vertetrac, and walking in combination are more effective than the wearing Vertetrac alone. Two groups of patients with degenerative disc disease in the lumbar spine and associated LBP that had been persistent for at least six months and no longer than two years were formed. Group 1 was treated with the Vertetrac only, and Group 2 was treated with the Vertetrac while walking. Each group underwent twenty treatment sessions within a twenty-eight days. Patients were evaluated for pain and lumbar ROM before treatment, one month, six months, and one year upon completion of the treatment protocol. While both groups experienced a statistically significant decrease in pain, the difference in pain in Group 2 was significantly greater than Group 1 at the one month, six months, and one-year follow-up examinations. A trend that was noticed in the study was that the greatest decrease in pain came between the initiation of treatment and the one-month follow-up examination. After that, gradual loss of good pain results was noticed. For both groups, the pain increased between the one month and six months follow-up visit and the six months and one year follow-up visit [12].

The VerteCore Lift (VerteCore Technologies, TX, USA) shown in Figure 2-4b provides a distractive force between the wearer's arm pits and the wearer's iliac crest. The distractive force is applied by two adjustable decompression jacks located on the left and right sides of the wearer's body. By stroking the handle connected to the decompression jack, the jack extends pushing up underneath the arm pits and down on the iliac crest. Like the Vertetrac, the VerteCore Lift is marketed in such a way that encourage activity while wearing the brace. Wearers are suggested to wear the dynamic LSO for approximately thirty minutes a day while performing normal, low-impact DLAs such as walking or doing yardwork [32].

\section{The Distractive and Mobility-Enabling Orthosis}

Some of the previously-discussed conservative treatment options may be enticing to individuals suffering from mechanical acute or subacute LBP as they are proven to 


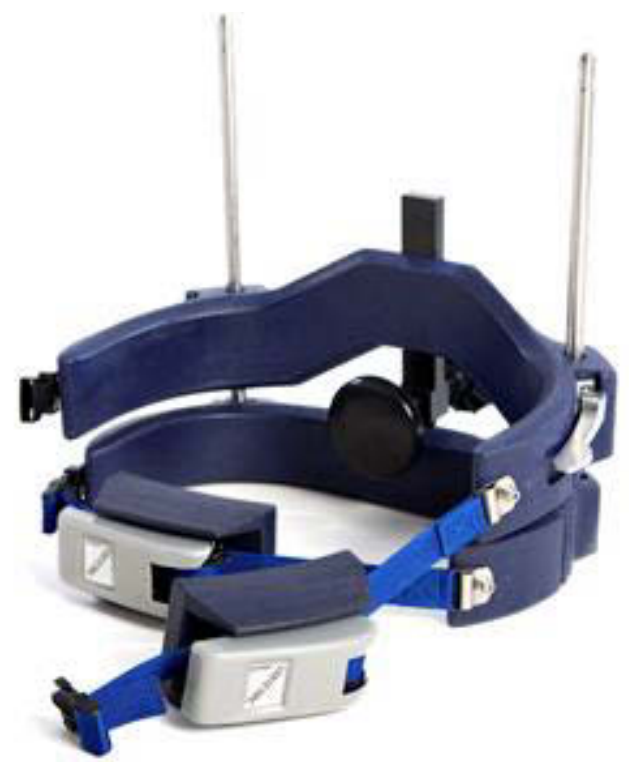

(a)

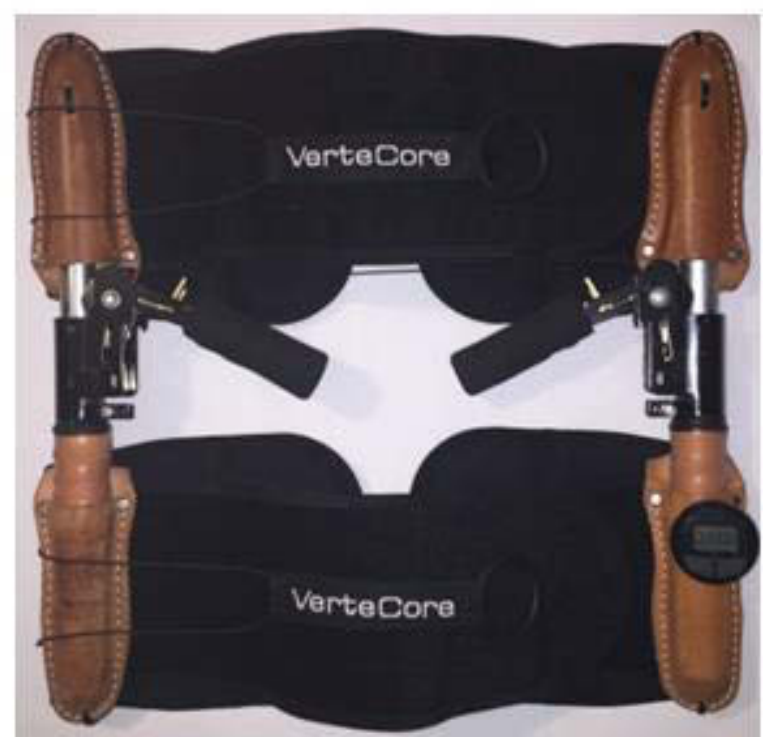

(b)

Figure 2-4. Mechanical LSOs that provide a distractive force to the wearer by the use of mechanical cranking mechanisms

(a) The Vertetrac Ambulatory Traction System (Meditrac Ltd, TX, USA)

(b) The VerteCore Lift (VerteCore Technologies, TX, USA) 
decrease pain in the short term. While all of these treatment methods are preferable to surgery, they all have limitations. Both the conventional LSOs and dynamic LSOs previously mentioned do not allow for natural flexion, extension, axial rotation of lateral bending about some point or axis of the lumbar spine. These devices restrict and prevent the wearer from bending to protect against the instability of the lumbar spine. This is not necessarily the most comfortable and natural feeling for the wearer. Also, this could lead to muscle atrophy of the back and abdominal muscles causing the wearer to become reliant upon the orthosis. Therefore, the LSO is only an effective treatment while the wearer is wearing the orthosis. Many of these LBP patients are told to exercise or perform some PT program in order to strengthen core muscles and improve stability of the lumbar spine; however, for many pain itself is a limiting factor in being able to perform these exercises effectively. Table 2-2 highlights an unserved population including the weak elderly and those suffering from DDD that could benefit from decompression and mobility, a proven conservative treatment option in reducing LBP [911]. The main advantage of a distractive and mobility-enabling orthosis (DMO) compared to water therapy or PT is that one is not reliant on special equipment and having to travel to a facility in order to experience the benefits that each type of therapy provides. While wearing the DMO, the weak muscles and likely degenerative discs in the weak elderly patients could benefit from the support of LSO. The combined ability to move with a constantly-applied distractive force would make it more likely that the weak elderly can perform DLAs such as housework and yardwork without the assistance of others. With decreased pressure on the lumbar intervertebral discs and the ability for movement, the younger DDD population could potentially encourage the bulk flow of nutrients into the disc to temporarily restore disc height and therefore improve the disc's ability to support forces and moments experienced during many DLAs. This temporary restoration in disc height would likely decrease his or her pain while the wearer is not wearing the DMO. This unserved population combined with the desired non-reliance on special equipment or travel to a facility for treatment led to the motivation to design, build, and test the first prototype of the DMO which will herein be referred to as DMO1. The goal of the DMO1 was to provide a distractive force across the wearer's lumbar spine while allowing flexion and extension motion with minimal resistance to bending.

\section{Design of DMO1}

The original DMO1 prototype is shown in Figure 2-5a and had two unique design features: a distractive force component (DFC) and a mobility enabling component. Each component was placed on the left and right lateral sides of orthosis. The DFC consisted of a cable pulley system and a flexible graphite rod that was anchored to the pelvic belt as shown in Figure 2-5b. The cable pulley system attached to the lower part of the torso glove and to a free-floating coaster. Once the pulley system was engaged, tension on the cable caused the cable pulley system to pull the coaster against flexible rod. As the flexible rod deflected under the coaster's load, the base of the torso glove was pulled up to engage the torso. A band was placed between the vertically orientated rods to control its structural bending property, acting much like a mid-support of a vertical column carrying a buckling load. The MEC, which can be seen in Figure 2-5c, consisted 


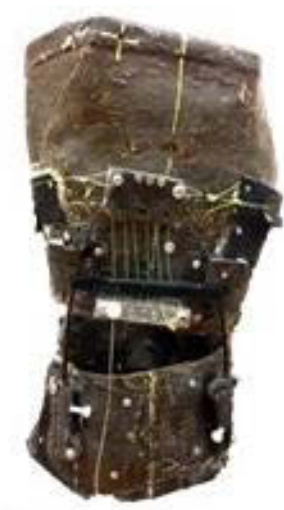

(a)

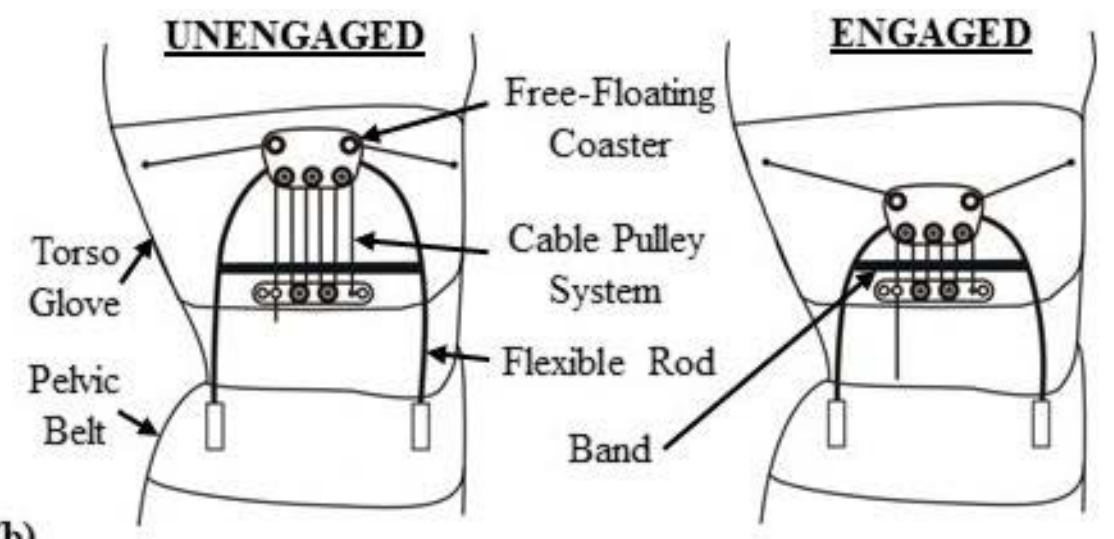

(b)

VERTICAL STANCE

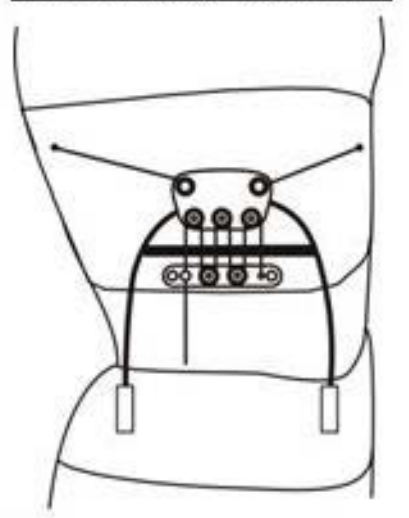

FLEXION

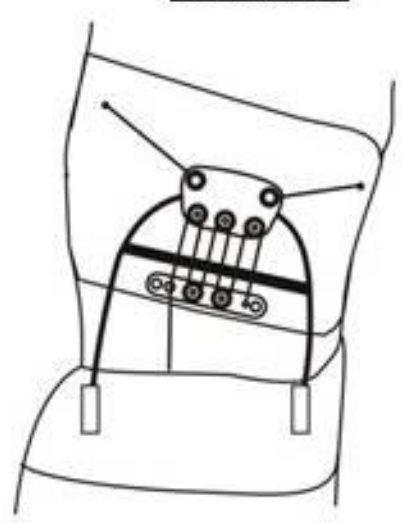

(c)

Figure 2-5. The original DMO1 prototype

(a) A photograph of the side view of the DMO1 prototype

(b) The DMO1 distractive force component in the unengaged and engaged states

(c) The DMO1 mobility-enabling component in vertical stance and forward flexion 
of the flexible graphite rod and the free-floating coaster that was tethered to the torso glove. With the pulley system engaged, the coaster was unconstrained and allowed to roll freely along the curved portion of the rod allowing flexion and extension of the torso glove relative to the pelvic belt.

\section{DMO1 Limitations}

The first prototype, DMO1, had a limitation that restricted it from accomplishing its intended goals. This limitation is illustrated in Figure 2-6. With the cable pulley system engaged and the flexible graphite rod deflected, the wearer would begin to bend forward in flexion. As the wearer flexed in forward flexion, it was preferred that the freefloating coaster remained normal to the axis that was normal to the surface of the graphite rod and that radius of curvature of the rod remained close to the rotational axis of the lumbar spine. The more that that was the case, the less additional resistance to bending the DMO1 was produced. Unfortunately, as the wearer flexed, the curvature of the rod did not remain near the lumbar spine's rotational axis. Further, the coaster did not roll freely on the rod and eventually the coaster jammed on the flexible rod after about 15 degrees of forward flexion. When this occurred, off-axis forces were generated that caused additional resistance to bending because now the wearer would have to overcome both the spinal tissue's resistance to bending as well as the off-axis rod forces providing a counter moment to the wearer. When the free-floating coaster began to jam on the rail, the flexible rod would begin to shear forward and the approximate CoR of DMO1 would no longer be closely aligned with the CoR of the lumbar spine. This design limitation ultimately led to the motivation of this project which was to address the mechanical issues with DMO1 in order to offer a plausible conservative treatment solution by providing spinal distraction and mobility. The DMO1 prototype was designed in such a way that the flexible rod was used for both its distractive force properties as well as mobility-enabling properties. Because the mechanical issues with DMO1 arose when the user began to flex forward, it was believed that by redesigning DMO1 and decoupling the DFC and MEC and designing each component separately, the design limitations of DMO1 could be overcome. 

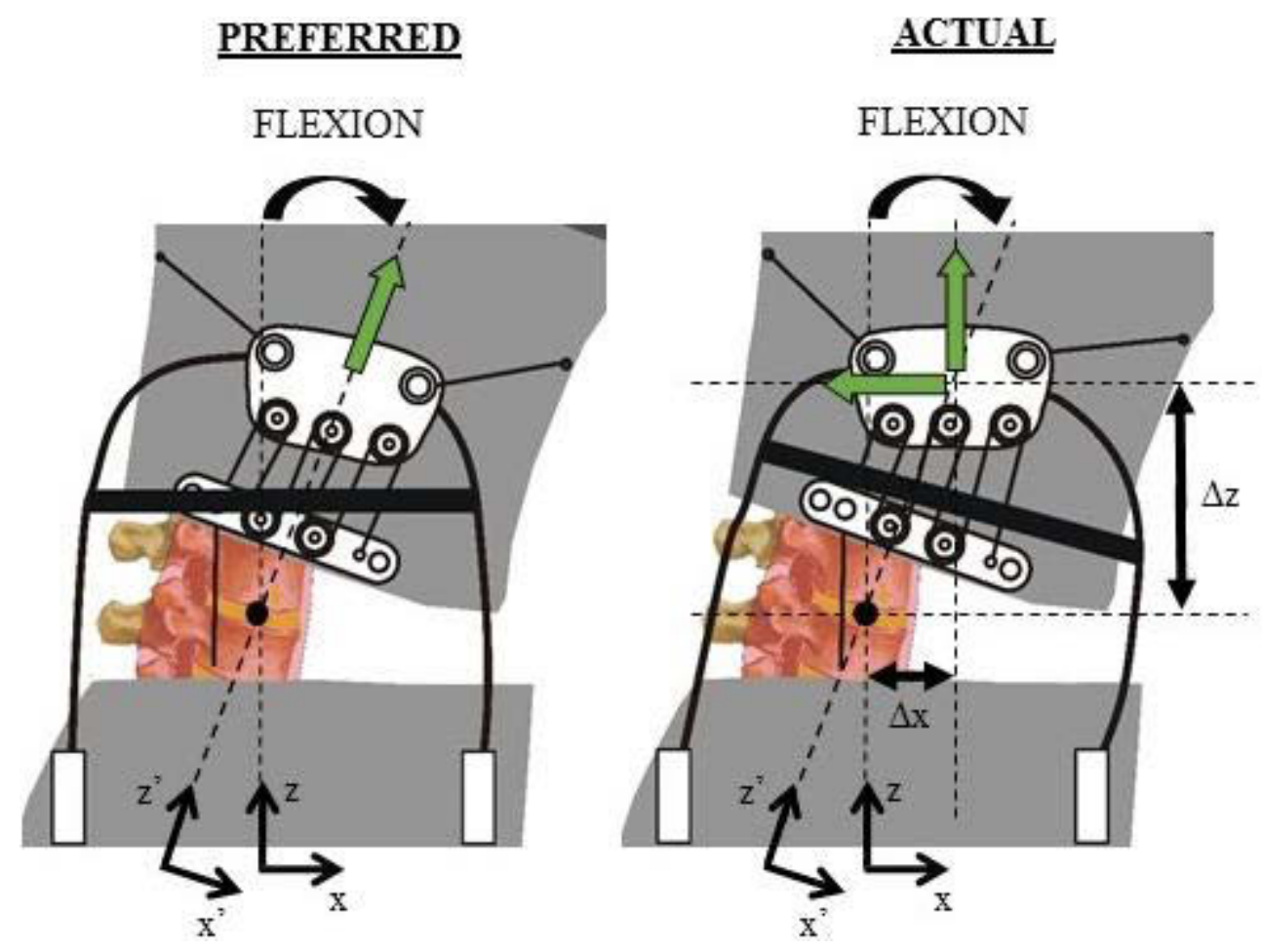

Figure 2-6. The preferred and actual behavior of the DMO1 MEC with a distractive force being applied 


\section{CHAPTER 3. METHODOLOGY*}

\section{Design and Laboratory Testing of DMO2}

The DMO1 prototype had previously proven to be effective in providing a distractive force across the lumbar spine throughout a limited range of extended flexion and extension. The limitations, as previously discussed, occurred when the device moved and additional resistance to bending was observed. Like DMO1, the design goals for the DMO2 prototype were the following [33]:

a) An effective body engagement mechanism. The DMO should be designed such that the pelvic belt remains secured to the iliac crest and the torso glove to the upper torso during the wearer's DLAs. Patients of different sizes should be able to wear the DMO.

b) A distractive force component. The DFC should provide a downward force on the iliac crest through the pelvic belt's engagement and an upward force on one's upper torso through the torso glove's engagement. As an upper limit, the DFC should fully support the torso weight of an average size, $170 \mathrm{lbs}$ human assuming his or her torso weight is $40 \%$ of the total body weight [34]. This upper limit that the DFC would be able to provide equates to about $300 \mathrm{~N}$.

c) A mobility-enabling component. The MEC should allow for movement (initially flexion and extension but potentially also axial rotation and lateral bending) with minimal resistance from the DMO. The targeted functional range of motion (ROM) of the DMO should be about 25 degrees of flexion and about 10 degrees of extension as most DLAs are encompassed within this range of motion [17].

d) Adjustment mechanisms. The adjustment mechanisms should include both a way to manually set both the distractive force that the DMO applies and the location of the rotational axis (RA) of the DMO. The RA should be approximately aligned with the CoR of the lumbar spine which previous work has shown to be located around the L3-L4 intervertebral disc [35].

Figure 3-1 outlines the design and evaluation process that was used for the DMO. As the design process illustrates, first an idea was conceptualized for a problem that has not been solved yet. After this, a design was proposed and then a prototype was built. Initial evaluation of the built prototype determined if the prototype warrants laboratory evaluation in a previously-established protocol for testing spinal orthoses [18]. After laboratory evaluation, one was able to determine if the design goals had been met. If so,

\footnotetext{
* Portions reprinted with permission from D. DiAngelo and D. Hillyard, "A novel distractive and mobility-enabling lumbar spinal orthosis," Journal of Rehabilitation and Assistive Technologies Engineering, vol. 3, pp. 1-10, 2016.
} 


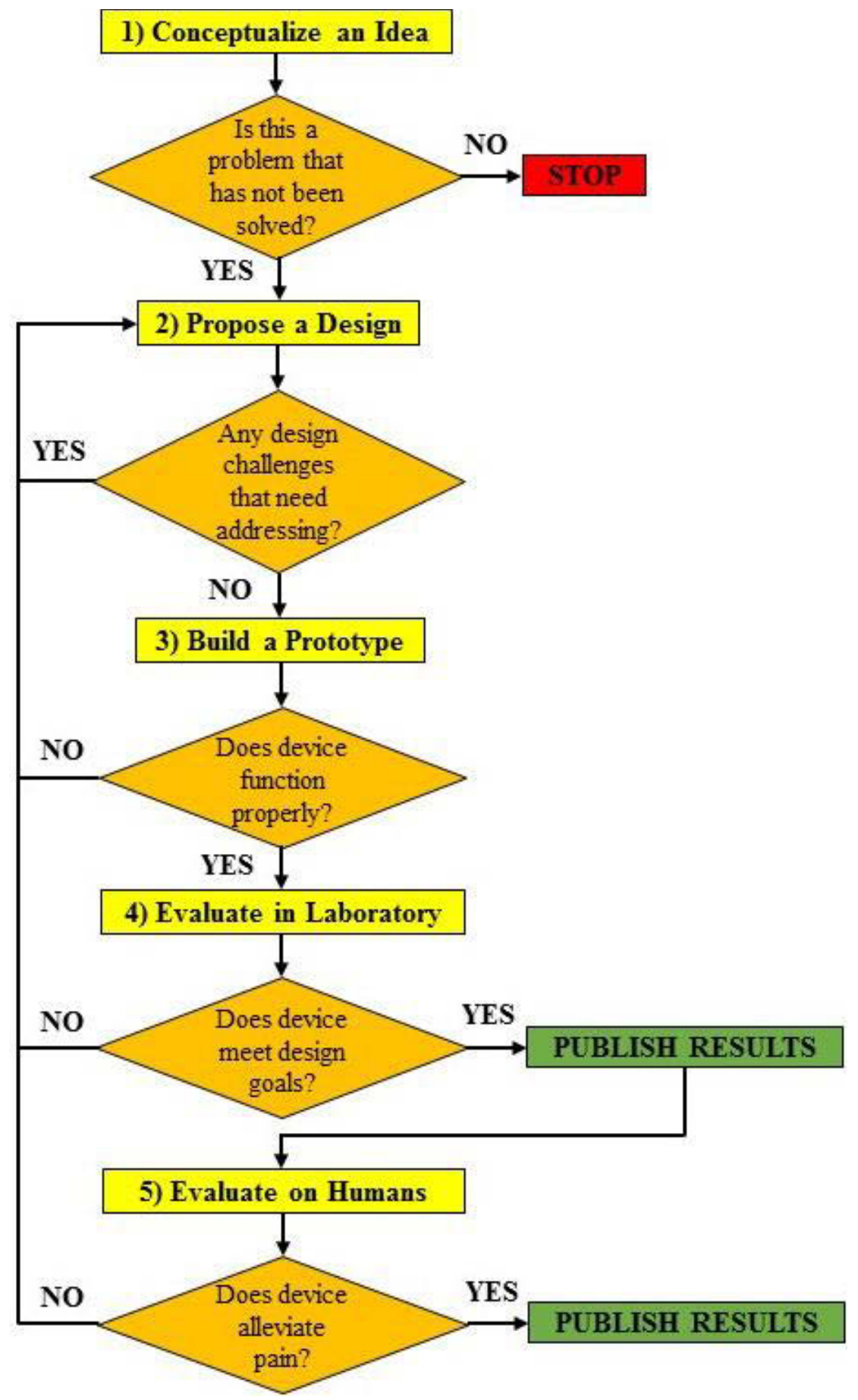

Figure 3-1. Flowchart of the design and evaluation process for the DMO 
one would publish the results and further test the device on human subjects. If the device alleviated pain for the human subjects, the design was proven to be a success and the results were published.

The design goal for the modified orthosis, DMO2, was to overcome the limitations of the original DMO1 prototype by sustaining spinal off-loading throughout extended ranges of flexion and extension with minimal buildup of the sagittal bending moment. Unlike the original DMO1 design where the function of the DFC and MEC components were coupled together, the modified design of DMO2, shown in Figure 3-2, had the two components function independent of each other.

\section{DMO2 Distractive Force Component}

The DFC consisted of a modified cable pulley system, a flexible graphite rod, a rod clamp, and a non-deformable ring as shown in Figure 3-3a. The ends of the graphite rod were anchored to the pelvic belt similar to the original orthotic design, and the upper section of the non-deformable ring attached to the torso glove by the use of tie rod ends. The top portion of the modified cable pulley system was rigidly fastened to the rod via a rod clamp and the lower part was connected to base of the non-deformable ring. When the modified cable pulley system was engaged, the rod clamp was pulled downward against the rod and deflected under load. With the rods anchored at the pelvic belt, an upward force was applied to the torso glove through the non-deformable ring. A band similar to that used in the DMO1 design was placed between the rods to control its flexural bending property. The non-deformable ring was translationally constrained by vertical guides and allowed to travel freely when the modified cable pulley system was engaged as shown in Figure 3-3b.

\section{DMO2 Mobility-Enabling Component}

The MEC consisted of three tie rod ends fastened to an interface plate and the non-deformable ring as shown in Figure 3-4. The interface plate attached to a mounting plate on the torso glove. The tie rod ends were guided by the non-deformable ring to provide flexion and extension. The RA of the MEC was adjustable by placing the interface plate on different attachment points on the mounting plate.

\section{Protocol for Testing and Evaluating DMO2 in the Laboratory Under Simulated Flexion and Extension}

The multi-axis robotic testing platform. A multi-axis robotic testing platform (RTP) [36] was used that provided four programmable degrees of freedom and having a positional resolution of $2 \mu \mathrm{m}$ in $\mathrm{x}, 0.31 \mu \mathrm{m}$ in $\mathrm{z}$, and $0.0002^{\circ}$ about $\mathrm{y}$ can be seen in

Figure 3-5a. The RTP had a six-axis load cell mounted to the upper gimbal assembly and another six-axis load cell mounted to the lower base plate. The upper load cell (ULC), 


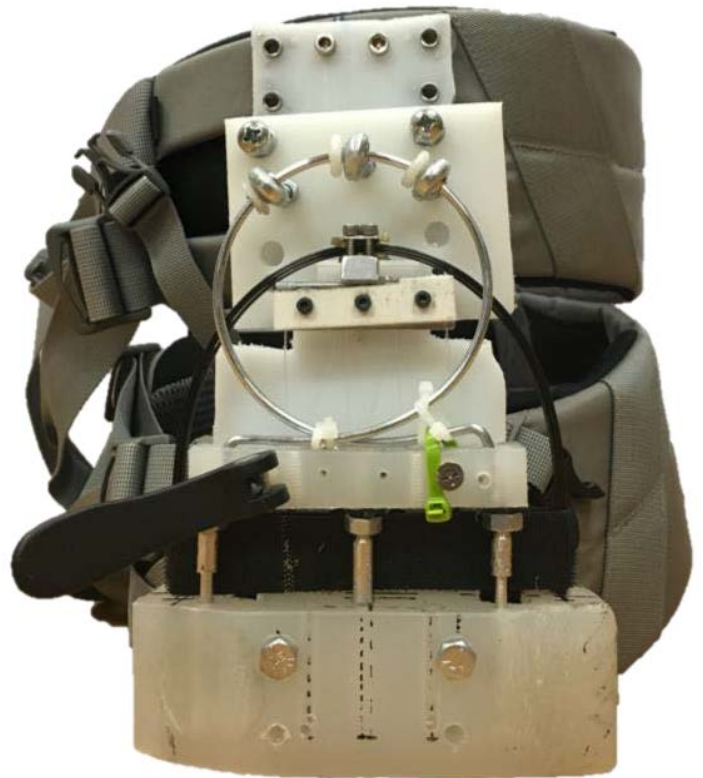

Figure 3-2. The DMO2 prototype 

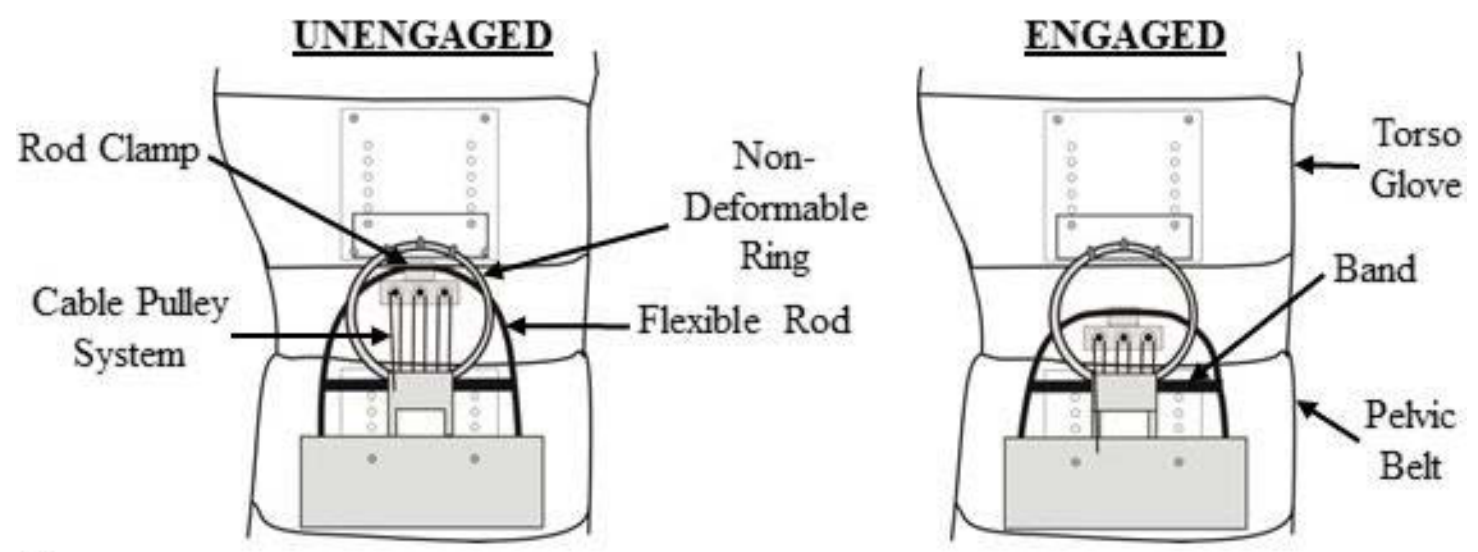

(a)

\section{UNENGAGED}
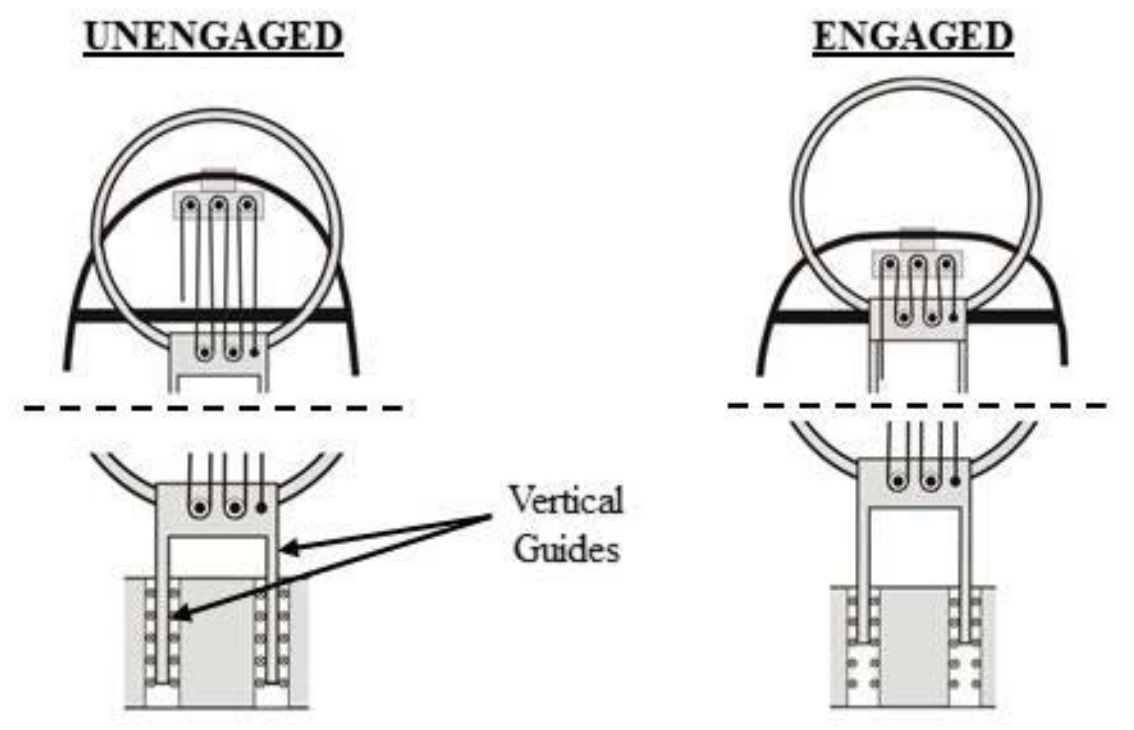

(b)

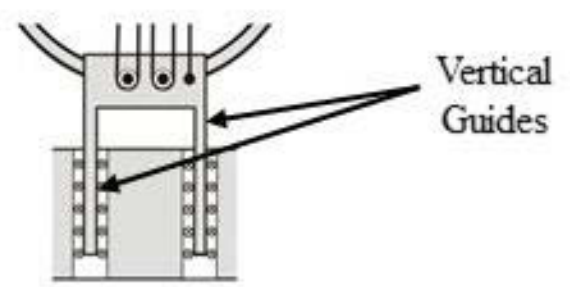

Figure 3-3. Operation of the distractive force component on DMO2

(a) Interaction between the cable pulley system, flexible rod, and non-deformable ring in the engaged and unengaged states

(b) The ring was permitted to move vertically in the engaged state 
VERTICAL STANCE

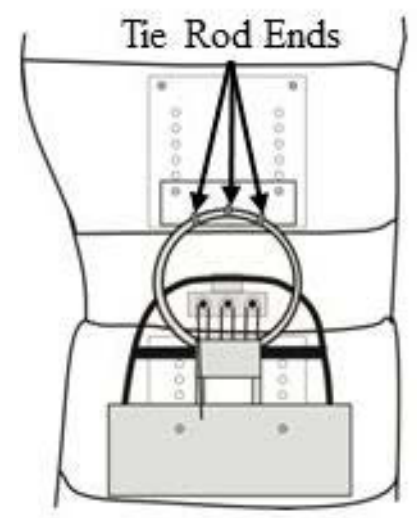

FLEXION

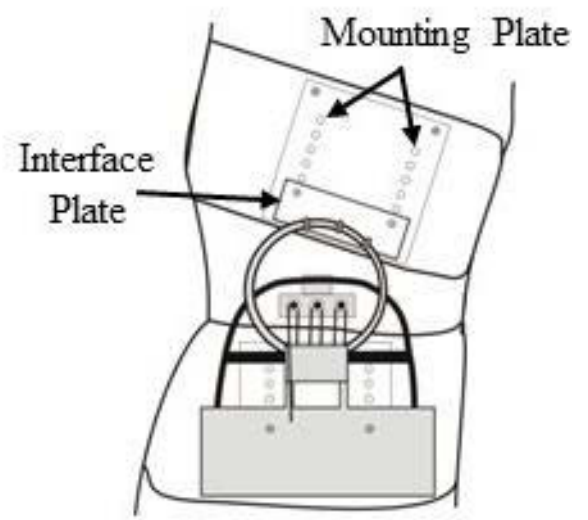

Figure 3-4. Operation of the mobility-enabling component on DMO2 


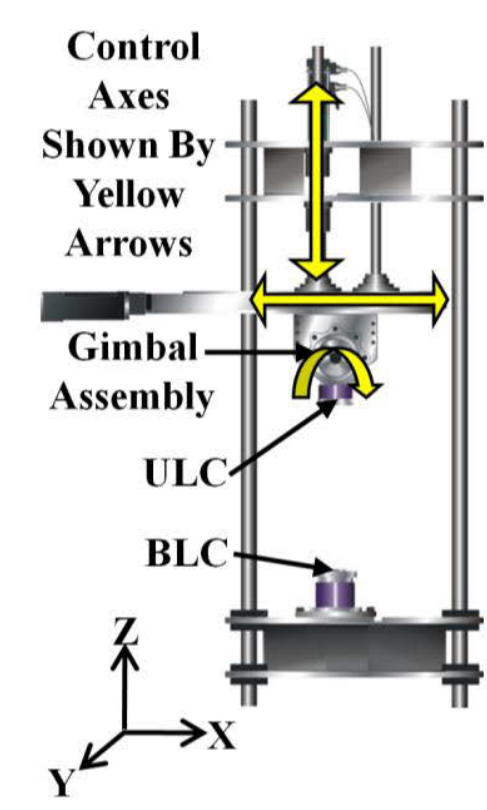

(a)

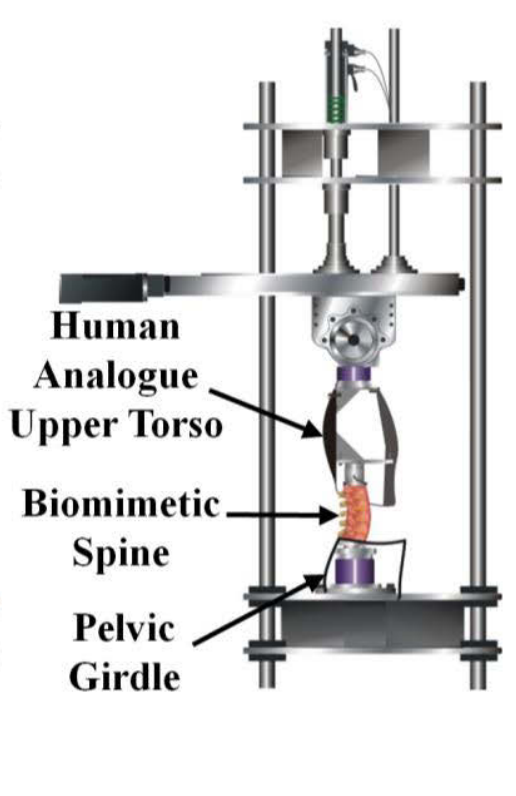

(b)

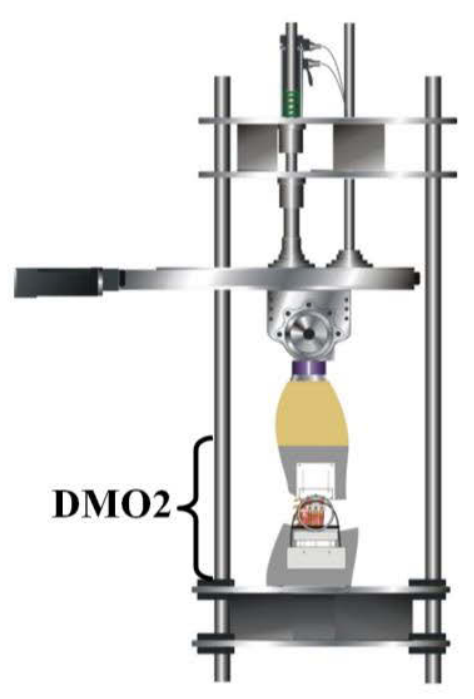

(c)

Figure 3-5. Advanced testing assembly
(a) RTP with programmable axes
(b) Human upper torso analog model, biomimetic spine, and pelvic girdle components mounted in the RTP
(c) Placement of DMO2 on the torso analog model mounted in the RTP 
which measured applied forces and moments, had a maximum axial force of $445 \mathrm{~N}$ and a resolution of $0.2 \mathrm{~N}$. The base load cell (BLC) had a maximum axial force of 4,445 $\mathrm{N}$ and a resolution of $0.73 \mathrm{~N}$.

The mechanical analog upper torso model. An upper torso, biomimetic lumbar spine, and pelvic girdle assembly (the combination of which is referred to as the human mechanical analog) was previously-designed to emulate the physical and structural properties of a male human adult torso. The biomimetic lumbar spine consisted of individual spinal components having shape and size comparable to the human lumbar motion segments. The L1-L5 vertebral bodies were cast in rubber molds made from harvested human spines. The individual discs were previously-fabricated based on characteristics from the literature [37] and provided the anterior and posterior heights for each disc. The material for each disc is 30 durometer urethane (74-30D Urethane from US Composites, 6670 White Drive West Palm Beach, FL 33407). The full L1-L5 lumbar assembly was previously-coated with 30 durometer urethane. The final flexural rotational stiffness over $10^{\circ}$ of flexion was $0.66 \mathrm{Nm} /{ }^{\circ}$ which approximated cadaveric test data [35].

A life size male mannequin was previously-cut and substantially reinforced internally with carbon fiber and epoxy resin to provide an upper torso frame and separate pelvic girdle assembly for engaging a worn orthosis as it was tested. Multiple layers of a textured material (Kobalt Zerust drawer liner, Zerust Corrosion Products. Twinsburg, OH 44087) were previously-placed around the external surface of the upper torso component that simulated the texture and orthosis-engagement properties of human tissue. The material had a hardness of approximately 30 Durometer Shore A at its thickest section of weave pattern. Each layer was previously-impregnated and externally coated with a thin coating of 30 Durometer Shore A urethane. The upper torso provided engagement for the torso glove of the orthosis to be tested. The biomimetic spine was mounted superiorly to the upper torso frame and inferiorly to the BLC as seen in Figure 3-5b. The BLC was surrounded by, but not in contact with, the pelvic girdle assembly. The pelvic girdle assembly was provided to mount to and engage the pelvic belt of the orthosis to be tested and was anchored to the base plate of the RTP.

DMO2 was placed on the mechanical analog upper torso model that was mounted in the robotic testing platform and collectively used to design unique features of the proposed dynamic orthosis Figure 3-5c. The location of the non-deformable ring on DMO2 was adjustable with the goal of aligning the center of the ring close to the center of rotation (CoR) of the lumbar spine as shown in Figure 3-6. If the center of the ring was not aligned properly with the CoR of the lumbar spine, the distractive force from the DFC created loads off-axis to the CoR of the spine ( $\mathrm{dx}^{\prime}$ and $\mathrm{dz}$ ') that contributed additional bending moments about the spine. By correctly aligning the center of the ring close to the CoR of the lumbar spine, minimal additional bending moment was required to move. Correct alignment of the center of the ring with the CoR of the lumbar spine ensured that DMO2 and the lumbar spine were working together during extended ranges of flexion and extension. 

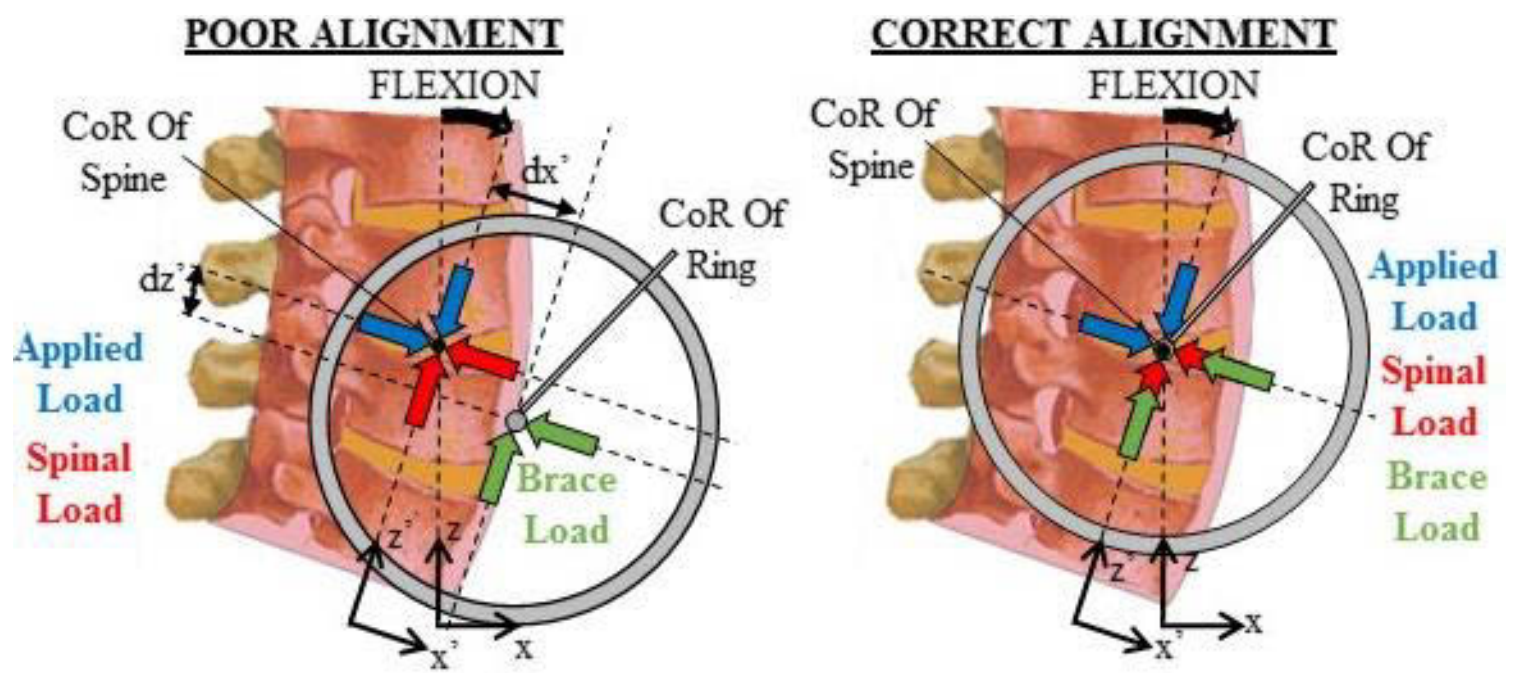

Figure 3-6. Proper placement of the rotational axis of the DMO relative to the lumbar spine 
Testing protocol and force analysis. The test conditions for the DMO2 prototype were similar to that used in the original evaluation of DMO1: an upper torso load of $300 \mathrm{~N}$ in upright stance, initiation of flexion and extension, and extended ranges of $25^{\circ}$ flexion and $10^{\circ}$ extension $[16,18]$. The $300 \mathrm{~N}$ value simulated the upper body (above the abdomen) weight of a person whose approximate total body weight was $750 \mathrm{~N}$ based on the anthropometric data that the upper body comprised approximately $40 \%$ of total body weight [34]. In the end, the loads applied to the spine and orthosis consisted of a bending moment and the upper body weight force components. To apply these loading conditions, the RTP was first programmed to establish the kinematic path of the lumbar spine alone under pure moment loading by introducing an incremental rotation to the spine and then reducing the off-axis forces by minimizing the distance ( $\Delta x^{\prime}$ and $\left.\Delta z^{\prime}\right)$ between the initial prescribed $\mathrm{CoR}$ and true CoR of the lumbar spine which can be seen in Figure 3-7. Once the location for a pure moment loading condition was established at every $0.5^{\circ}$ incremental rotation, the force components of the upper body weight could be applied along the new rotational axes until the end rotation limit was reached to simulate the loading conditions of a bending moment plus upper body weight forces. Data from the modified kinematic path of the robot was also used to determine the CoR of the biomimetic lumbar spine by adapting the CoR equations from Crisco [38]. Note that these CoR values of the lumbar spine were used above to align the center of the nondeformable ring on DMO2 with the CoR of the lumbar spine. The modified orthosis was then mounted on the testing platform, aligned with the spine, and the advanced testing protocol was rerun to simulate the testing conditions above.

The primary outcomes for DMO2 laboratory testing were spinal off-loading (brace load) and bending moment (brace effect) at upright stance and during flexion and extension ranges. Applied loads to the torso-orthosis assembly by the RTP were measured at the ULC and the loads transferred through the lumbar spine were measured at the BLC. The ULC and BLC forces and bending moments (BM) were transformed to the sacral disc plane (SDP) and compared throughout flexion and extension as seen in

Figure 3-8a. The difference in the applied load and the transferred load represented the brace load carried by DMO2 as shown in Figure 3-8b. Figure 3-8c illustrates how the difference in the applied moment and the transferred moment represented the brace effect moment caused by DMO2.

\section{Design and Laboratory Testing of DMO3}

The design goal for the DMO3 prototype was to overcome the limitations of the DMO2 prototype by sustaining spinal off-loading throughout extended ranges of flexion and extension with minimal buildup of the sagittal bending moment. Also, an effort was made to incorporate the ability of the DMO3 prototype to allow for both axial rotation and lateral bending. The design goals for the DMO3 prototype were the following: 

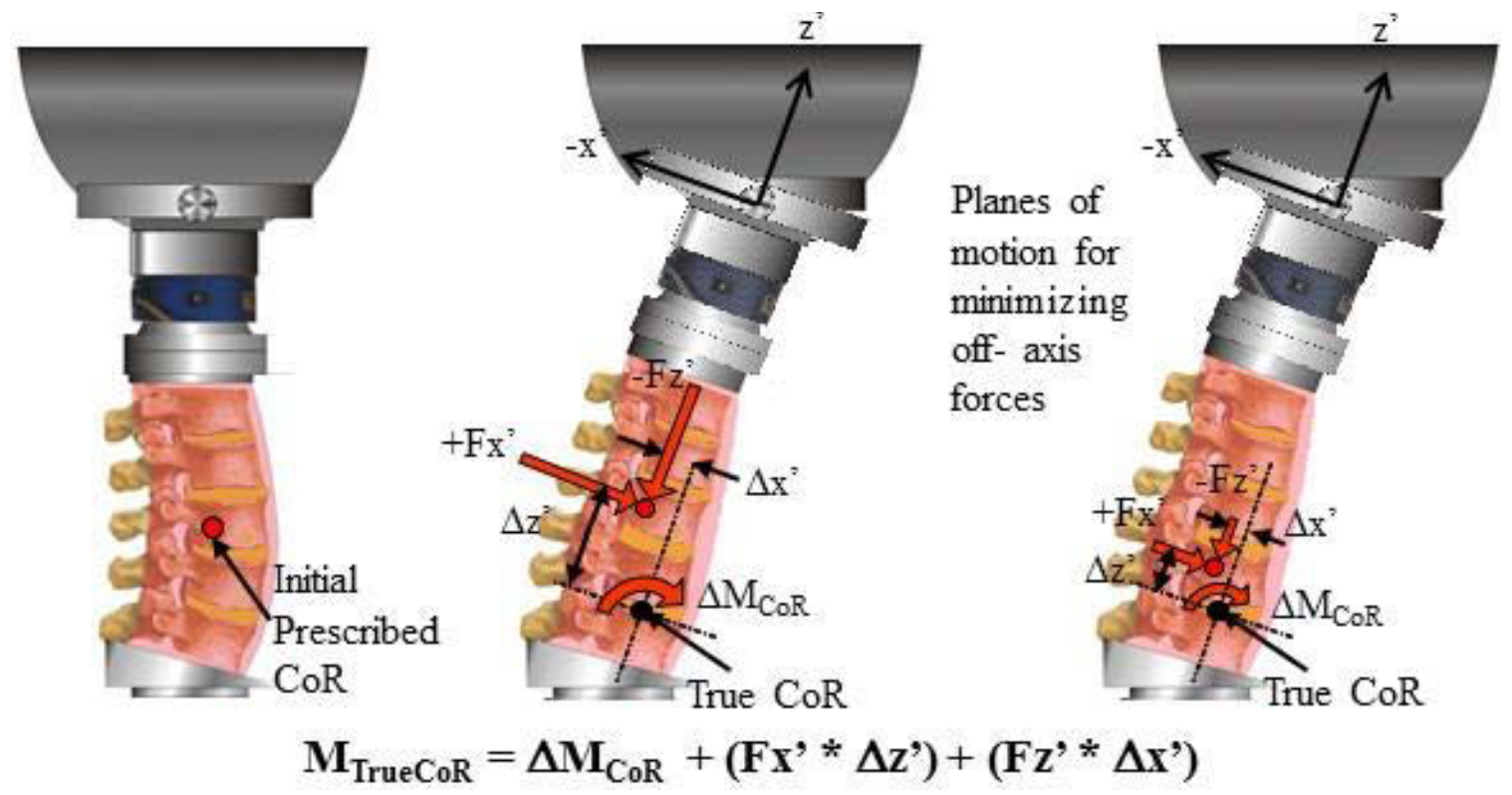

Figure 3-7. Advanced control strategy for minimizing off-axis force contribution to the lumbar spine at each incremental rotation 

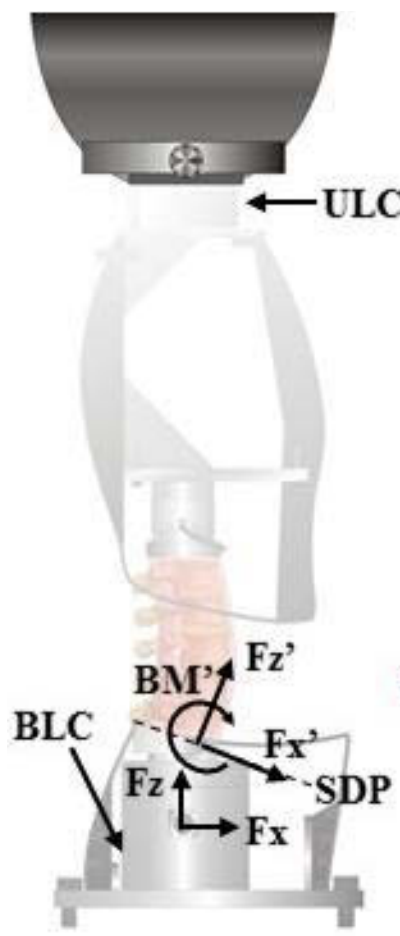

(a)

(b)

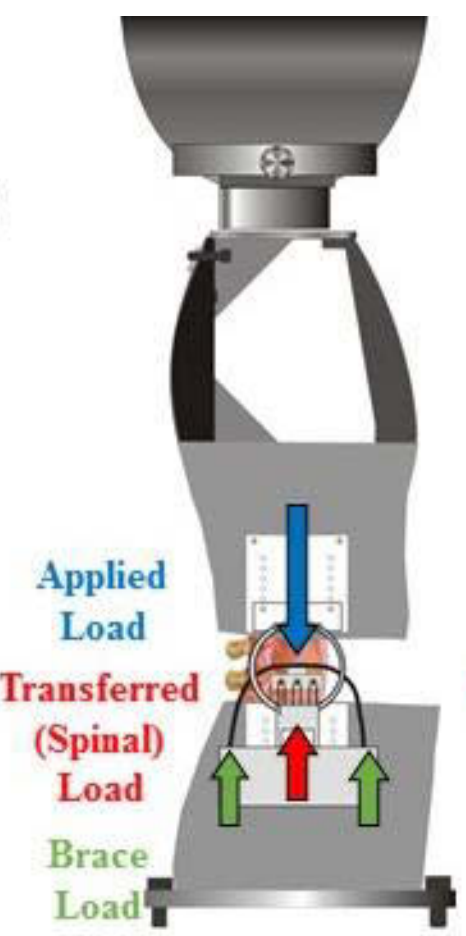

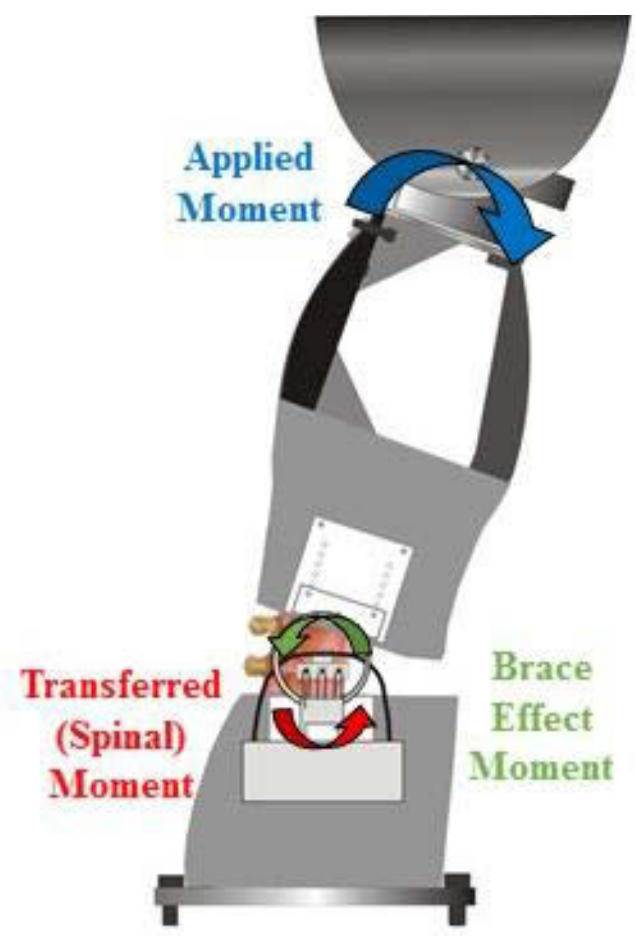

(c)

Figure 3-8. Force analysis of the DMO2 prototype in the RTP

(a) Forces were transformed to the SDP

(b) The brace load was calculated as the difference in the measured applied load and the transferred load

(c) The brace effect was calculated as the difference in the measured applied moment and the transferred moment 
a) An effective body engagement mechanism. The DMO3 should be designed such that the pelvic belt remains secured to the iliac crest and the torso belt and torso glove to the wearer's torso during their DLAs. Patients of different sizes should be able to wear DMO3.

b) A distractive force component. The distractive force component (DFC) should provide a downward force on the iliac crest through the pelvic belt's engagement and an upward force to one's torso through the torso belt and torso glove's engagement. The initial prototype will be designed to support half of the torso weight of an average male individual weighing $750 \mathrm{~N}$ (or $170 \mathrm{lbs}$ ) with a torso weight of $40 \%$ of the total body weight [34]. This upper limit that the DFC would be able to provide equates to about $150 \mathrm{~N}$. This force is to be applied and maintained throughout extended ranges of motion.

c) A mobility-enabling component. The MEC should allow for movement flexion, extension, and axial rotation movement with minimal resistance from DMO3. As shown in Figure 3-9, the targeted functional range of motion (ROM) of DMO3 should be about 25 degrees of flexion, 10 degrees of extension, and 10 degrees of axial rotation as most DLAs are encompassed within this range of motion [17].

d) Adjustment mechanisms. The adjustment mechanisms should include both a way to manually set both the distractive force that DMO3 applies and the location of the rotational axis (RA) of DMO3. The RA should be approximately aligned with the CoR of the lumbar spine which previous work has shown to be located around the L3-L4 intervertebral disc [35].

Figure 3-10a shows the engagement components of DMO3. An extra engagement component was added to the DMO3 prototype compared with both DMO1 and DMO2. For DMO3, a neoprene vest was the torso glove. The advantage of adding this neoprene vest was that it can engage more of the wearer's surface area and prevent slippage of the device on the wearer's torso. The torso belt, previously referred to as the torso glove in the DMO2 prototype design, would wrap around the wearer's torso after the wearer had donned the torso glove. The interior of the DMO3 torso belt was layered with loop Velcro material that attached to the hook Velcro material on the exterior of the DMO3 torso glove. No changes were made to the pelvic belt of the DMO3 prototype. The pelvic belt had an iliac pad on the left and ride interior sides of the belt that allowed for comfortable, secure engagement to the wearer's iliac crest.

The mechanism by which a distractive force was applied to the wearer of DMO3 is referred to as the distractive force component (DFC), as shown in Figure 3-10b. This mechanism was placed on the left and right sides of the wearer and connected the superior engagement components of the DFC (torso glove) with the inferior engagement components (pelvic belt). The method by which the DFC attaches to the torso belt and pelvic belt will be outlined in future sections. 


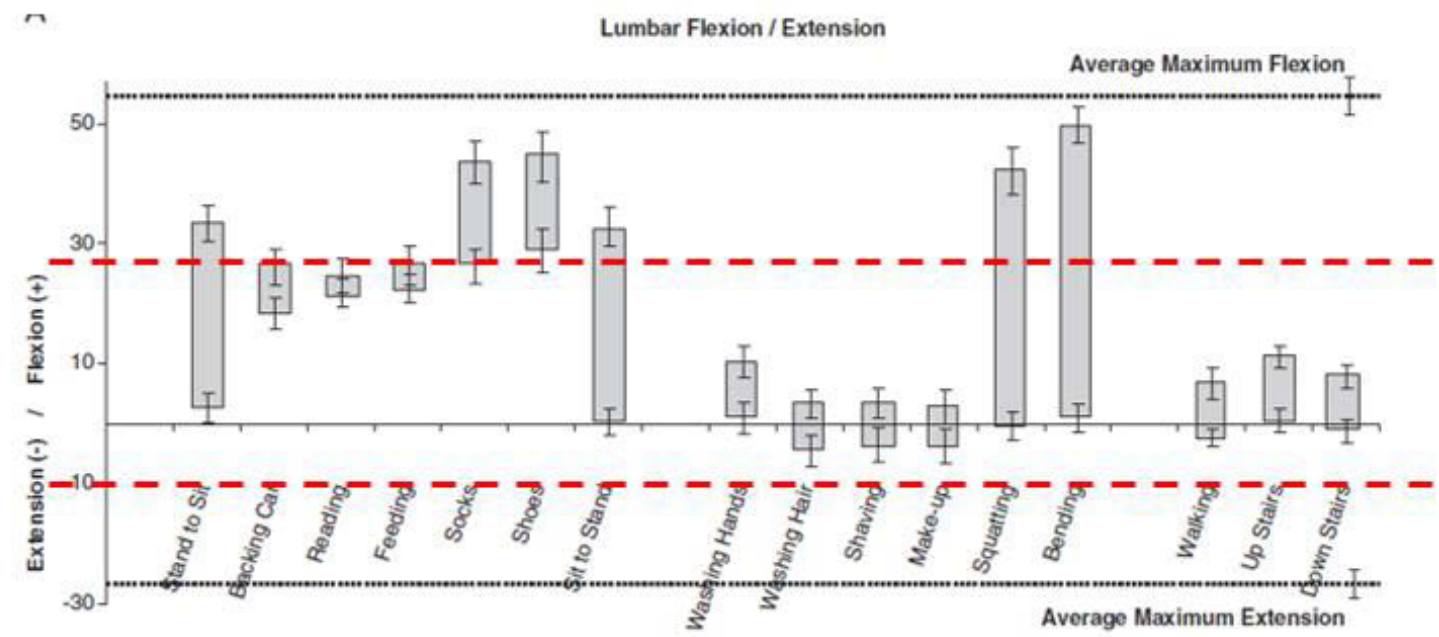

(a)

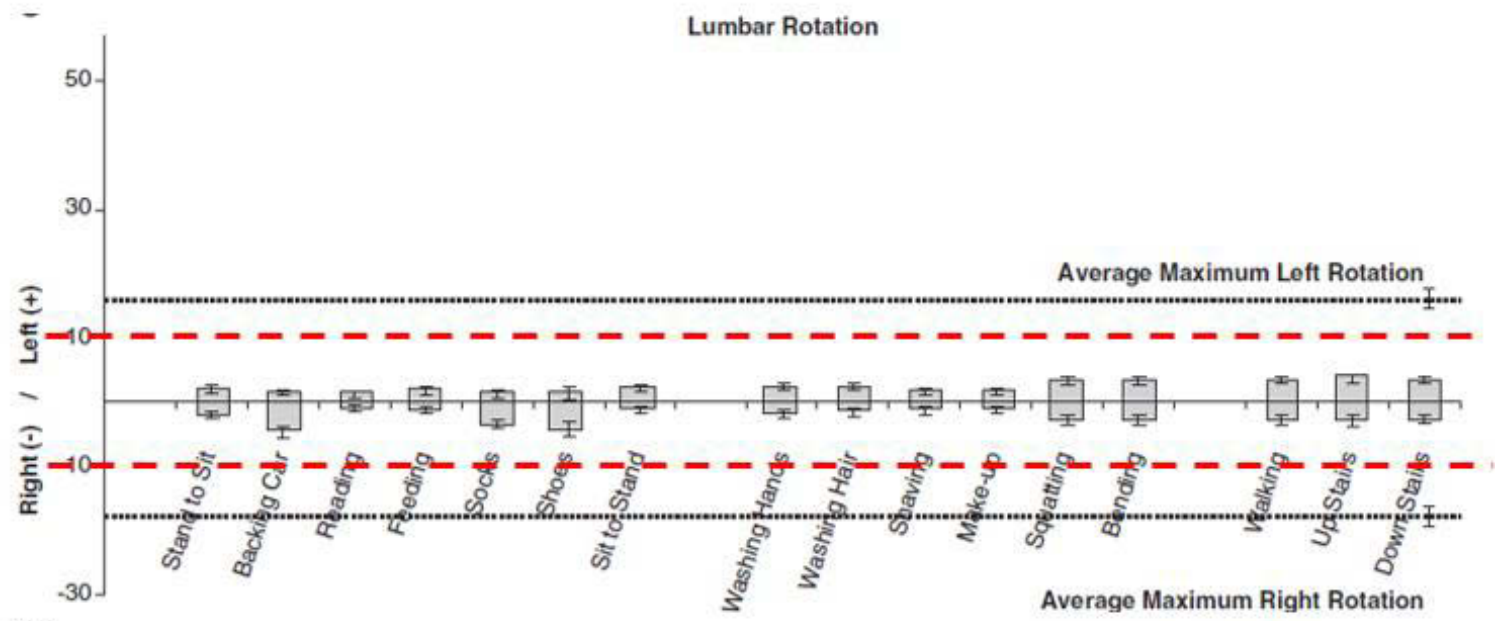

(b)

Figure 3-9. A measure of the functional ROM of the lumbar spine in flexion, extension, and axial rotation of the DMO3 prototype

Reprinted with permission. J. Bible, "Normal functional range of motion of the lumbar spine during 15 activities of daily living," J Spinal Disord Tech, vol. 23, pp. 106-112, 2010. 


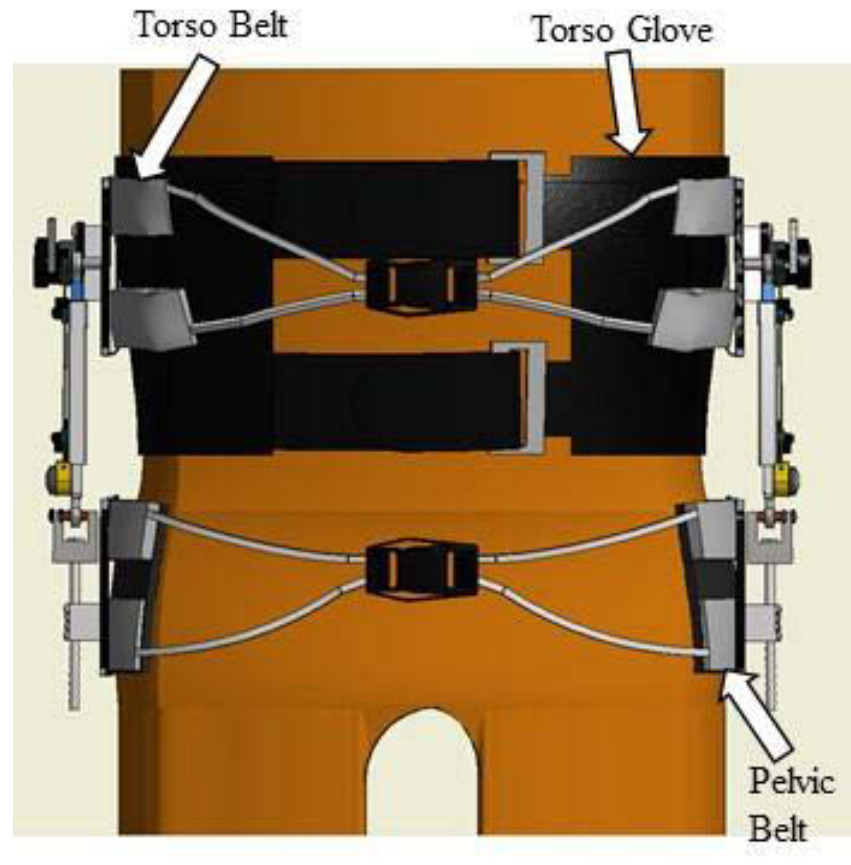

(a)

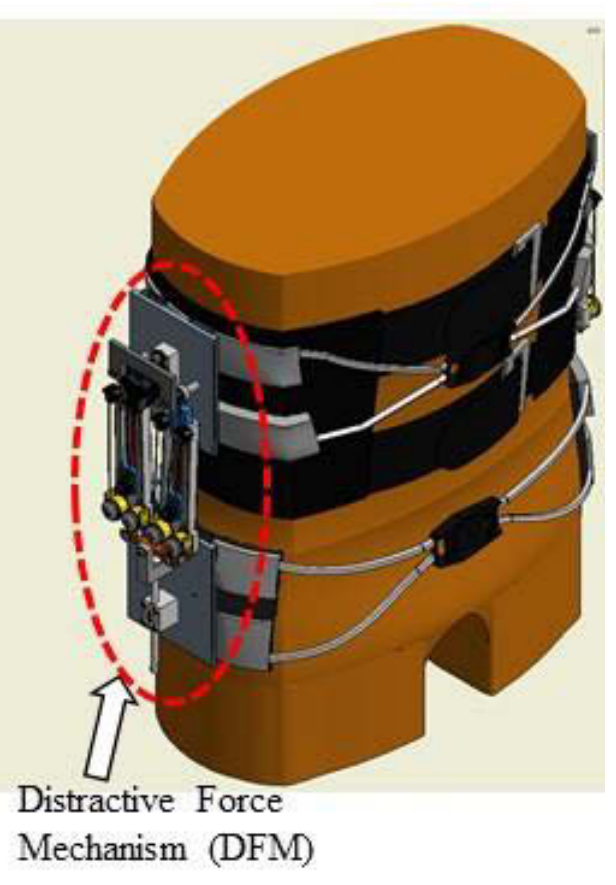

(b)

Figure 3-10. The new DMO3 prototype 


\section{DMO3 Body Engagement Mechanisms}

The torso belt, which can be seen in Figure 3-11, was a modified version of the Osprey Isoform Men's Hipbelt (Osprey Packs, Inc., CO, USA) which is commonly used with Osprey hiking backpacks. It was utilized to engage the wearer's torso by wrapping around the body and conforming to the wearer's natural anatomical profile. Hook Velcro (1) attached to the inside surface of the torso belt which enabled secure engagement to the torso glove. Thermal moldable foam (2), such as ethyl vinyl acetate, comprised the main structural component. The torso belt could be placed inside an oven heated at approximately $200^{\circ} \mathrm{F}$ for ten minutes. After heating, one may wrap the belt around the torso until cooled to ensure that the belt was contoured to the specific torso anatomy of the wearer. The torso belt was secured to the wearer by a buckle (3) and four Dacron straps (4) which were located ventrally on the wearer. Two Dacron straps (4) were attached to each opening end of the torso belt and allowed for the belt to be tightened on the wearer when it was secured to the wearer. When the Dacron straps (4) were tightened, both the upper and lower sections of the torso belt became more secured to the wearer simultaneously. The buckle (3) allowed for the wearer to easily don and doff the torso belt.

The torso glove, as shown in Figure 3-12, was designed to engage the wearer's torso securely without slipping. The main component was neoprene rubber (5) that made up the inside surface of the torso glove. The outside surface was lined with loop Velcro (6) which provided the attachment site for the hook Velcro (1) of the torso belt to be attached. Donning and doffing of the torso glove was made possible through two plastic loops (7) and two Velcro straps (8) that are attached to each opening end of the torso glove. The two Velcro straps (8) went through their respective plastic loops and then attached to itself through hook and loop Velcro. When the Velcro straps (8) were tightened, the neoprene rubber (5) stretched so that the Torso Glove (B) became very securely engaged to the wearer's torso.

The pelvic belt, as shown in Figure 3-13, was the Osprey Isoform Men's Hipbelt (Osprey Packs, Inc., CO, USA). It was utilized to engage around the wearer's hips and conforming to the wearer's natural anatomical profile. An iliac pad (9) was located on the left and right interior lateral sides of the pelvic belt. This iliac pad (9) was intended to be located on the wearer's iliac crest. Thermal moldable foam (2), such as ethyl vinyl acetate, comprised the main structural component. The pelvic belt could be thermally molded to the wearer's anatomy in the same way that the torso belt was molded. The pelvic belt was secured to the wearer by a buckle (3) and four Dacron straps (4) which were located ventrally on the wearer. Two Dacron straps (4) were attached to each opening end of the pelvic belt (C) and allowed for the belt to be tightened on the wearer when it was secured to the wearer. When the Dacron straps (4) were tightened, both the upper and lower sections of the pelvic belt became more secured to the wearer simultaneously. The buckle (3) allowed for the wearer to easily don and doff the pelvic belt. 

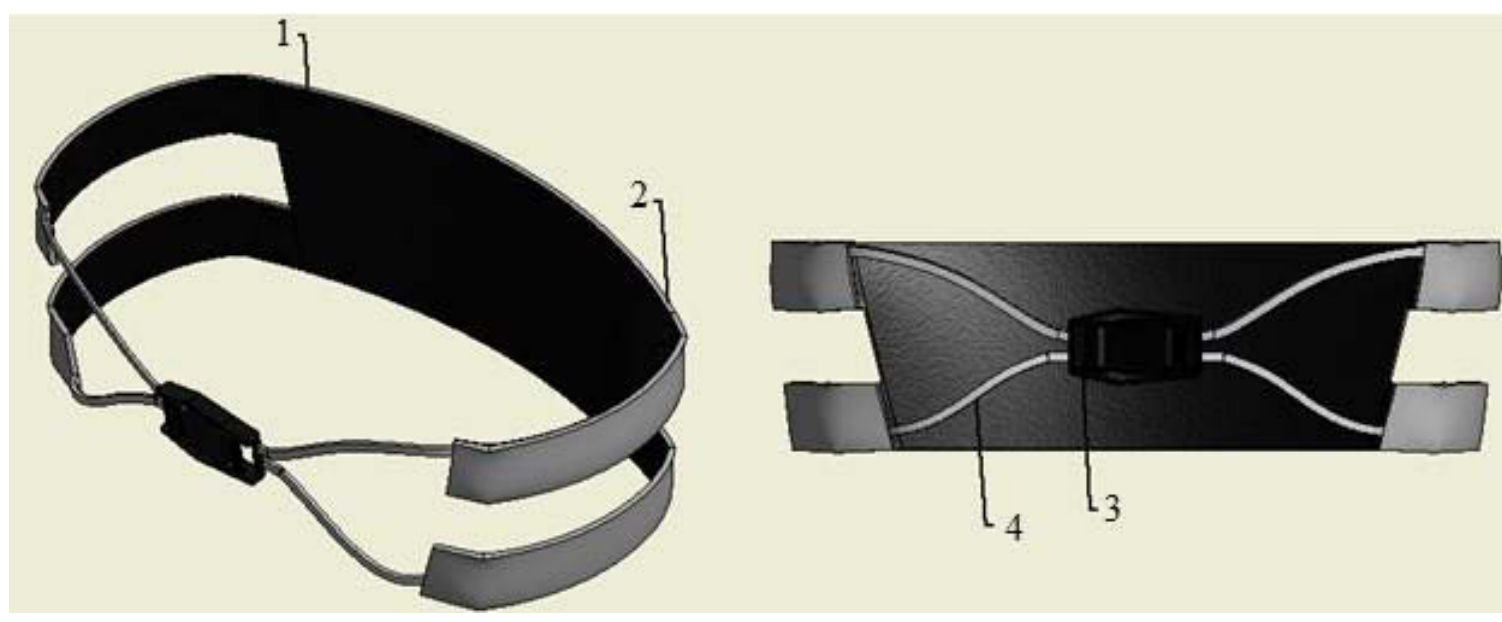

Figure 3-11. The DMO3 torso belt 

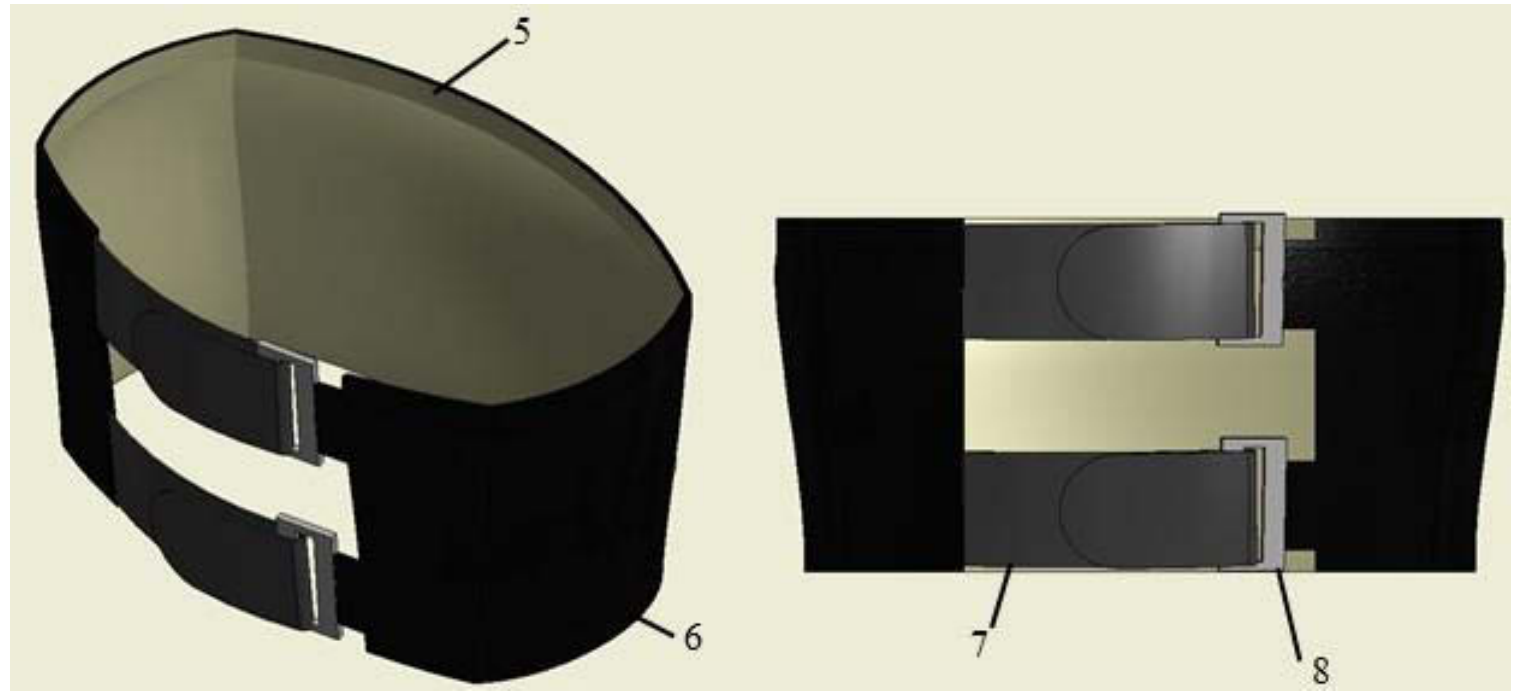

Figure 3-12. The DMO3 torso glove


Figure 3-13. The DMO3 pelvic belt 


\section{DMO3 Distractive Force Mechanism}

The distractive force component (DFC), as shown in Figure 3-14, was composed of the torso belt assembly, two mechanical actuators, the pelvic belt assembly, and a cable tensioning system (CTS).

Figure 3-15 shows how the torso belt assembly attaches to the torso belt and Figure 3-16 highlights the components of the torso belt assembly. The torso belt assembly attached to the torso belt by wrapping two Velcro straps (8) around the torso belt, passing them through metal loops (10), and fastening the Velcro strap (8) on itself. The Velcro straps (8) and metal loops (10) were attached to a fabric sleeve (11) that housed a plastic attachment plate (12) to which all of the torso belt assembly components attached. A tensioning knob (13) was separated from the plastic attachment plate (12) by a tensioning knob spacer (14) and attached in the middle of the plastic attachment plate (12) by connectors (15). Cable 2 (16) of the CTS was routed from the tensioning knob (13) and around a pulley (17) located on both sides of the tensioning knob (13) and appropriately spaced from the plastic attachment plate (12) with spacers (18). Cable 2 (16) was guided on the pulley (17) and prevented from coming off by locating a pulley guard (19) and a washer (20) on both sides of the pulley (17). A bolt (21) passed through all components on each side of the tensioning knob (13) and attached to the plastic attachment plate (12). A bracket (22) was placed on both bolts to prevent the bolts from bending inward.

Each mechanical actuator, whose components can be seen in Figure 3-17, attached superiorly to a bolt (21) on the torso belt assembly and inferiorly to a bolt (21) on the pelvic belt assembly via a tie rod end (23). The primary component of each mechanical actuator was a c-channel (24) to which all components were attached to or constrained by. A channel slot nut (25) was guided by the c-channel (24) for translation of two aluminum rods (26) as shown in Figure 3-18. The superior end of each aluminum rod (26) was attached to a plastic adapter (27), and the inferior end was attached to the channel slot nut (25) through bolts (21). Two constant force springs (CFS, 28) were connected to the inferior end of the c-channel (24) and a washer (20) was placed on each side of each CFS (28). Cable 1 (29) of the CTS was routed from each CFS (28) and around a pulley (17) located on both superior ends of the c-channel (24) before attaching to a washer (20). Cable 1 (29) was guided on the pulley (17) and prevented from coming off by locating a pulley guard (19) and the c-channel (24) on both sides of the pulley (17).

Figure 3-19 shows how the pelvic belt assembly attached to the pelvic belt and Figure 3-20 highlights the components of the pelvic belt assembly. The pelvic belt assembly attached to the pelvic belt by wrapping two Velcro straps (8) around the pelvic belt, passing them through metal loops (10), and fastening the Velcro strap (8) on itself. The Velcro straps (8) and metal loops (10) were attached to a fabric sleeve (11) that housed a plastic attachment plate (12) to which all of the components attach. A plastic receiver (30) was attached to the plastic attachment plate (12) through bolts (21). An axis adjustment bar (31) passed through a rod clevis (32) and attaches to the pelvic belt 


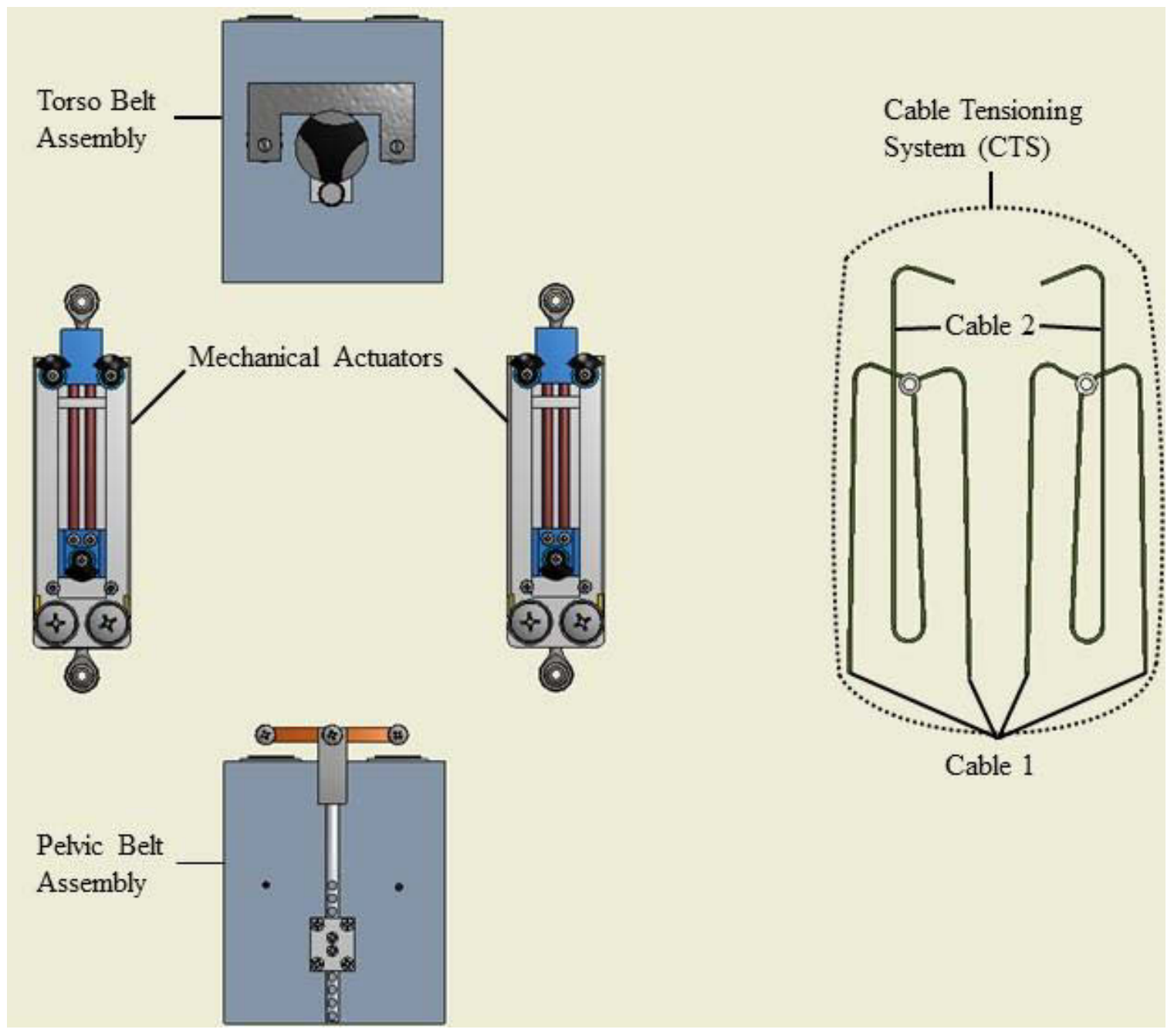

Figure 3-14. The four components of the distractive force component of DMO3 were the torso belt assembly, two mechanical actuators, the pelvic belt assembly, and a cable tensioning system 
Isometric (Exterior) View

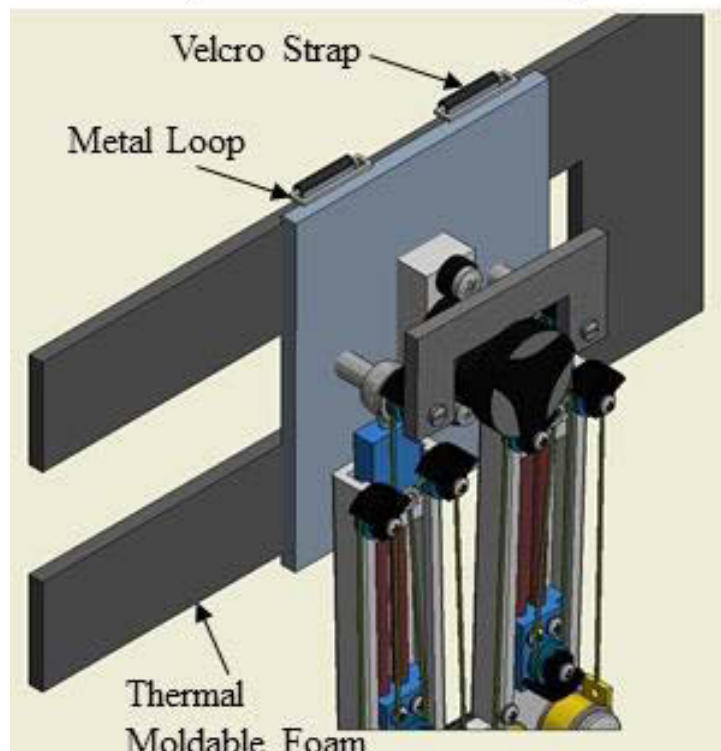

$\underline{\text { Isometric (Interior) View }}$

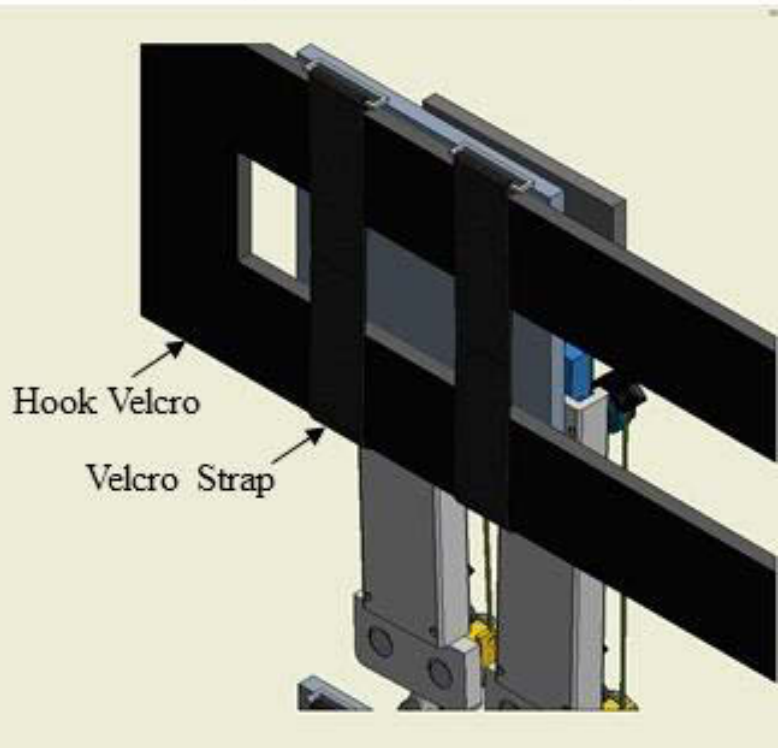

Figure 3-15. The torso belt assembly attaches to the torso belt by use of two Velcro straps and metal loops

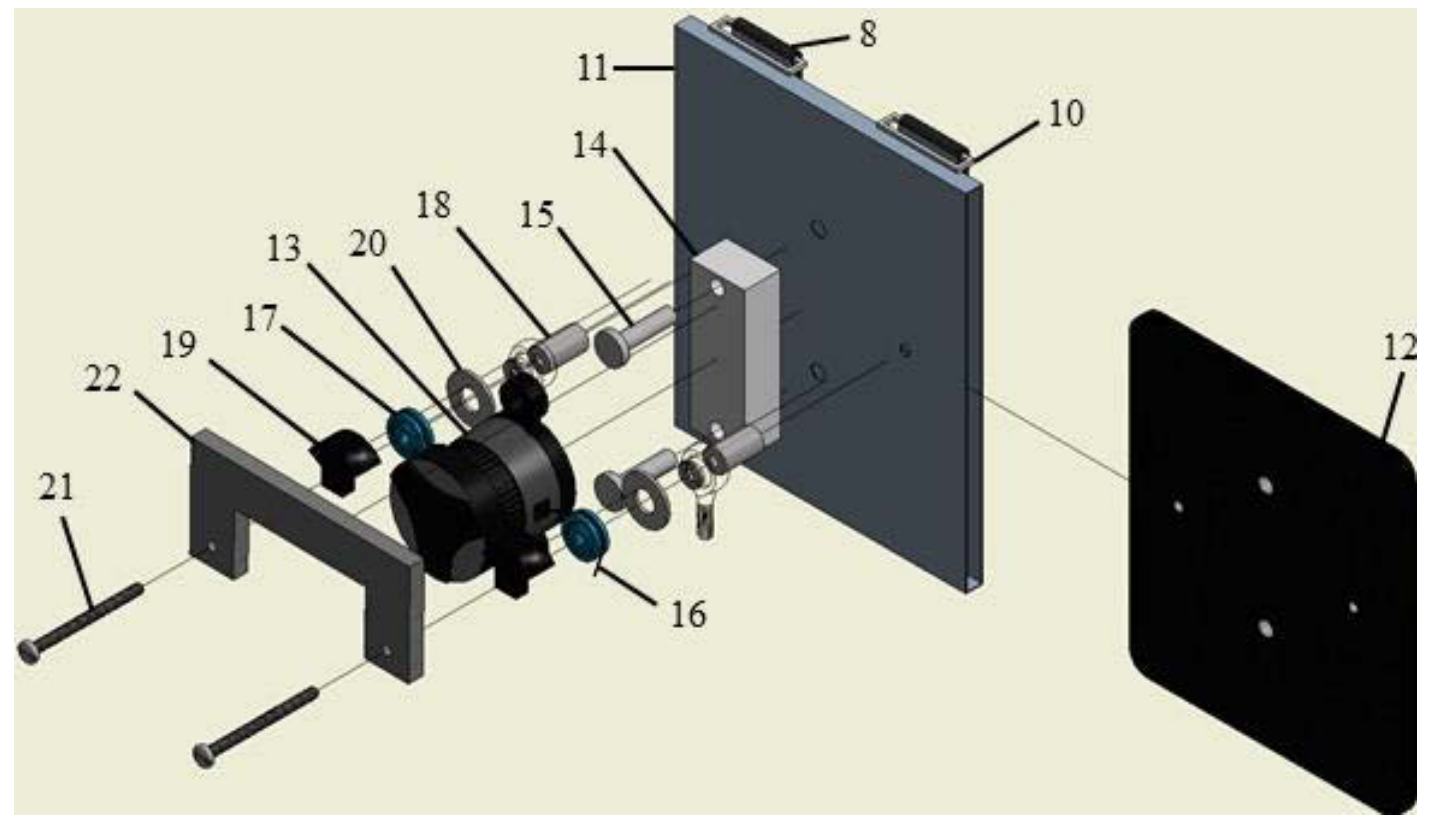

Figure 3-16. The components of the torso belt assembly 


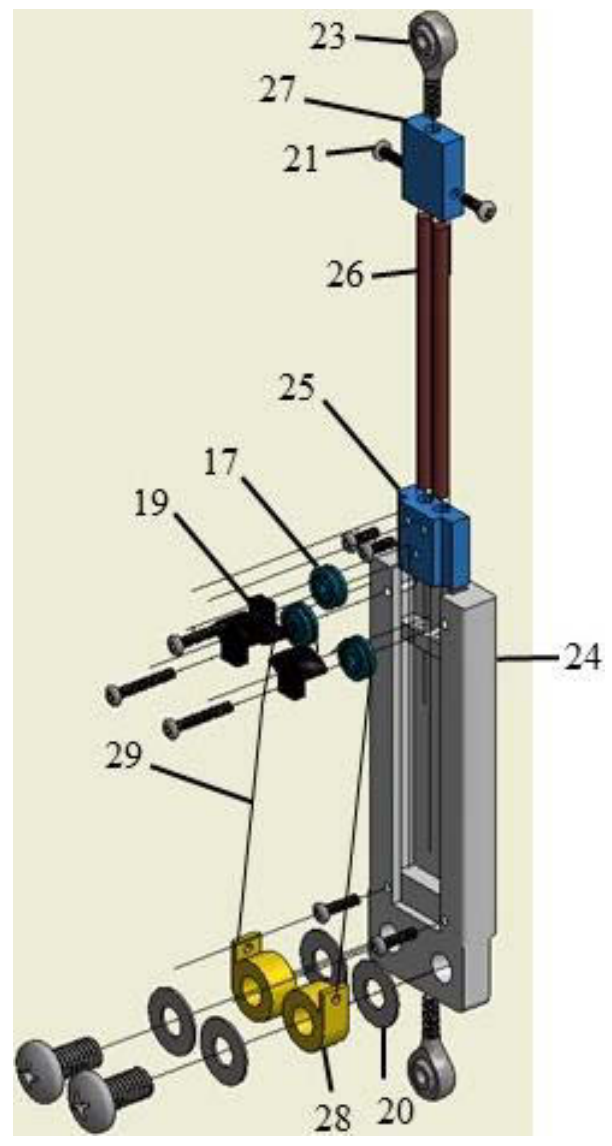

Figure 3-17. The components of each mechanical actuator 
UNEXTENDED

Front View

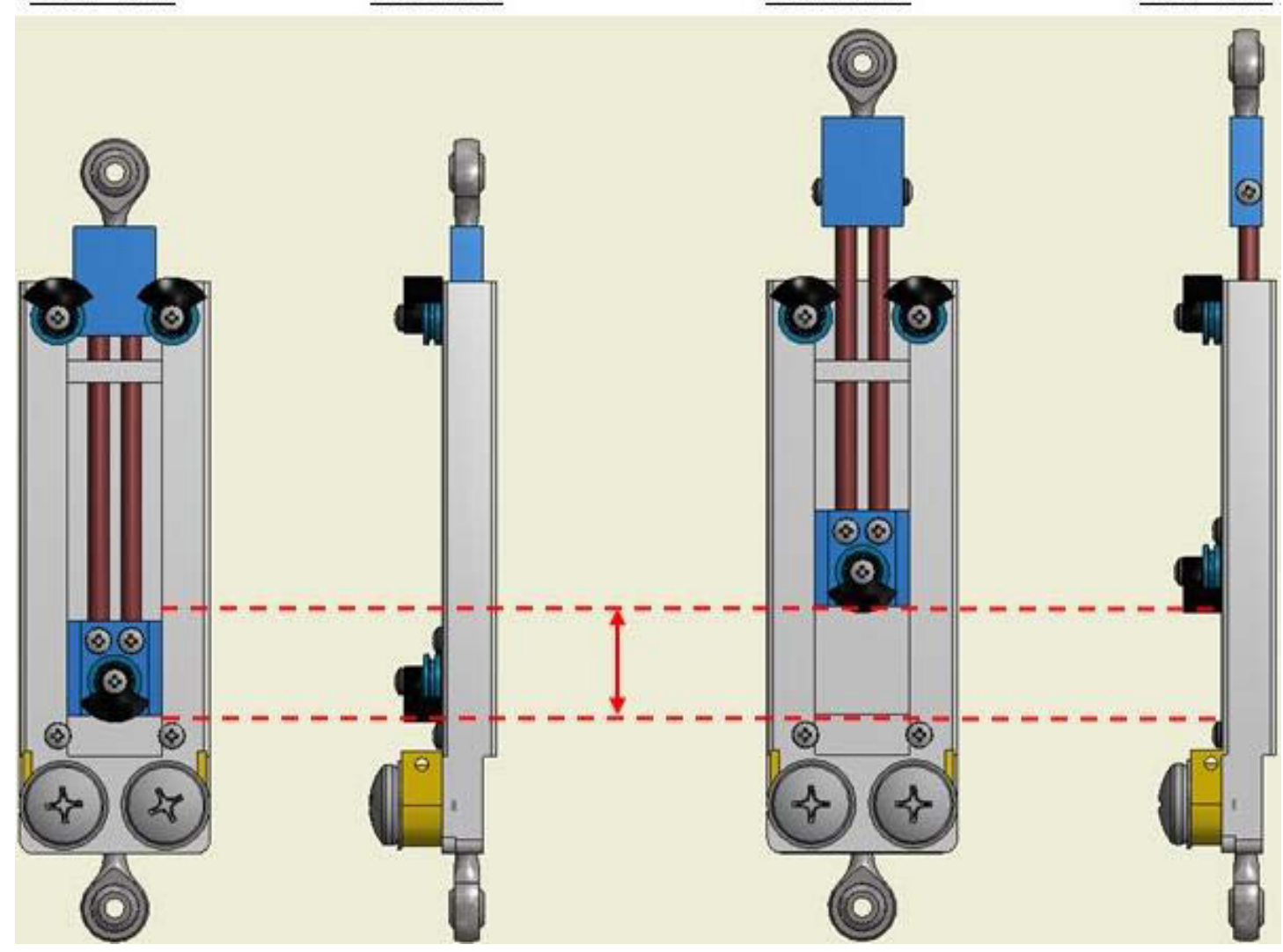

Figure 3-18. The mechanical actuator guides vertical translation of two aluminum rods 


\section{$\underline{\text { Bottom View }}$}

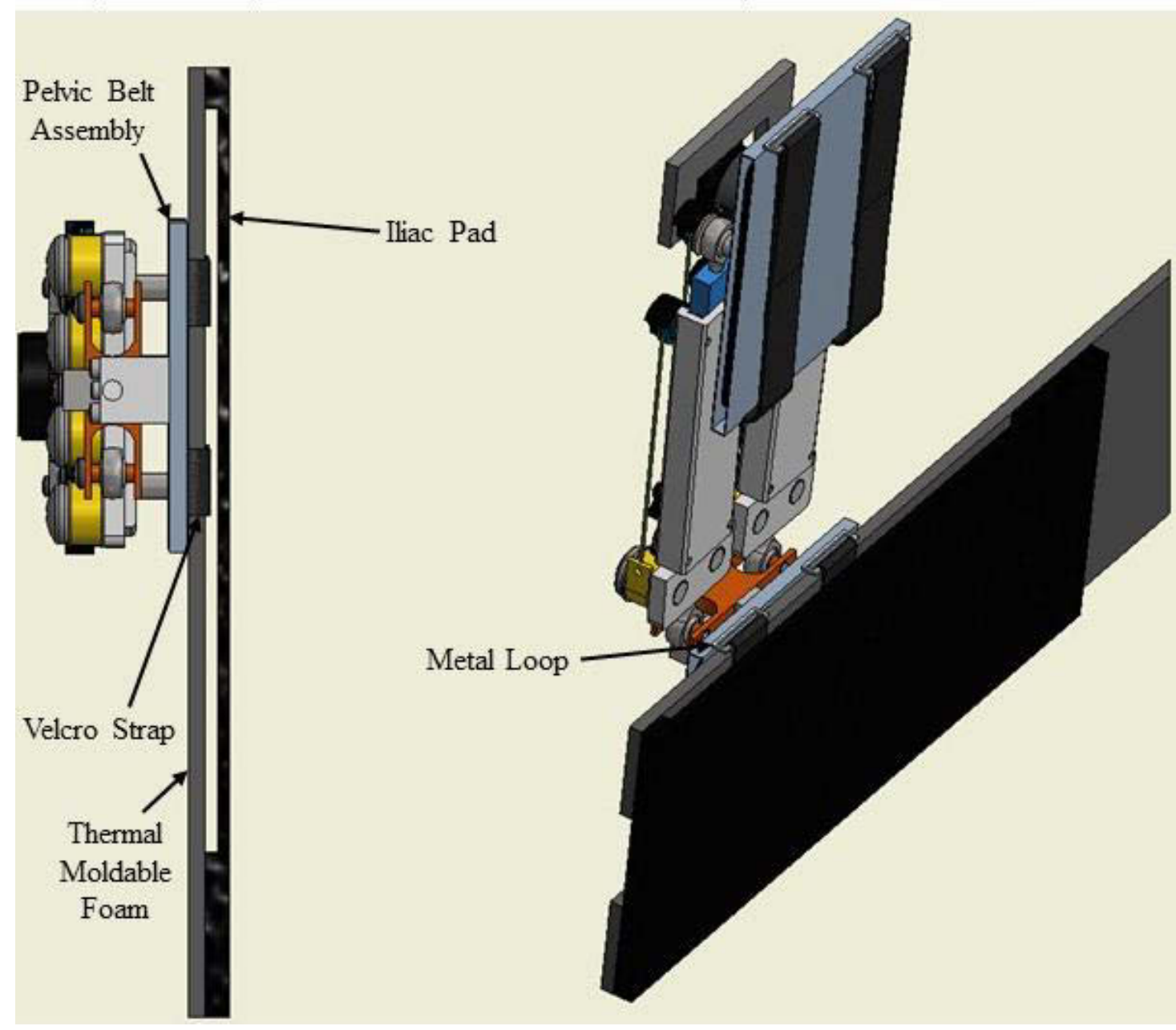

Figure 3-19. The pelvic belt assembly attaches to the pelvic belt similarly to the torso belt assembly's attachment to the torso glove 


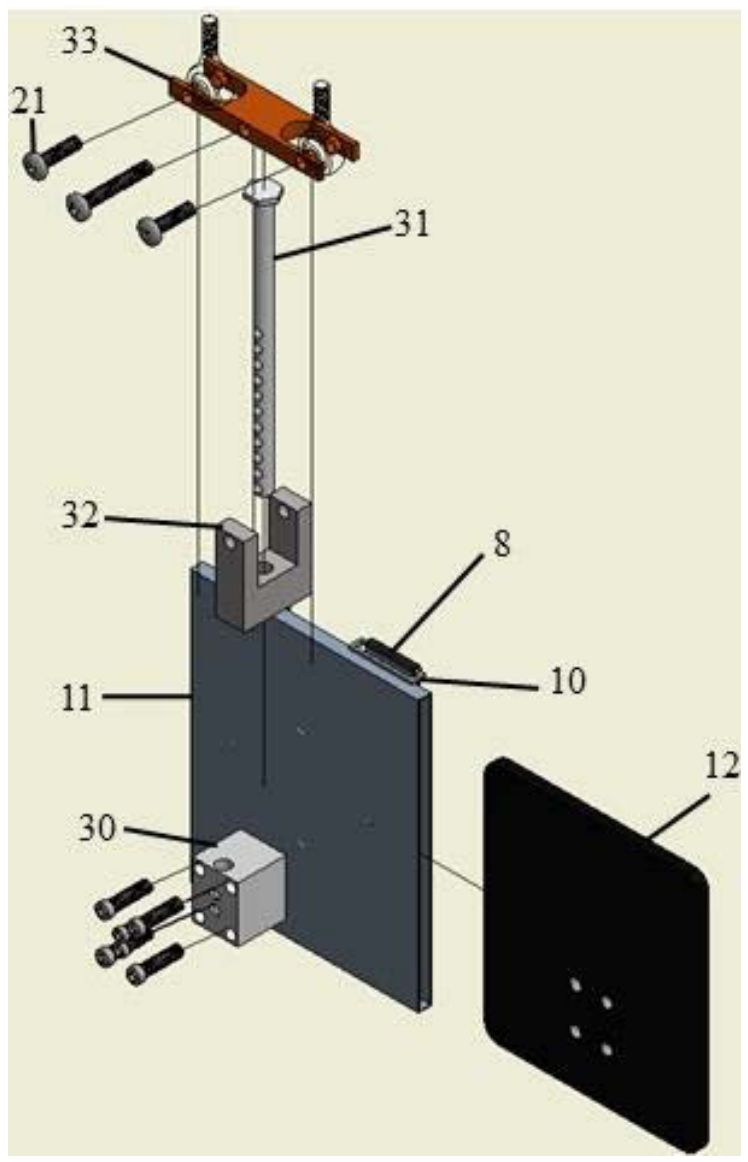

Figure 3-20. The components of the pelvic belt assembly 
assembly through the plastic receiver (30). A rocker (33) was attached to the rod clevis (32) through a bolt (21). The inferior tie rod end (23) on each mechanical actuator attached to the left and right sides of the rocker (33), respectively, through bolts (21). The pelvic belt assembly allowed for rotational axis height adjustment as well as for axial rotation and or flexion or extension movements as shown in Figure 3-21.

Like the DMO2 design where the function of the DFC and MEC components functioned independently of each other, DMO3 was designed similarly. Solutions for the second and third limitations of the DMO2 design as well as detailed descriptions of the DMO3 DFC and MEC components are discussed in the following sections.

\section{DMO3 Distractive Force Component}

The DFC was controlled by the CTS which consisted of cable 1 and cable 2 . Cable 1 connected each CFS to the channel slot nut on each mechanical actuator indirectly by routing around a pulley attached to the superior end of the c-channel that attached to a washer which ultimately connected to cable 2 . Cable 2 extended from the tensioning knob on the torso belt assembly, routed around a pulley on the torso belt assembly, and is then routed around a pulley on the channel slot nut before connecting to a washer. The washer connected cable 1 and cable 2 together. In the loaded state, cable 2 shortened in length when the tensioning knob was turned, and the channel slot nut was pulled upward. When the torso belt assembly traveled vertically to the point at which a force resistance was met that was equal to or greater than the total force resistance of all of the CFSs, the CFSs were extended, and the washer that connected cable 1 and cable 2 traveled downward. When the DFC was engaged, a downward force was transmitted to the pelvic belt through the inferior tie rod ends of each mechanical actuator and an upward force was transmitted to the wearer through the torso belt and torso glove. The torso glove can be thought of as a human suspension system that essentially pulled upward on the wearer's torso as the DFC lifts upward. Assuming that all of the engagement components were secured to the wearer without slipping, a distractive force would be applied to the wearer. This mechanism by which the DFC applies a distractive force to the wearer through the pelvic belt and the torso belt and torso glove's engagement is illustrated in Figure 3-22. Although the image in Figure 3-22 is shown such that both mechanical actuators are the same height, both actuators essentially functioned independent of each other meaning if one actuator needed to lengthen and the other shorten during movement, it would be able to do so. When the DFC was engaged, a downward force was transmitted to the pelvic belt through the inferior tie rod ends of each mechanical actuator, and an upward force was transmitted to the wearer through the torso belt and torso glove. The torso glove can be thought of as a human suspension system that essentially pulled upward on the wearer's torso as the DFC lifts upward. Assuming that all of the engagement components were secured to the wearer without slipping, a distractive force would be applied to the wearer. After assembly of the DFC of DMO3, it was determined that it was too difficult to achieve the initial goal of $300 \mathrm{~N}$ for a DFC goal. Because of this, each constant force spring was set to have a force output of 
Axial Rotation

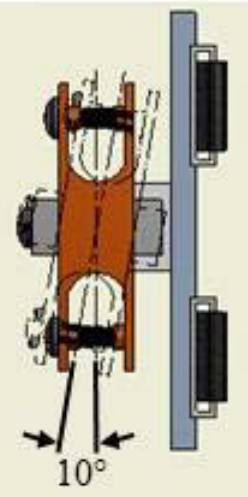

\section{$\underline{\text { RAAdjustment }}$}

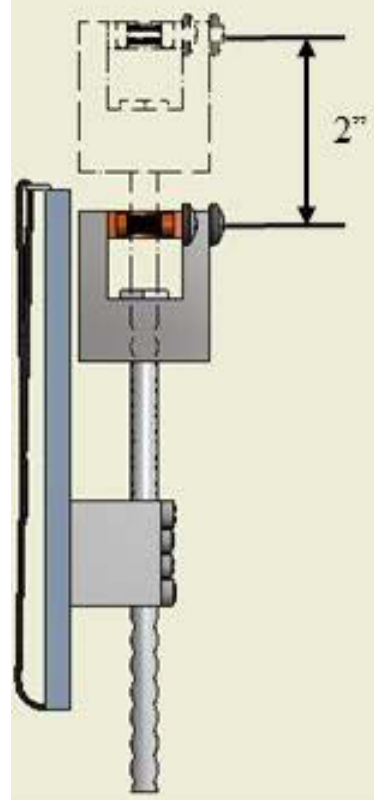

Flexion or Extension

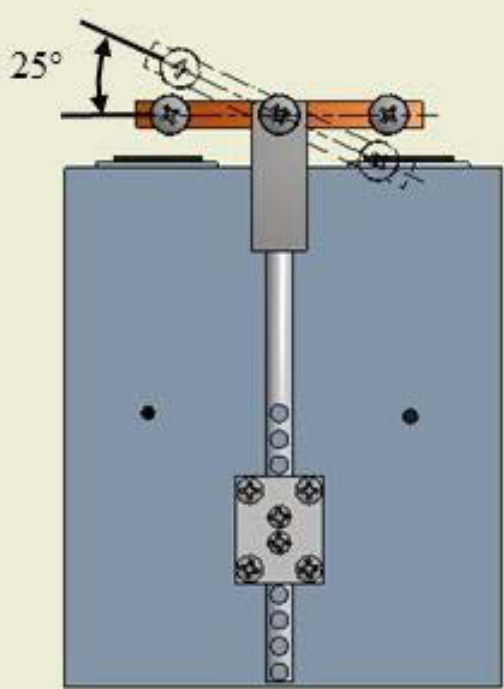

Figure 3-21. The pelvic belt assembly allows for flexion or extension, axial rotation, and vertical rotational axis adjustment 


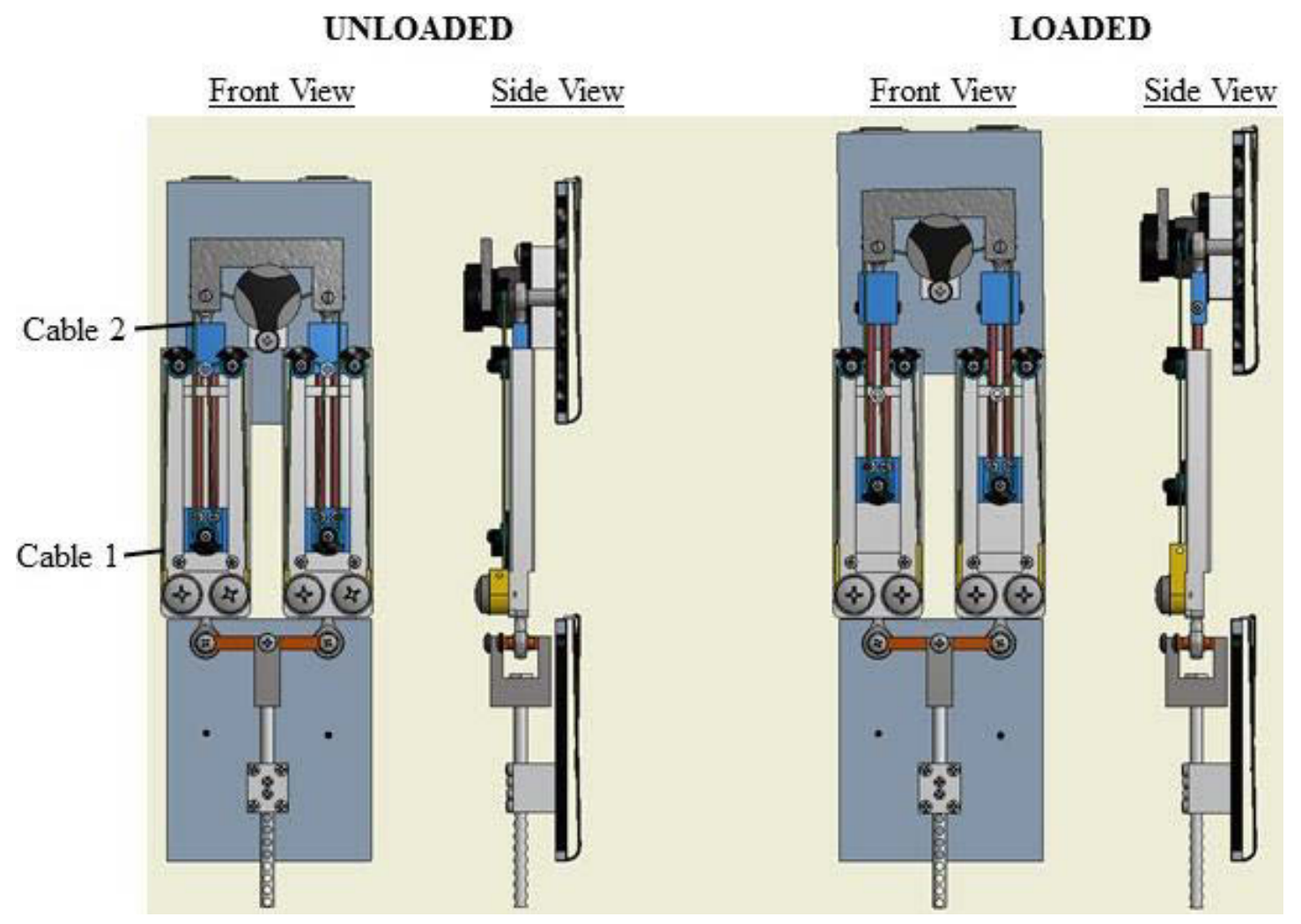

Figure 3-22. The distractive force component provides a distractive force to the wearer in the loaded state 
five pounds. Since each DFC had exactly four constant force springs, the total distractive force output was set to 40 pounds (approximately $180 \mathrm{~N}$ ). If desired, the total force output of the DFC could be altered by switching out constant force springs with different force outputs.

\section{DMO3 Mobility-Enabling Component}

The pelvic belt assembly, as previously mentioned, allowed for axial rotation and/or flexion or extension movements. The superior end of the axis adjustment bar was inserted into a hole on the superior side of the rod clevis, and the posterior side of the rod clevis was securely sitting on the axis adjustment bar with a diameter larger than the hole in the swivel. Therefore, the rod clevis was allowed to rotate freely about the long axis of the axis adjustment bar under simulated axial rotation as shown in Figure 3-23. The rocker was attached to the rod clevis through a screw located at the RA of the DFC. The location of the screw that connected the rocker to the rod clevis served as a pin joint that allowed the user to bend freely in flexion or extension as shown in Figure 3-24. Because the DFC utilized CFSs to apply the input force into the DFC, the mobility-enabling component (MEC) should be able to allow flexion/extension or axial rotation movement without losing much force output from the mechanism. Because of this, the wearer can perform DLAs with minimal resistance to movement and with a distractive force being applied at all times by the DMO.

The inferior end of the axis adjustment bar was anchored to the plastic receiver on the pelvic belt assembly. The RA of the DMO can be located vertically with respect to the pelvic belt by unscrewing the bolts that secured the axis adjustment bar to the plastic receiver and adjusting the vertical location of the axis adjustment bar. The RA of the DMO can be located horizontally by sliding the torso belt assembly and pelvic belt assembly side-to-side on the torso belt and pelvic belt, respectfully.

\section{Protocol for Testing and Evaluating DMO3 in the Laboratory Under Simulated Flexion and Extension}

DMO3 was evaluated in the laboratory similarly to DMO2; however, the testing conditions for the DMO3 prototype were different. The testing conditions for the DMO3 prototype were an upper torso load of $150 \mathrm{~N}$ in upright stance, initiation of flexion and extension, and extended ranges of $25^{\circ}$ of flexion and $10^{\circ}$ of extension. The $150 \mathrm{~N}$ value represented both half of the force that was tested with DMO1 and DMO2 and a number close to the $180 \mathrm{~N}$ maximum force capacity determined to be sufficient for DMO3. To apply these force conditions, the same advanced control strategy methods described in Figure 3-7 were used. The DMO3 prototype was then then mounted on the testing platform, the RA was aligned with the spine, and the advanced testing protocol was rerun to simulate the testing conditions above. 




Figure 3-23. The distractive force component in upright stance and axial rotation 


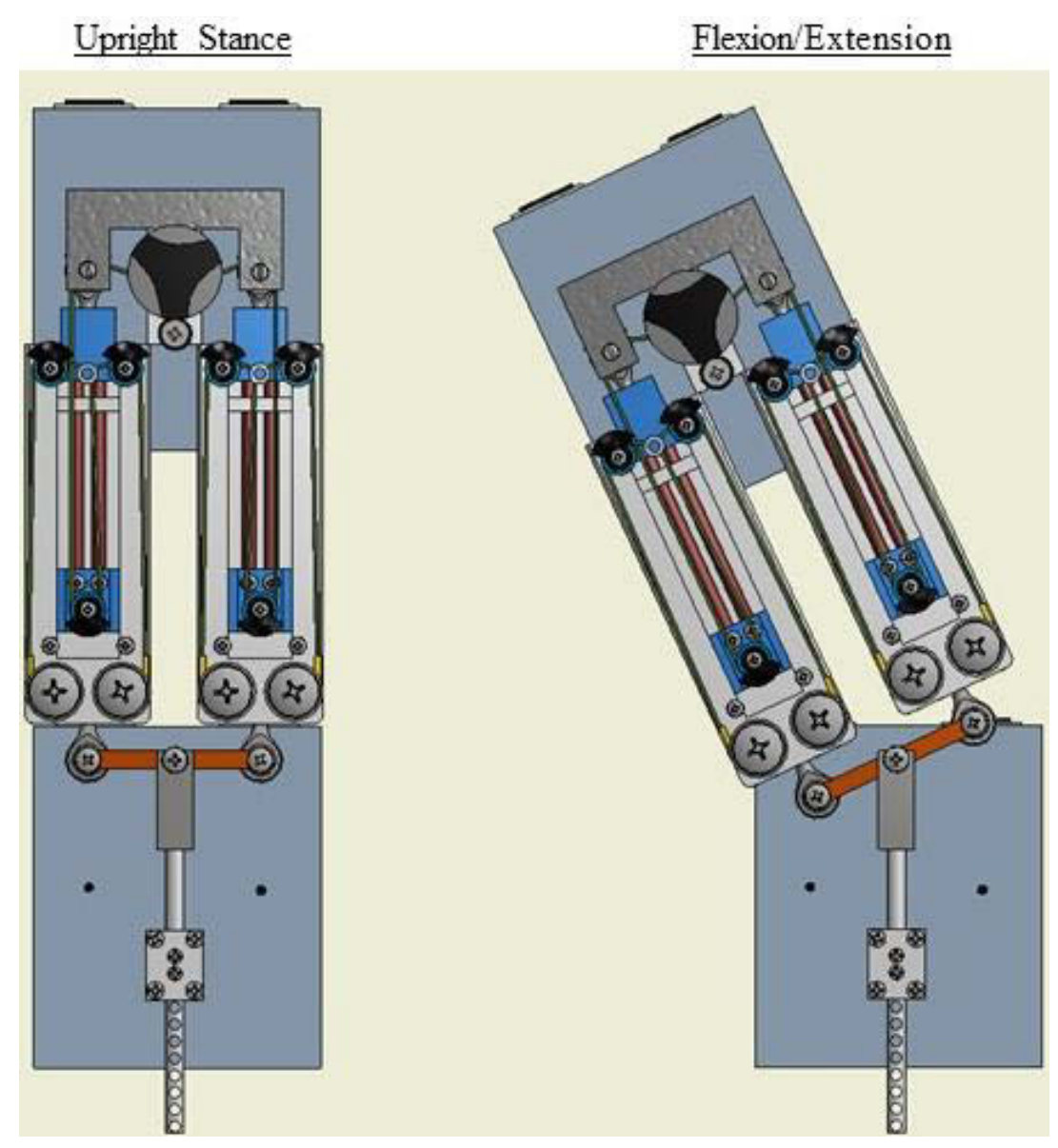

Figure 3-24. The distractive force component in upright stance and in flexion or extension 
The primary outcomes for DMO3 laboratory testing were spinal off-loading (brace load) and bending moment (brace effect) at upright stance and during flexion and extension ranges. Applied loads to the torso-orthosis assembly by the RTP were measured at the ULC and the loads transferred through the lumbar spine were measured at the BLC. The ULC and BLC forces and bending moments (BM) were transformed to the sacral disc plane (SDP) and compared in flexion and extension as previously shown in Figure 3-8a. The difference in the applied load and the transferred load represented the brace load carried by DMO3. This concept is illustrated in Figure 3-8b. Figure 3-8c showing how the difference in the applied moment and the transferred moment was represented the brace effect moment caused by DMO3.

\section{Protocol for Testing and Evaluating DMO3 in the Laboratory Under Simulated Axial Rotation}

The capacity of the DMO3 prototype to undergo simulated axial rotation was tested in the RTP. First, the upper torso was mounted to the ULC like the previous test. This upper torso would later serve as the engagement for the torso glove and the torso belt just as it was in study one under simulated flexion and extension. Next, a rotary turntable was mounted onto the baseplate of the RTP. Then, the pelvic girdle was mounted onto the rotary turntable as shown in Figure 3-25a. With all of the components except for the DMO mounted appropriately in the RTP, the human model could undergo simulated axial rotation as shown in Figure 3-25b by manually rotating the turntable to the left or to the right. Rotating the turntable clockwise simulated left axial twisting, and rotating the turntable counterclockwise simulated right axial twisting. A tensiometer (Esky Digital Scales, USA) with a resolution of $\pm 0.09 \mathrm{~N}(0.02 \mathrm{lbs})$ was attached to the turntable as seen in Figure 3-25c. A measurement was made from the center of the turntable to the location of the tensiometer. This known distance combined with the tensiometer readout ensured that passive rotation did not exceed $0.1 \mathrm{Nm}$.

Figure 3-26a shows the testing assembly previous described with the DMO being appropriately placed on the human torso and pelvic girdle. With the DMO mounted onto the torso model and the pelvic girdle, the turntable was passively turned clockwise and counterclockwise three separate times. During each trial, the turntable was turned until it could not be turned anymore without damaging the DMO as shown in Figure 3-26b. Before the turntable was turned during each trial, a mark (mark 1) was made on the turntable based on the location of a bolt that was normal to the rotary turntable's interface with the pelvic girdle as shown in Figure 3-27a. At the end point of rotation for each direction, another mark (mark 2) was made based on the location of that same bolt as shown in Figure 3-27b. At the completion of each trial, a goniometer was aligned with the center of the rotary turntable and used to measure the angle between mark 1 (before passive rotation) and mark 2 (at the end of passive rotation) as shown in Figure 3-27c. 

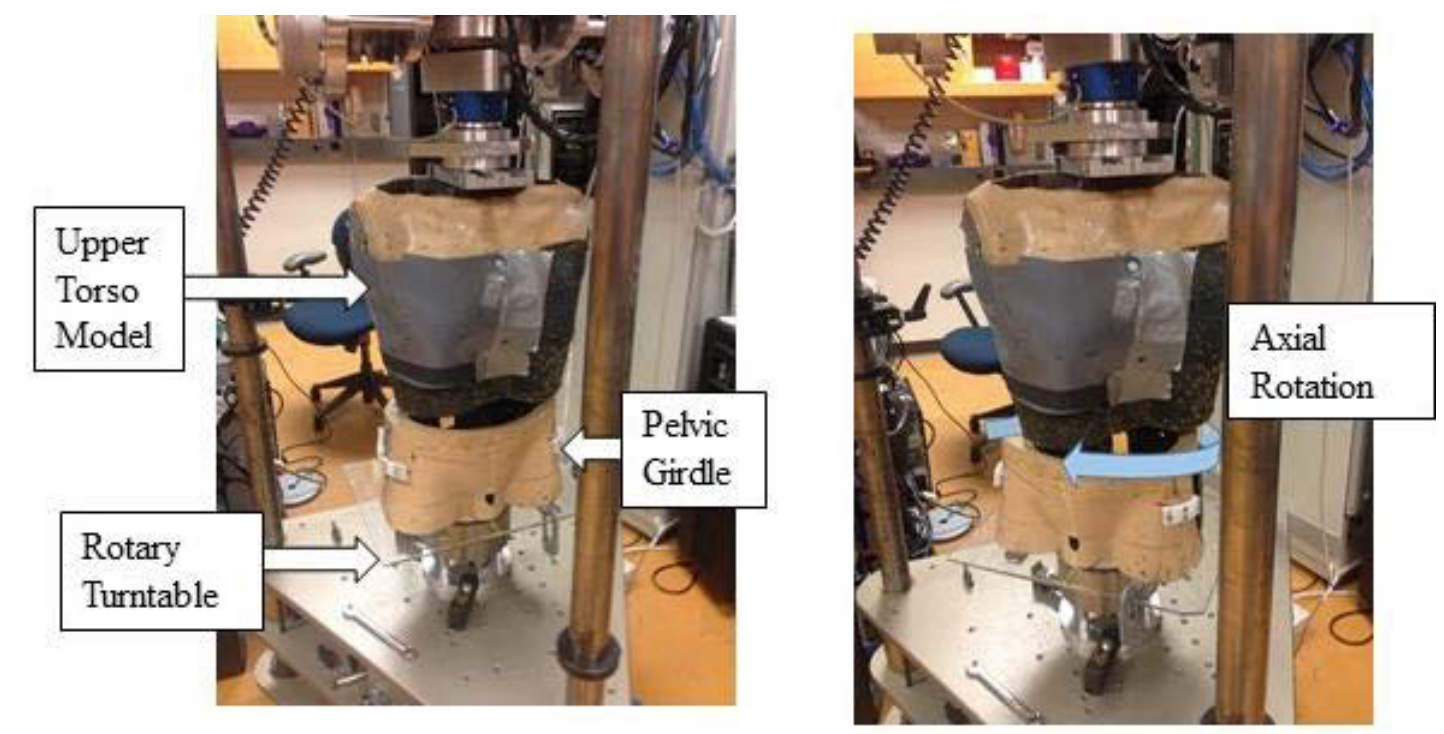

(a)

(b)

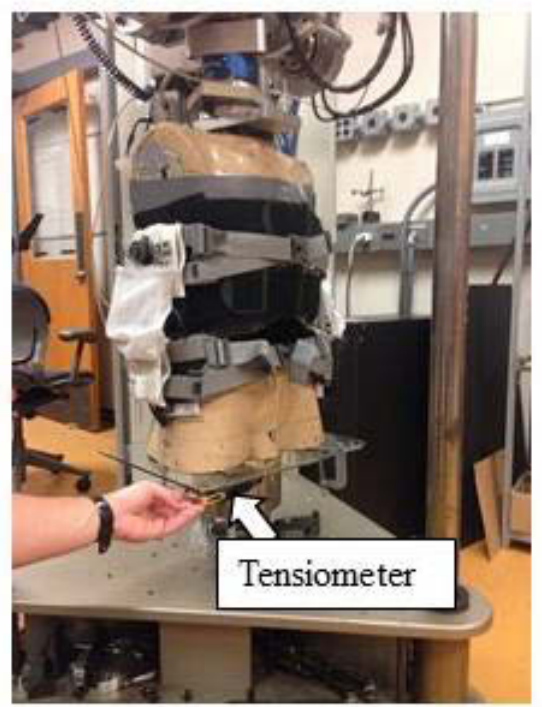

(c)

Figure 3-25. Set-up for testing axial rotation capacity of DMO3 with the human analog torso model mounted on a rotary turntable in the RTP

(a) The upper torso model was superiorly mounted to the ULC, a rotary turntable was inferiorly mounted to the base plate of the RTP, and the pelvic girdle was inferiorly mounted to the rotary turntable.

(b) Left axial twisting is simulated by passively rotating the turntable clockwise

(c) A tensiometer was used to ensure passive rotation did not exceed $0.1 \mathrm{Nm}$ 


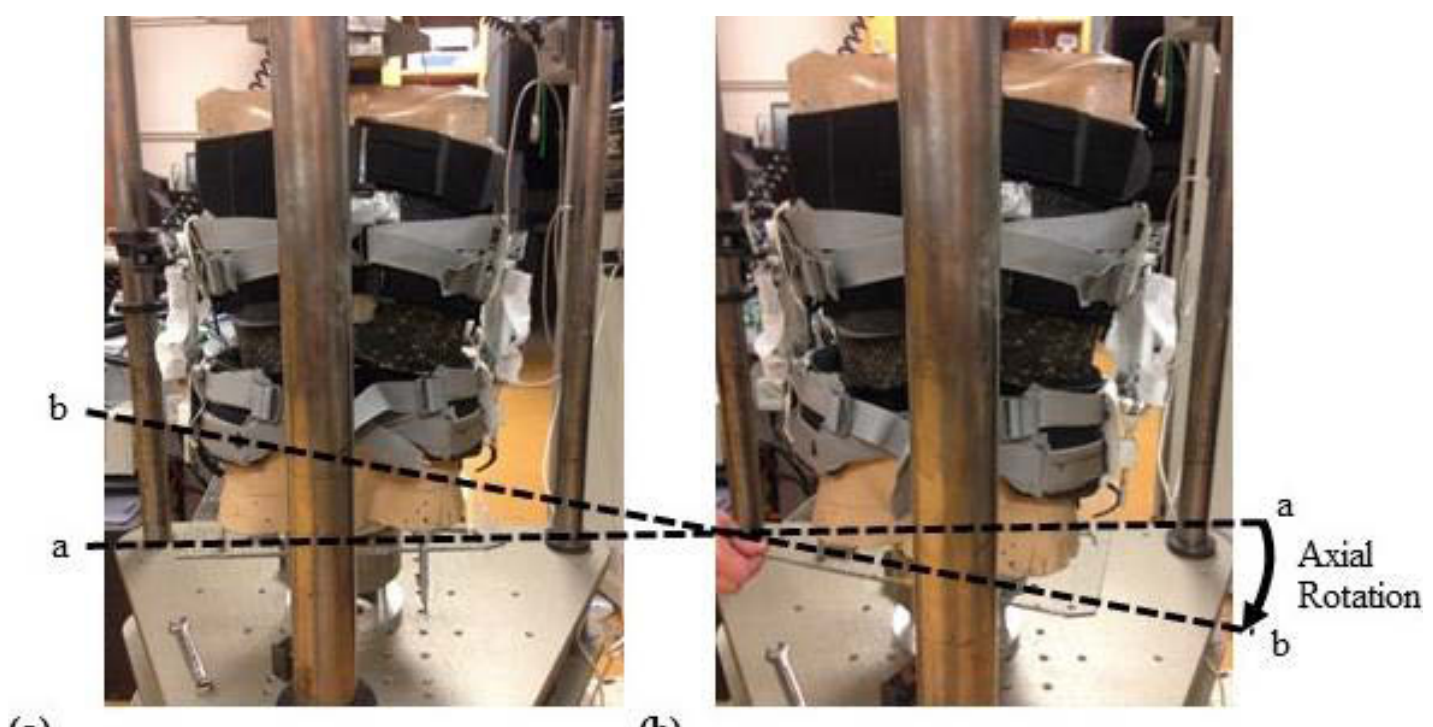

(a)

(b)

Figure 3-26. DMO3 on the human analog torso model in the RTP for axial rotation capacity testing

(a) The DMO3 prototype mounted on the upper torso model and pelvic girdle in simulated upright stance

(b) The DMO3 prototype in simulated left axial twisting 


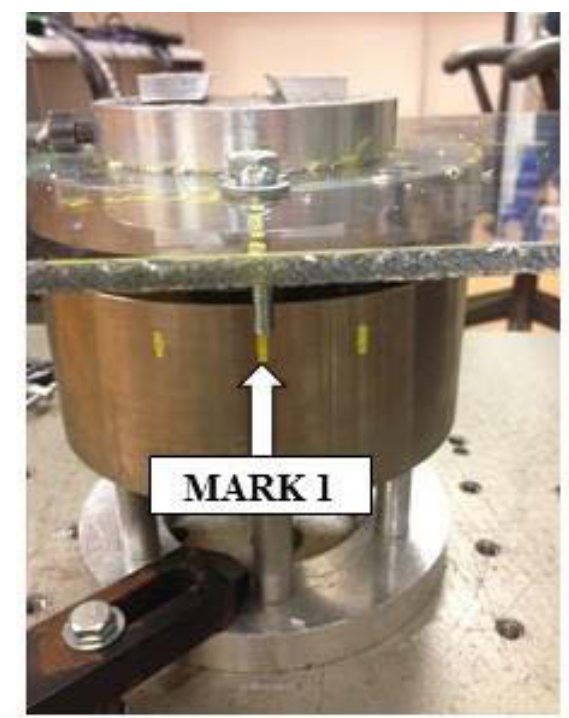

(a)

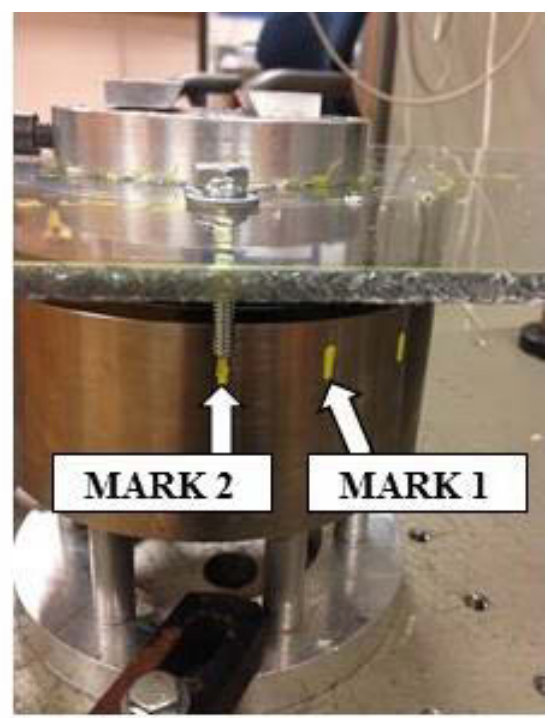

(b)

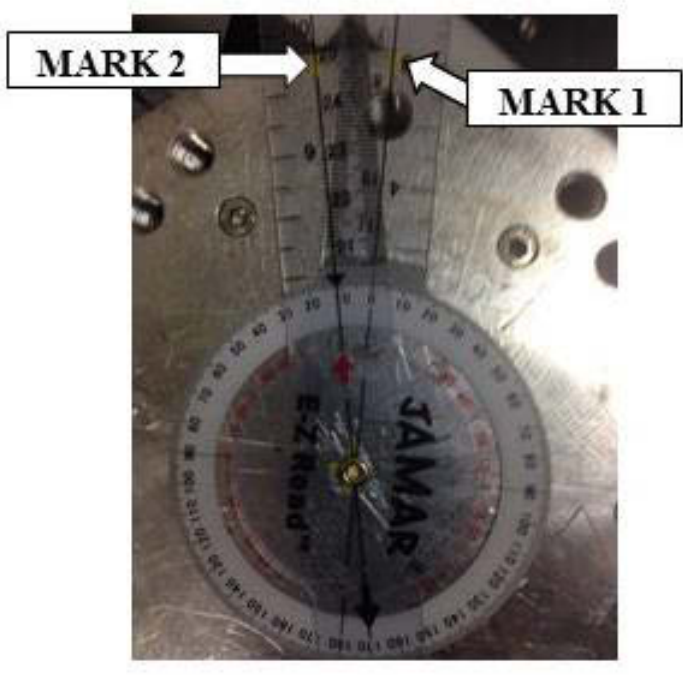

(c)

Figure 3-27. Measurement method for determining axial rotation capacity of DMO3

(a) Mark 1 was made on the rotary turntable before passively moving the turntable in either direction

(b) Mark 2 was made on the rotary turntable after passively moving the turntable in either direction

(c) After mark 1 and mark 2 were made on the rotary turntable at the completion of each trial, a goniometer measured the angle between mark 1 and mark 2 to determine the amount of axial rotation DMO3 allowed under simulated axial twisting 


\section{Clinical Testing of DMO3}

\section{Case 1}

Patient 1 was a female (age 49) suffering with low back pain down to the right lower extremity, mainly the buttock and thigh area. This patient had been diagnosed with lumbar degenerative disc disease at the L5-S1 level. She had been working with PT, and was recommended by her physical therapist to alternate with some dates at a pool to exercise. She said that slowly but steadily, she kept improving; however, the pain down to the right thigh had not really been resolved. Her back pain still persisted. The intensity depended on what she did during the day, but she said that she could tolerate a pool exercise program. She said that really she was progressing with the pool therapy, but the pool she has at home has been closed for the rest of the year. Anything that involved sitting for over 30 minutes or standing over an hour tended to flare up her symptoms.

\section{Case 2}

Patient 2 was a female (age 44) suffering with on-and-off low back pain that had recently started going to the right lateral hip and thigh area. This patient had been diagnosed with lumbar degenerative disc disease at the L4-L5 and L5-S1 levels with probable discogenic pain. Also, she had been diagnosed with lumbopelvic muscle imbalances and a weak core. Previously, this patient had been prescribed pain medication.

\section{Standard-of-Care for LBP Patients}

Typical standard-of-care for a patient that was suffering from low back pain usually begins when the patient came to Dr. Rivera's office looking for pain-relief solutions. Dr. Rivera would sometimes prescribe anti-inflammatory and muscle relaxer medication, and he would usually suggest that the patient undergo PT which was overseen by a physical therapist at any Campbell Clinic Orthopaedics location. The frequency for which the patient was recommended for PT treatment sessions depended on the patient's schedule; however, most patients typically came to PT 2-3 times per week. Before PT treatments began, the patient completed the mODI issued by the physical therapist. During each treatment session, the patient was instructed by the physical therapist to perform lumbar spine core strengthening (LSCS) exercises, and a numeric pain rating scale (NPRS) score was taken. Each treatment session usually lasted about 3040 minutes. After 6 weeks of PT treatment, Dr. Rivera evaluated the prescribed treatment's effectiveness based on if the patient says that both his or her low back pain had decreased and if he or she was able to function more effectively during his or her daily living activities. If Dr. Rivera determined that medication and PT treatments were not effective, an x-ray was taken to determine if the patient has a spinal pathology that could potentially be causing low back pain. 


\section{DMO3 Clinical Testing Protocol}

The DMO3 prototype was worn for between 20-30 minutes at Campbell Clinic Orthopaedics during patient's standard-of-care PT treatment sessions. The study was performed in collaboration with Dr. Carlos Rivera who specializes in Physical Medicine $\&$ Rehabilitation. So far, the study has included 2 patients, and the duration of the study for each patient was two to three weeks and occurred throughout seven standard-of-care visits for each patient recruited. Any subsequent visits were optional. For example, if the patient was really enjoying his or her treatment sessions with DMO3, he or she could ask to continue treatment with DMO3 in subsequent visits after the seven standard-of-care visits had been completed.

The first visit was one in which the patient had a follow-up visit to Dr. Rivera for a clinical evaluation, and it had been determined that the typical standard-of care treatment was not working for the patient. During this visit, informed consent was obtained, and a standard-of-care $\mathrm{x}$-ray (x ray 1) was performed on the patient while wearing the pelvic belt of the DMO. This standard-of-care x-ray (x-ray 1) during the first visit was done in order to confirm and record a measure of distance across the degenerative lumbar level(s) as well as to determine the location of the L3-L4 lumbar intervertebral disc level with respect to placement of DMO3's pelvic belt. If a patient had degenerative disc disease, for example, at L4-L5 and L5-S1, the disc height was measured at those two levels as well as at L3-L4 as it was recommended to measure the adjacent level(s) as well. The L3-L4 disc location was used to approximately locate where the RA of DMO3 would be placed for each patient. During the second through seventh visits, each patient came to Campbell Clinic Orthopaedics PT for his or her standard-of-care lumbar spine core strengthening (LSCS) PT treatment sessions (as instructed by his or her PT) while wearing DMO3. During the seventh visit, each patient also completed the mODI, underwent an X-ray (x-ray 2) without wearing the DMO to further examine any possible changes in the spinal pathology, and was rated for his or her overall satisfaction of treatment with DMO3. After the seventh visit, the patient was allowed to continue standard-of-care treatment with the DMO if he or she has felt a decrease in pain and wished to continue treatment with the DMO. Patients who did not return for follow-up visits were withdrawn from the study. A summary of the clinical protocol can be seen in Figure 3-28.

Patient risk was minimized as much as possible in this study. There was always a possible risk of loss of confidentiality during a chart review; however, this occurrence was very rare. X-ray 1 (standard X-ray) and x-ray 2 (additional research X-ray) were done using the XGEO GC80 Station Samsung. With its advanced technology, Samsung's thin film transistor-based flat panel detectors built-in advanced low dose amorphous silicon sensor delivered maximized image quality, immediate results and patient safety. The total additional amount of radiation that each patient received by participating in this study was about $0.3 \mathrm{mSv}$ or $30 \mathrm{mrem}$, and was approximately equivalent to a uniform whole body exposure of 37 days of exposure to natural background radiation. This use involved minimal risk and was necessary to obtain the research information desired. Figure 3-29 illustrates the method by which lumbar disc heights were measured. It was assumed that 

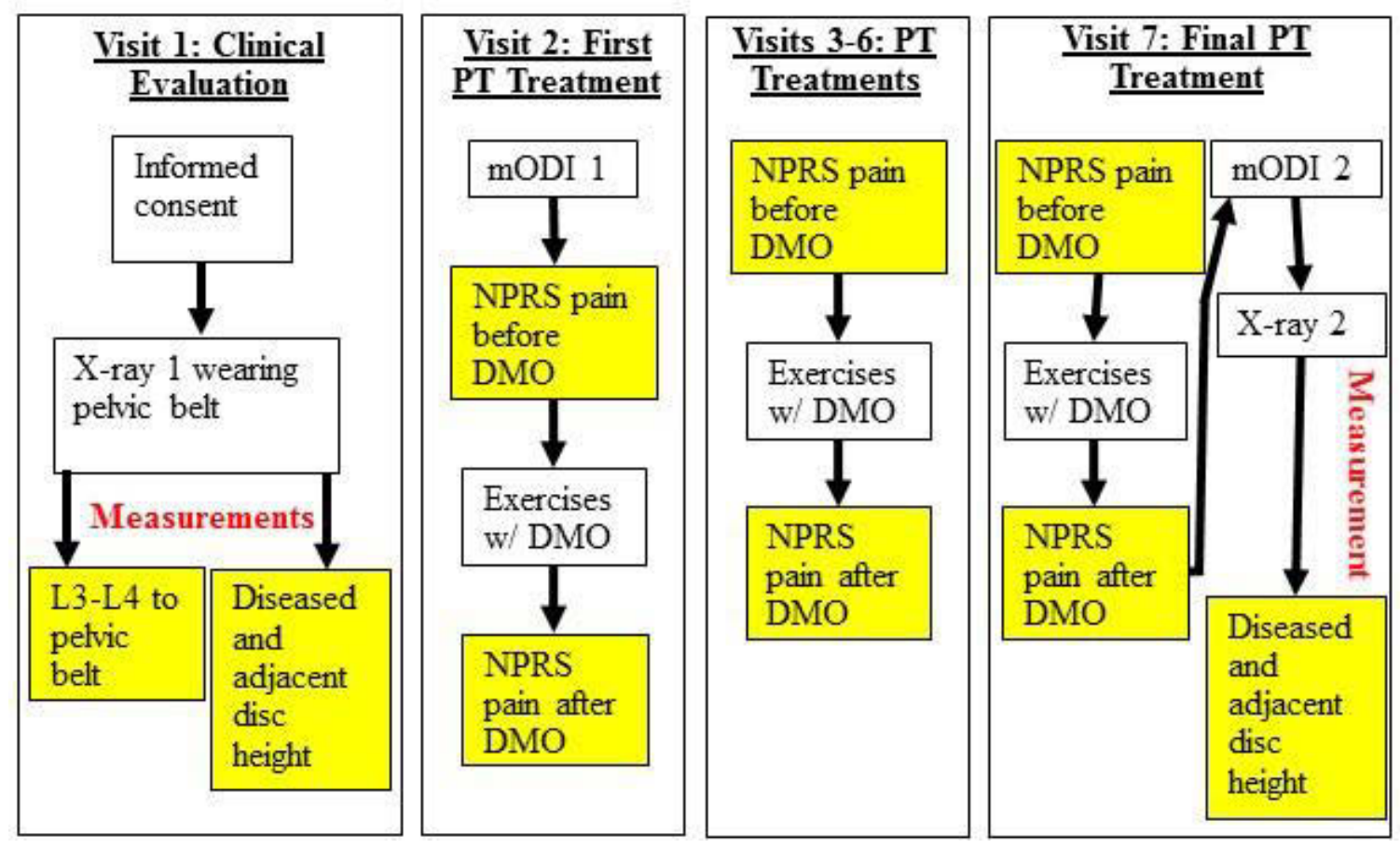

Figure 3-28. A summary of the clinical protocol for testing DMO3 on human subjects at each visit 

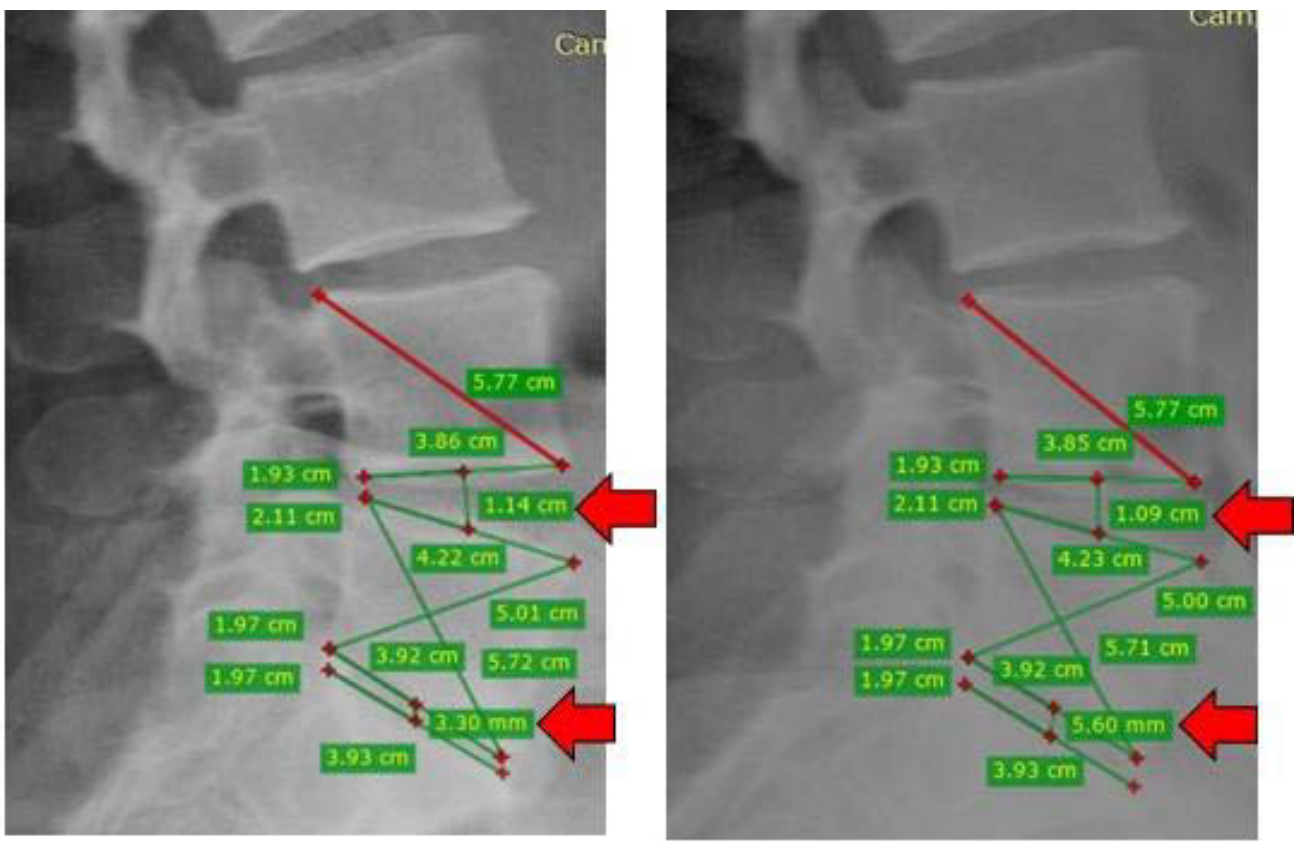

(a)

(b)

Figure 3-29. X-ray measurement technique for clinical testing of DMO3

(a) X-ray 1 before visit 1

(b) X-ray 2 after last treatment visit 
each patient stood in the same spot each time before being radiographed. On the x-ray 1 image, a diagonal measurement was made across a vertebral body. The same measurement was made on $\mathrm{x}$-ray 2 . This measurement served as a calibration between the lengths of the two radiographic images. The corners of each vertebrae served as known points to define endplate orientation. The midpoint of each endplate was found, and a measurement was made connecting the midpoints of two endplates across a lumbar disc. That measurement served as the lumbar disc height that was recorded. It was possible that the DMO could cause discomfort. The time that each patient wore the DMO was minimized while still obtaining meaningful results.

Inclusion and exclusion criteria. So far, two patients who regularly seek conservative treatment at Campbell Clinic Orthopaedics- Spine Center have been recruited and have begun to participate in this study. These patients, and subsequent patients in the study, were between the ages of 25 and 55 years of age and suffer from degenerative disc disease or lumbar foraminal stenosis with associated low back pain and/or leg pain. These patients had previously undergone the standard-of-care treatment without DMO3, as previously described, and it was determined that the treatment was unsuccessful or not as successful as hoped. Therefore, each patient acted as his or her own control.

Patients who had previously undergone spinal fusion surgery were excluded from the study. Also, patients that suffer from any type of scoliosis did not participate in the study. If the patient was younger than 25 or older than 55 years of age, he or she was excluded from the study. Finally, any patients with non-specific low back pain or any kind of spinal implant were excluded from the study.

Outcome measures. A NPRS score was recorded at every visit to determine if there was a decrease in pain over the time in which treatment with the DMO had been administered. During the second through seventh visits, a NPRS score was taken without wearing the DMO and while wearing the DMO. At each treatment visit, a 30\% decrease or a 2-point decrease in NPRS score was determined to be clinically significant [39]. A two-tailed paired t-test (alpha $=0.05)$ was done for NPRS scores before and after each treatment for each patient to determine DMO3's immediate effects on pain relief. The mODI scores for each patient were compared before the first treatment visit and after the final treatment visit with the DMO to determine if there is an improvement in the quality of life of the patient after treatment with DMO3. The mODI scores are calculated by adding up the total score of the circled answers, dividing that total score by the 50 total points available, and multiplying the result by $100 \%$. The mODI score differences were determined to be clinically significant if a decrease of 10 percentage points was seen between the first and final treatment visit [40]. Lumbar measurements from the x-ray across the diseased and adjacent levels were compared before (Visit 1) and after (Visit 7) treatment with the DMO to determine if there was an increase in lumbar disc height. The resolution of the XGEO GC80 Station Samsung was $0.14 \mathrm{~mm}$. All x-ray measurements were rounded to the nearest half of a millimeter. In the future, this measure could be used to determine if there is a correlation between a decrease in NPRS score (between the first 
and final treatment visits) and an increase in lumbar disc height. Overall patient satisfaction was used to determine the functional benefits of wearing the DMO. 


\section{CHAPTER 4. RESULTS*}

\section{Laboratory Testing of DMO2 and DMO3 Under Simulated Flexion and Extension}

\section{DMO2 Spinal Off-Loading Analysis}

The off-loading capacity of the modified (DMO2) and original (DMO1) prototypes is shown in Figure 4-1 for the 300N upper body weight loading condition in the upright stance configuration and through extended ranges of $25^{\circ}$ flexion and $10^{\circ}$ of extension [16, 33, 41]. The percentage of the applied load carried by each orthosis as well as the loads transferred to the spine are given in Table 4-1 along with the loads carried by the orthoses and the load transferred through the lumbar spine. The load values for DMO1 were determined in a previous study under similar test conditions [16]. At end range flexion, DMO2 supported almost all of the applied load (i.e., 99\%) compared to $59 \%$ for DMO1. At the end range extension, all of the applied load was supported by DMO2 and the spine was placed under slight traction (102\%) compared to $85 \%$ support for DMO1.

\section{DMO2 Bending Moment Analysis}

The sagittal bending moment versus angular displacement response of the modified (DMO2) and original (DMO1) prototypes is shown in Figure 4-2 for the 300N upper body weight loading condition in the upright stance configuration and through extended ranges of $25^{\circ}$ flexion and $10^{\circ}$ of extension [16, 33, 41]. End range moment values for both DMO1 and DMO2 are listed in Table 4-2. The applied moment required to reach $25^{\circ}$ flexion was $21.7 \mathrm{Nm}$ for DMO2 and $32.4 \mathrm{Nm}$ for DMO1. A similar reduction in the bending moment was required to reach $10^{\circ}$ of extension by DMO2 (10.9 $\mathrm{Nm})$ compared to DMO1 $(15.0 \mathrm{Nm})$. The moment buildup of each orthosis, referred to as the brace effect, is also listed in Table 4-2. At end range flexion, only $6.6 \mathrm{Nm}$ of sagittal moment was required for DMO2 compared to $18.6 \mathrm{Nm}$ for DMO1. At end range extension, the brace effect of DMO2 was 4.4 Nm compared to 15.0 $\mathrm{Nm}$ for DMO1. The moment buildup for DMO2 was 27\% less than that of DMO1 (i.e., 30\% compared 57\%) at end range flexion and $60 \%$ less at end range extension (i.e. $40 \%$ compared to $100 \%$ ).

\footnotetext{
* Portions reprinted with permission from D. DiAngelo and D. Hillyard, "A novel distractive and mobility-enabling lumbar spinal orthosis," Journal of Rehabilitation and Assistive Technologies Engineering, vol. 3, pp. 1-10, 2016.
} 

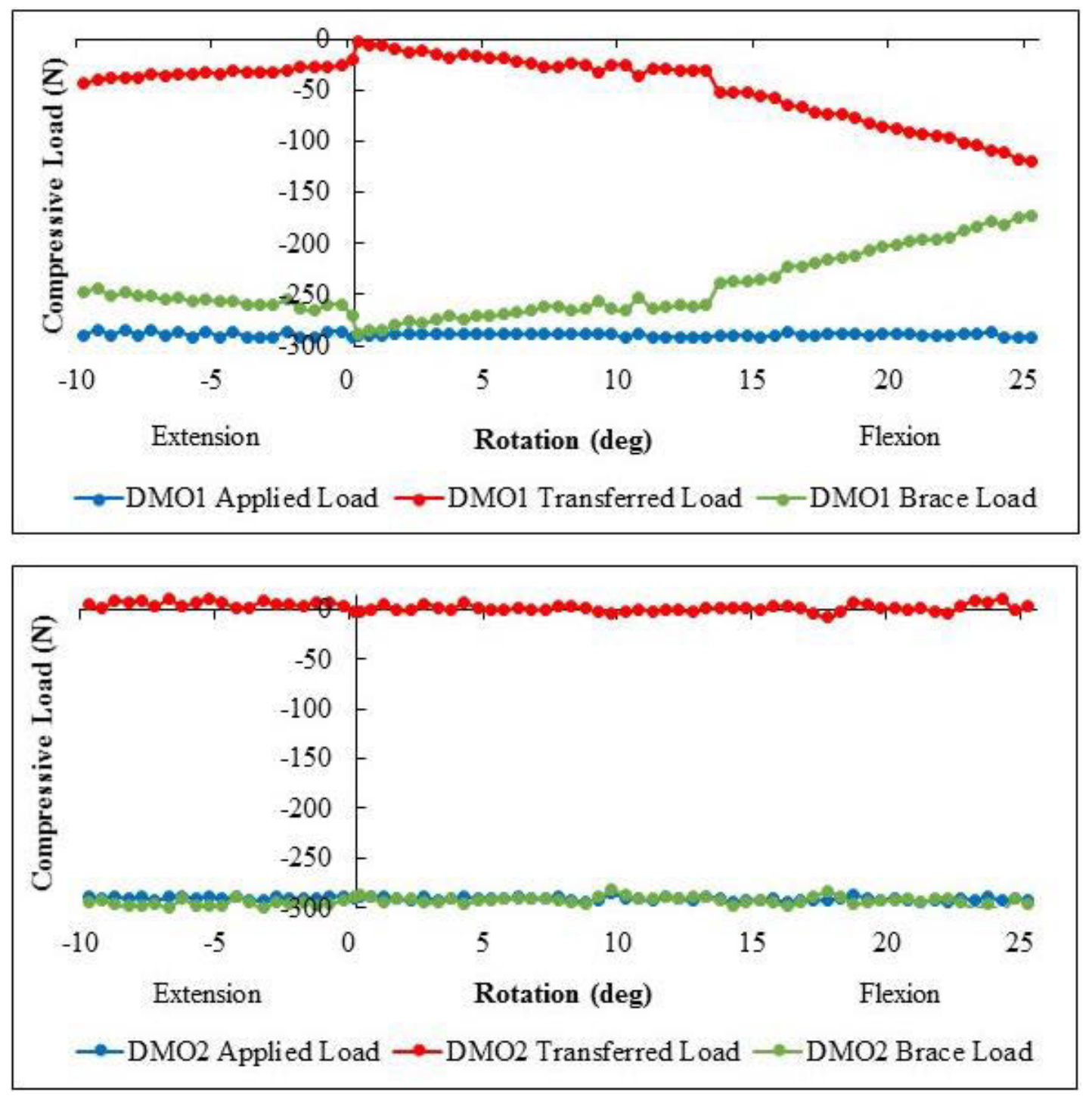

Figure 4-1. Spinal off-loading of DMO2 (bottom) compared to DMO1 (top) in upright stance and throughout extended ranges of flexion and extension 
Table 4-1. Comparison of DMO1 and DMO2 compressive load values at upright stance and at end ranges of motion

\begin{tabular}{lcccc}
\hline $\begin{array}{c}\text { Degrees of } \\
\text { Rotation }\end{array}$ & $\begin{array}{c}\text { Applied } \\
\text { Load (N) }\end{array}$ & $\begin{array}{c}\text { Transferred } \\
\text { Load (N) }\end{array}$ & $\begin{array}{c}\text { Brace } \\
\text { Load (N) }\end{array}$ & $\begin{array}{c}\text { Brace Load as a } \\
\text { Percentage of } \\
\text { Applied Load (\%) }\end{array}$ \\
\hline At $10^{\circ}$ Extension & $290 / 288$ & $43 /-5$ & $247 / 293$ & $85 / 102$ \\
At $0^{\circ}$ & $300 / 288$ & $0 / 6$ & $300 / 282$ & $100 / 98$ \\
At $25^{\circ}$ Flexion & $291 / 293$ & $119 / 3$ & $172 / 290$ & $59 / 99$ \\
\hline
\end{tabular}

DMO1/DMO2 

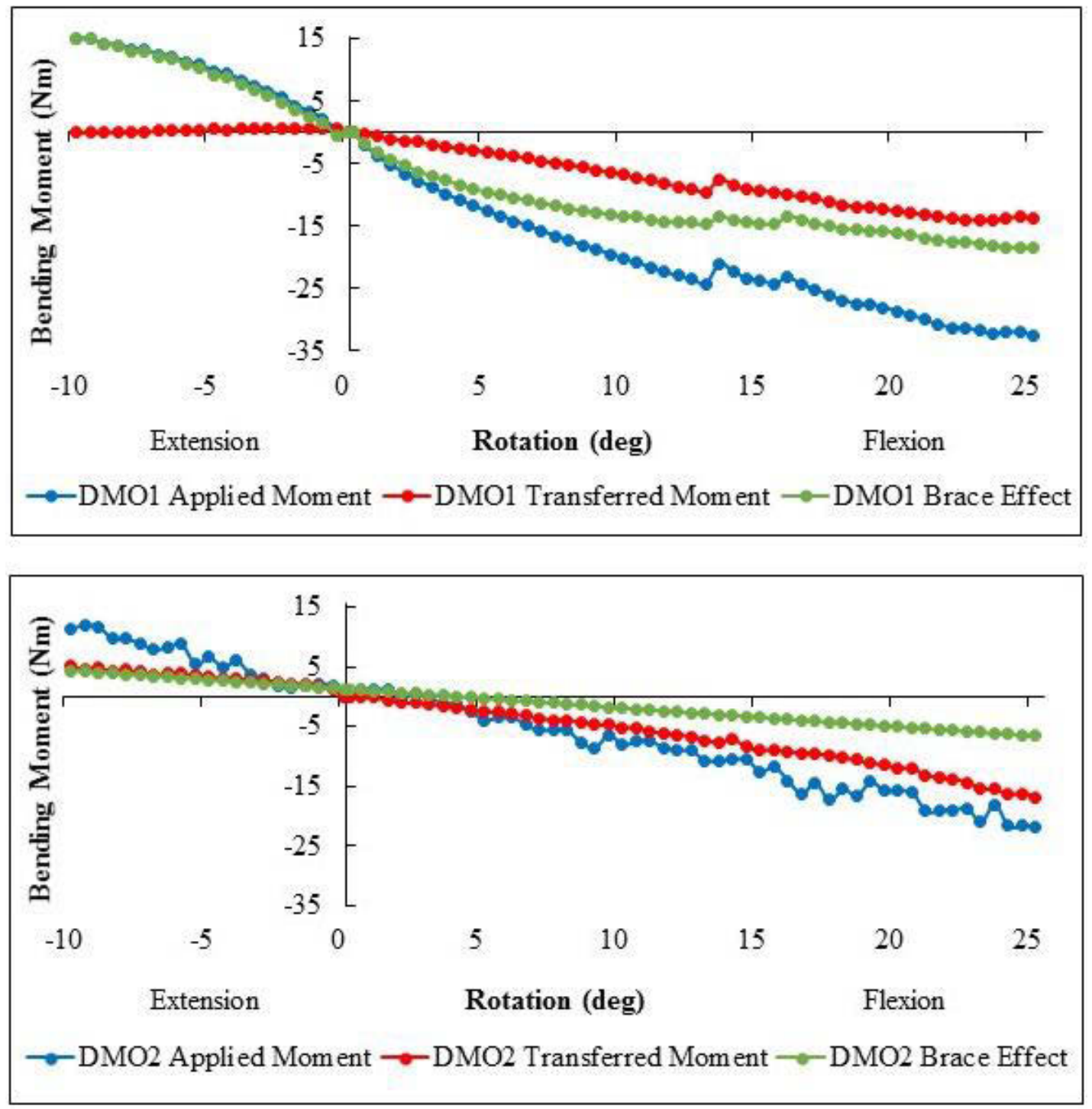

Figure 4-2. Resistance to bending of DMO2 (bottom) compared to DMO1 (top) in upright stance and throughout extended ranges of flexion and extension 
Table 4-2. Comparison of DMO1 and DMO2 bending moment values at end ranges of motion

\begin{tabular}{lcccc}
\hline $\begin{array}{c}\text { Degrees of } \\
\text { Rotation }\end{array}$ & $\begin{array}{c}\text { Applied } \\
\text { Moment } \\
(\mathbf{N m})\end{array}$ & $\begin{array}{c}\text { Transferred } \\
\text { Moment } \\
(\mathbf{N m})\end{array}$ & $\begin{array}{c}\text { Brace } \\
\text { Effect } \\
\mathbf{( N m )}\end{array}$ & $\begin{array}{c}\text { Brace Effect as a } \\
\text { Percentage of } \\
\text { Applied Moment (\%) }\end{array}$ \\
\hline At $10^{\circ}$ Extension & $15 / 10.9$ & $0 / 6.5$ & $15 / 4.4$ & $100 / 40$ \\
At $25^{\circ}$ Flexion & $32.4 / 21.7$ & $13.8 / 15.1$ & $18.6 / 6.6$ & $57 / 30$ \\
\hline
\end{tabular}

DMO1/DMO2 


\section{DMO3 Spinal Off-Loading Analysis}

The off-loading capacity of DMO3 is shown in Figure 4-3 for the $150 \mathrm{~N}$ upper body weight loading condition in the upright stance configuration and through extended ranges of $25^{\circ}$ flexion and $10^{\circ}$ of extension. The percentage of the applied load carried by the orthosis is given in Table 4-3 along with the loads carried by the orthoses and the loads transferred through the lumbar spine. At end range flexion, DMO3 supported all of the applied load and the spine was placed in slight traction (104\%). At end range extension, almost all of the applied load was supported by DMO3 (97\%). In upright stance, DMO3 supported all of the applied load (100\%).

\section{DMO3 Bending Moment Analysis}

The sagittal bending moment versus angular displacement response of the orthosis is shown in Figure 4-4 for the $150 \mathrm{~N}$ upper body weight loading condition in the upright stance configuration and through extended ranges of $25^{\circ}$ flexion and $10^{\circ}$ of extension. End range moment values for DMO3 are listed in Table 4-4. The applied moment required to reach $25^{\circ}$ flexion was $4.1 \mathrm{Nm}$ of which DMO3 contributed none of the bending moment $(0 \%)$. The bending moment required to reach $10^{\circ}$ of extension was 6.6 $\mathrm{Nm}$ of which DMO3 contributed a little more than half of the bending moment $(67 \%)$.

\section{Laboratory Testing of DMO3 Under Simulated Axial Rotation}

The results for the three trials for each direction of rotation of the rotary turntable with DMO3 mounted in the RTP can be seen in Table 4-5. For both directions of passive rotation of the turntable, DMO3 was able to achieve $10^{\circ}$ of axial rotation as measured by the methods previously mentioned. Values were rounded to the nearest whole number.

\section{Clinical Testing of DMO3}

Patient 1 completed six treatment sessions with DMO3, and patient 2 completed four treatment sessions with DMO3. The results for the pain scores of patient 1 and patient 2 before and after wearing DMO3 at their PT treatment sessions can be seen in Figure 4-5 and Figure 4-6, respectfully. For Figure 4-5 and Figure 4-6, the dotted lines represent a clinically relevant decrease in pain [39]. For patient 1, DMO3 completely removed all pain from the patient while wearing the DMO3 and after wearing DMO3 for the remainder of the day at five of the six treatment visits. DMO3 provided a statistically significant $(\mathrm{p}<0.001)$ immediate reduction in pain for patient 1 for the six treatment visits. Similarly, DMO3 completely removed all pain from patient 2 while wearing DMO3 and after wearing DMO3 for the remainder of the day for the four completed treatment sessions. DMO3 provided a statistically significant $(\mathrm{p}=0.005)$ immediate reduction in pain for patient 2 for the four treatment visits completed. Figure 4-7 and 


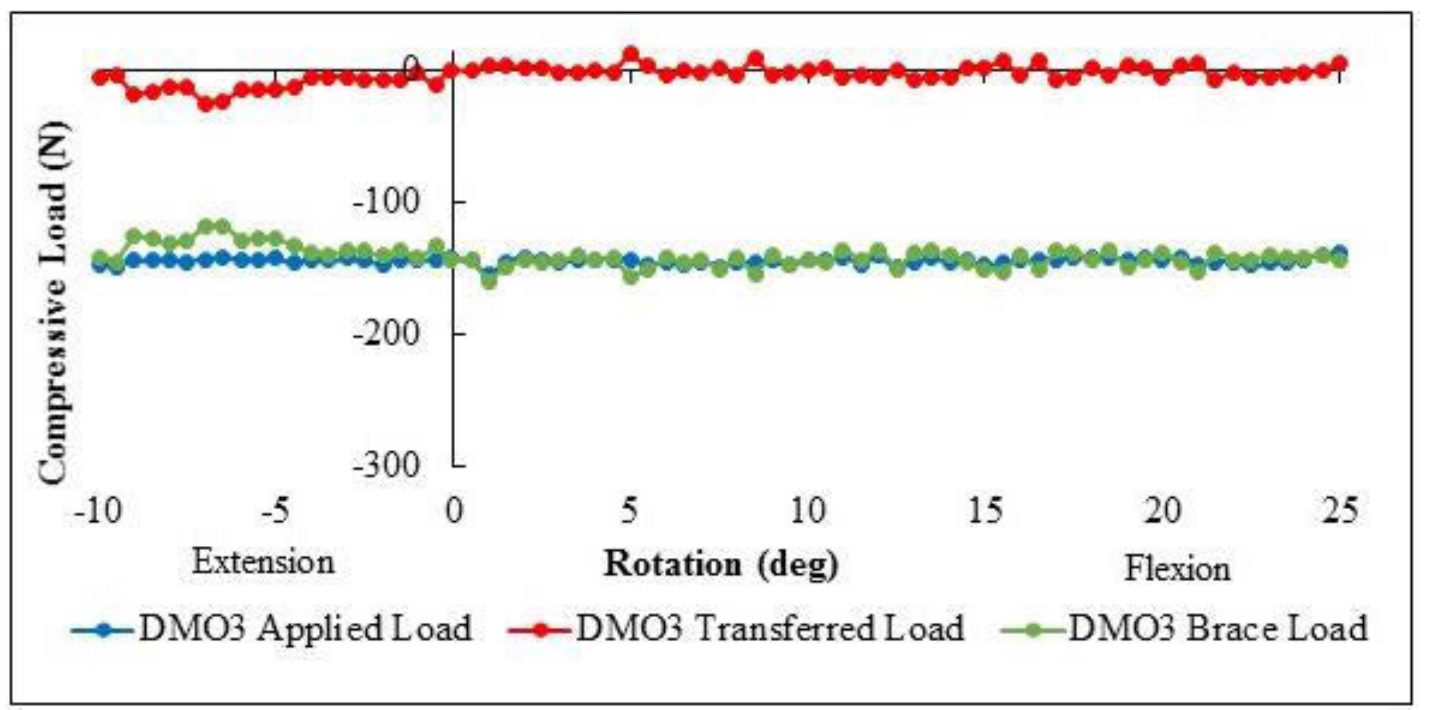

Figure 4-3. Spinal offloading of DMO3 in upright stance and throughout extended ranges of flexion and extension

Table 4-3. DMO3 compressive load values at upright stance and at end ranges of motion

\begin{tabular}{lcccc}
\hline $\begin{array}{c}\text { Degrees of } \\
\text { Rotation }\end{array}$ & $\begin{array}{c}\text { Applied } \\
\text { Load (N) }\end{array}$ & $\begin{array}{c}\text { Transferred } \\
\text { Load (N) }\end{array}$ & $\begin{array}{c}\text { Brace } \\
\text { Load (N) }\end{array}$ & $\begin{array}{c}\text { Brace Load as a } \\
\text { Percentage of } \\
\text { Applied Load (\%) }\end{array}$ \\
\hline At $10^{\circ}$ Extension & 148 & 5 & 143 & 97 \\
At $0^{\circ}$ & 143 & 0 & 143 & 100 \\
At $25^{\circ}$ Flexion & 139 & -6 & 145 & 104 \\
\hline
\end{tabular}




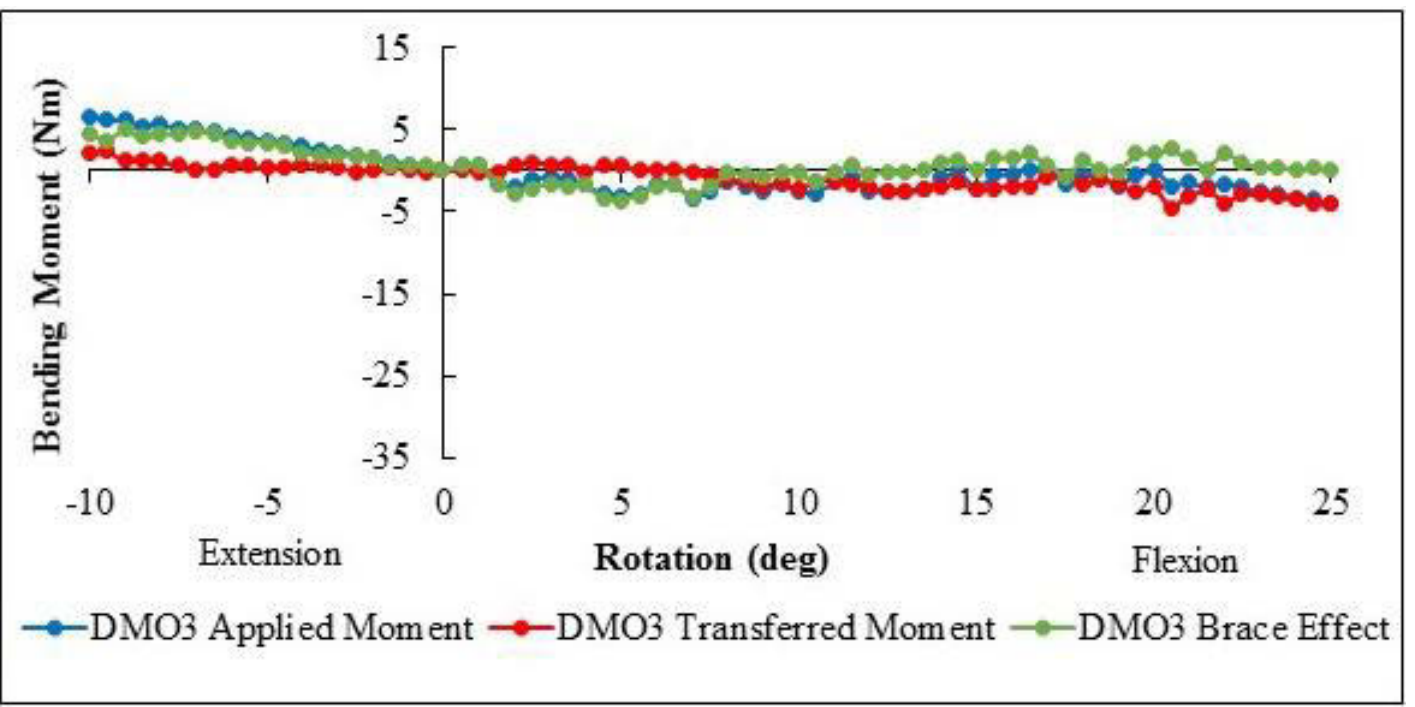

Figure 4-4. Resistance to bending of DMO3 in upright stance and throughout extended ranges of flexion and extension

Table 4-4. DMO3 bending moment values at end ranges of motion

\begin{tabular}{lcccc}
\hline $\begin{array}{c}\text { Degrees of } \\
\text { Rotation }\end{array}$ & $\begin{array}{c}\text { Applied } \\
\text { Moment } \\
(\mathbf{N m})\end{array}$ & $\begin{array}{c}\text { Transferred } \\
\text { Moment } \\
(\mathbf{N m})\end{array}$ & $\begin{array}{c}\text { Brace } \\
\text { Effect } \\
(\mathbf{N m})\end{array}$ & $\begin{array}{c}\text { Brace Effect as a } \\
\text { Percentage of } \\
\text { Applied Moment (\%) }\end{array}$ \\
\hline At $10^{\circ}$ Extension & 6.6 & 2.2 & 4.4 & $67 \%$ \\
At $25^{\circ}$ Flexion & 4.1 & 4.1 & 0 & $0 \%$ \\
\hline
\end{tabular}

Table 4-5. DMO3 axial rotation degree values for each direction of rotary turntable rotation

\begin{tabular}{lccc}
\hline \multicolumn{1}{c}{$\begin{array}{c}\text { Direction of } \\
\text { Rotation }\end{array}$} & Trial 1 & Trial 2 & Trial 3 \\
\hline Clockwise & $10^{\circ}$ & $10^{\circ}$ & $10^{\circ}$ \\
Counterclockwise & $10^{\circ}$ & $10^{\circ}$ & $10^{\circ}$ \\
\hline
\end{tabular}




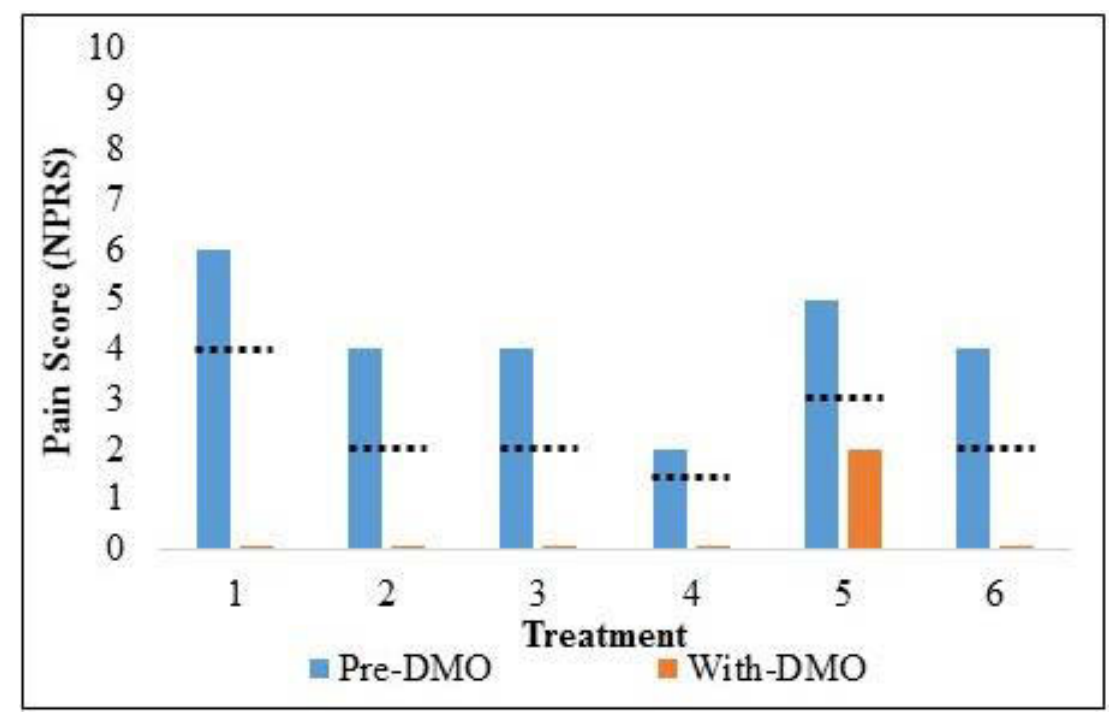

Figure 4-5. NPRS scores for patient 1 before and after wearing the DMO during visits 2 through 7

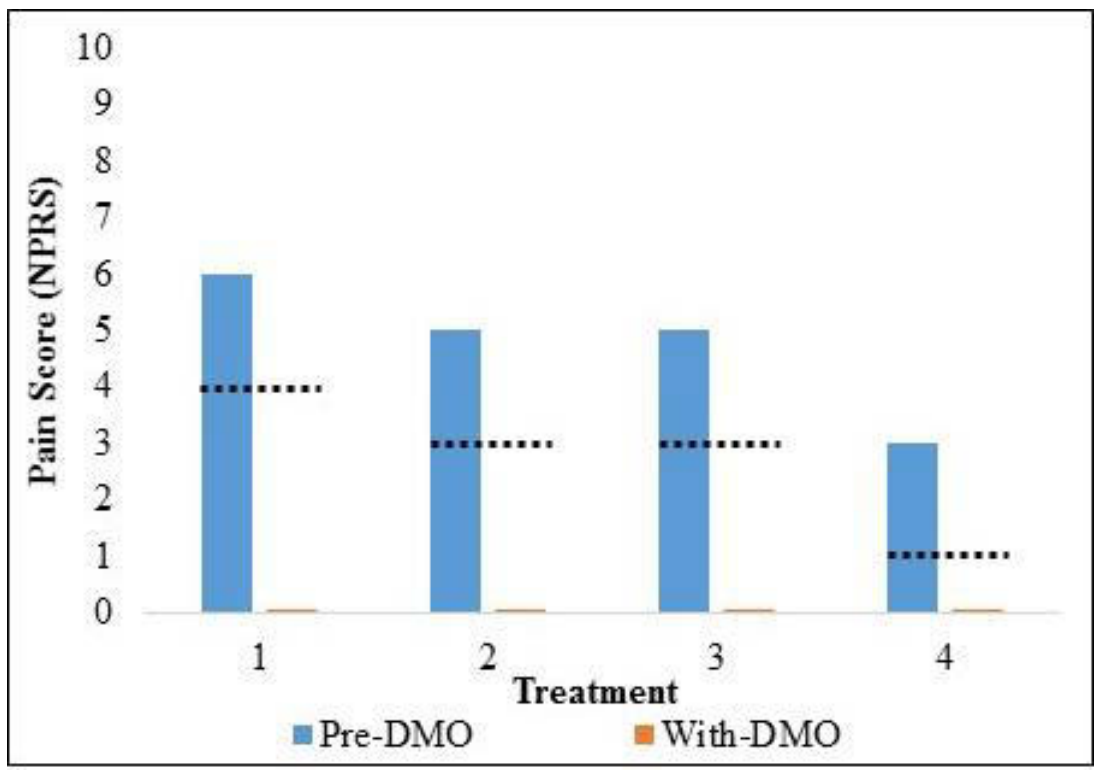

Figure 4-6. NPRS scores for patient 2 before and after wearing the DMO during visits 2 through 5 


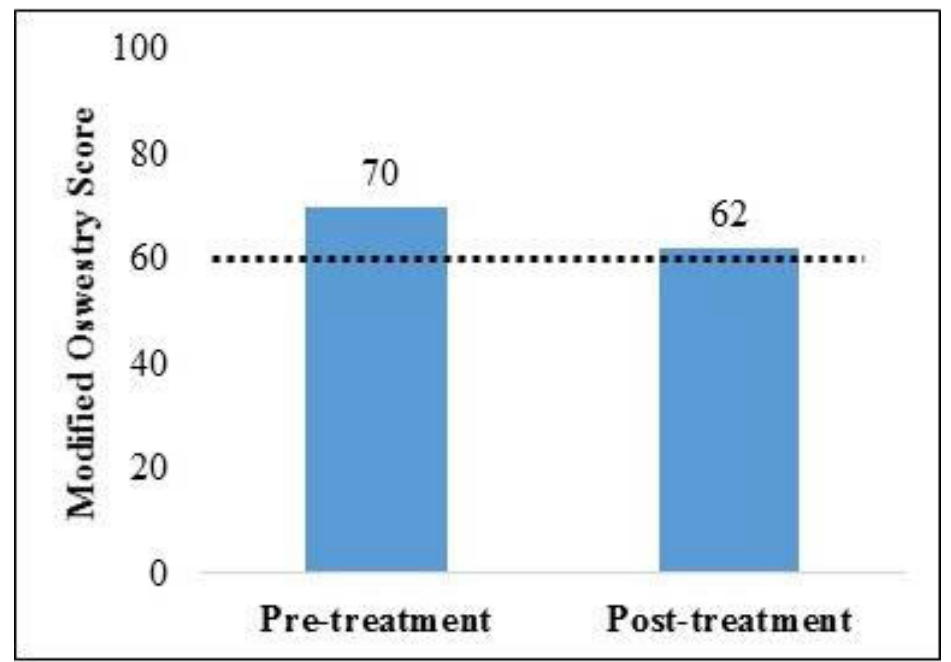

Figure 4-7. Modified Oswestry Disability Questionnaire scores for patient 1 between visit 2 and visit 7 
Figure 4-8 show the mODI scores for the patient 1 and patient 2, respectfully. For both figures, the dotted lines represent a clinically relevant decrease in mODI score [40]. The mODI score decreased from $70 \%$ to $62 \%$ for patient 1 and from $46 \%$ to $22 \%$ for patient 2, respectfully. Figure 4-9 shows lumbar disc height measurements made for patient 1 between visits 1 and 7. For patient 1, the diseased lumbar disc height at L5-S1 increased by $2 \mathrm{~mm}$ from $3.5 \mathrm{~mm}$ before treatment to $5.5 \mathrm{~mm}$ after all treatments. Figure 4-10 shows the lumbar disc height measurements made for patient 2 between visits 1 and 5 . For patient 2, the diseased lumbar disc height at L4-L5 and L5-S1 increased by $0.5 \mathrm{~mm}$ each from $6.0 \mathrm{~mm}$ before treatment to $6.5 \mathrm{~mm}$ after all treatments. 




Figure 4-8. Modified Oswestry Disability Questionnaire scores for patient 2 between visit 2 and visit 5



(a)



(b)

Figure 4-9. Lumbar disc height measurements for patient 1 between visit 1 and visit 7

(a) X-ray 1 lumbar disc height measurements during visit 1

(b) X-ray 2 lumbar disc height measurements during visit 7 


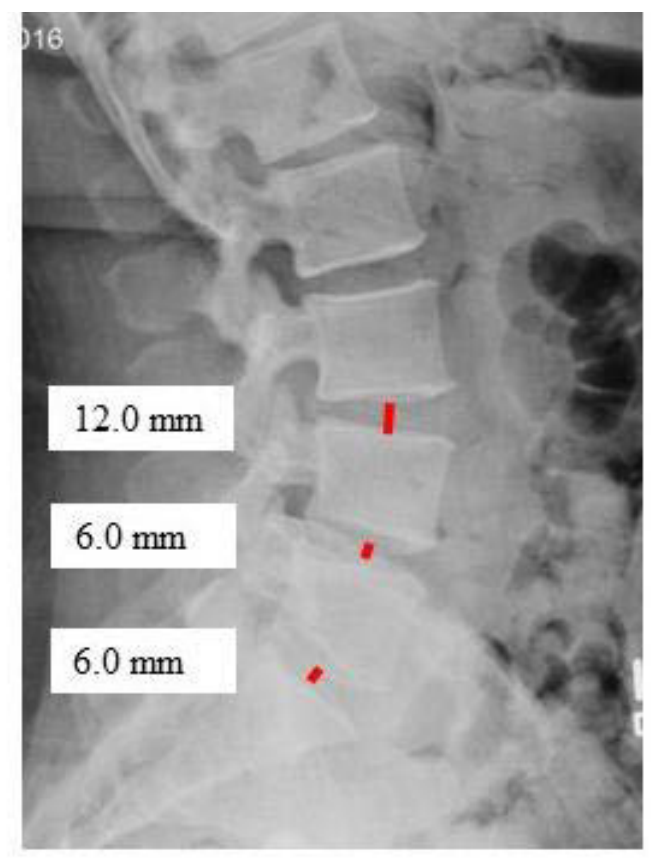

(a)

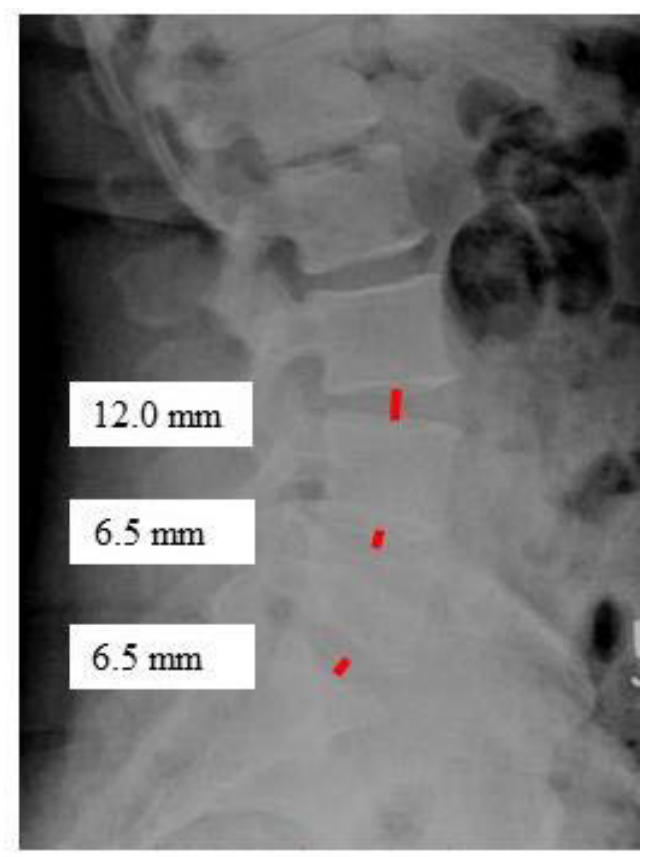

(b)

Figure 4-10. Lumbar disc height measurements for patient 2 between visit 1 and visit 5

(a) X-ray 1 lumbar disc height measurements during visit 1

(b) X-ray 2 lumbar disc height measurements during visit 5 


\section{CHAPTER 5. DISCUSSION*}

Previously examined studies both show that prolonged spinal decompression of the lumbar spine can decrease pain in patients with DDD or a disease that stems from DDD such as spinal stenosis [9-12,26]. An explanation for this may be that decompression reduces pressure on diseased discs which would promote regeneration and restoration of disc height before it began to degenerate. The decreased pressure would allow more nutrients to flow into the disc. Unfortunately, the long term effects of treatment utilizing both spinal decompression and mobility, such as with the DMO, with patients suffering from chronic LBP are not known at this time.

Lumbar intervertebral discs have a limited ability to heal post-injury most often because of the difficulty it has remodeling the large collagen fiber bundles of the AF [19]. Imaging techniques are invaluable particularly because they allow clinicians to see what the problem is and where the problem is occurring. This may seem intuitive, but this evaluation plays a large role in evaluating the best treatment method. For example, someone suffering from spondylolisthesis will likely want to stabilize their spine in hopes of restoring alignment to the lumbar vertebral column while someone suffering from spinal stenosis will likely want some sort of decompression to reduce encroachment on a spinal nerve. Without imaging to determine the cause of one's LBP, determining the appropriate treatment option could prove extremely difficult.

One of the benefits of nonsurgical treatments over surgical treatments is they are cost less. Unfortunately, nonsurgical treatment methods rely heavily on patient compliance with protocol. Lumbar orthoses are particularly intriguing in terms of treating patients with LBP. A bridging parameter, such as lumbar disc height, was used to evaluate patients suffering from DDD and spinal stenosis. The possible mechanism for the efficacy of the DMO on a patient suffering from DDD decompression would lessen disc pressure while allowing proteoglycans in the NP to increase in number to improve the disc's water-binding properties. Mobility not only promotes bulk flow of nutrients back into the intervertebral disc, but also strengthens back and abdominal muscles which promote good posture and decrease in disc pressure. All in all, the treatment of DDD is patient-specific in which imaging techniques are essential to provide successful clinical outcomes.

\section{Findings}

Minimal clinical or scientific evidence exists that supports the design rationale of Orthotrac thoracic lumbosacral orthosis was not considered to have demonstrated itself as

\footnotetext{
* Portions reprinted with permission from D. DiAngelo and D. Hillyard, "A novel distractive and mobility-enabling lumbar spinal orthosis," Journal of Rehabilitation and Assistive Technologies Engineering, vol. 3, pp. 1-10, 2016.
} 
a medically necessary device due to a shortage of peer-reviewed, placebo-controlled trials of the dynamic LSO, in particular the efficacy of back braces claiming to offload the lumbar spine. Further, from the perspective of at least one health insurance company, the [42]. More basic science and clinical studies are needed to support the claims of these dynamic spinal orthoses. The goal of this work was to address this shortcoming by designing and biomechanically evaluating novel prototypes of a dynamic LSO that provided spinal off-loading while enabling mobility.

A novel testing assembly that consisted of a custom mechanical analog torso model integrated into a robotic testing platform having advanced testing capabilities has previously been used to evaluate the loading mechanics of existing back orthoses [18], and was used in this study to carry out the design of two prototypes of a novel dynamic spinal orthosis having the unique design goal of providing spinal offloading while enabling mobility. These features, in combination, are currently not available in existing back braces on the market. Aspects of the testing protocol were selected to simulate load and movement conditions associated with many daily living activities [17].

\section{Summary of DMO2 Findings}

DMO2 was built and tested under simulated DLAs in a controlled laboratory environment. Although the original DMO1 prototype demonstrated some spinal offloading capabilities, some of the simulated vertical torso weight was transferred to the spine as flexion or extension increased. However, this outcome was not observed with the revised DMO2 model. Spinal loads were completely supported by the orthosis during upright stance and continued throughout extended ranges of flexion and extension by the DMO2 prototype. Another positive outcome of DMO2 was the ability to allow flexion and extension movement to occur with minimal bending moment buildup. The DMO1 prototype required $32.4 \mathrm{Nm}$ of moment to reach $25^{\circ}$ flexion compared with $21.7 \mathrm{Nm}$ for the DMO2 prototype. Similarly, the DMO1 prototype required $15 \mathrm{Nm}$ of moment to reach $10^{\circ}$ extension compared with $10.9 \mathrm{Nm}$ for the DMO2 prototype.

In the original DMO1 prototype a flexible rod was used to create a distractive force across the lumbar spine. However, the same rod was used as a guide to allow flexion and extension motion. Due to the uncontrolled deformation of the rod under the distractive force, the MEC of DMO1 only allowed limited unconstrained movement until the components began to bind which affected both the offloading capacity and the orthosis' resistance to bending, i.e., increased brace effect moment. By redesigning the components such that the DFC and MEC of the DMO function independently, desired spinal off-loading and bending moment values were improved. For the DMO2 prototype, the flexible rod was used only for its distractive force capabilities. By adding a nondeformable ring that had a fixed rotational axis which could be aligned to the spine's native rotational axis to provide optimal guided motion, a spinal orthosis was redesigned that met the goals of offloading the lumbar spine while enabling motion without buildup of any excessive bending moments. DMO2 functioned well in a laboratory setting; however, limitations with the design of DMO2 needed to be addressed in order to 
accomplish the design goals when the orthosis was worn on human subjects as previously outlined in the design process shown in Figure 3-1. These limitations ultimately led to the motivation into developing a third DMO prototype, DMO3. DMO2's limitations are outlined in detail below.

The first limitation was DMO2's engagement mechanism of the torso glove and the pelvic belt worked well on the human mechanical analog, but this was not the case when DMO2 was placed on human subjects. In order for DMO2 to engage properly on the wearer when a distractive force is being applied, the torso glove must not slip upwards when the DFC is engaged and the pelvic belt must not slip downwards. What was learned was that, on human subjects, the torso glove will tend to slip upwards some amount no matter how tightly the DMO is fastened to human subjects. This is primarily due to soft tissue between the DMO and the wearer's anatomy that it is attempting to engage to. It is believed that the two main factors of engagement for the torso glove are surface area and security(tightness) of the torso glove on the wearer's torso.

The second limitation was that the control of exactly how much distractive force was being applied was very arbitrary and based on feel rather than a desired force. As previously described, as the user applied a distractive force, the flexible rod deflected. The flexible rod behaved like a normal compression spring in that as it deflectd, the force applied by the flexible rod increased. Therefore, it was not possible to know how much force was being applied without adding a sensor or some sort of displacement calibration device on DMO2. Ideally, one would like to be able to target a specific distractive force for the device to apply and then have the user engage the device so that it applies that exact force. One idea to overcome this limitation was to incorporate constant force springs as part of the DFC instead of having a "spring" with variable force output as it related to its displacement.

The third and final limitation of DMO2 was that, although the location of the CoR of DMO2 MEC could be located before applying a distractive force, that location tended to change by migrating upwards when the distractive force was applied. This limitation was somewhat related to the first limitation in that it was learned that the device needed the ability to travel upwards in order to engage the torso glove on the wearer. This limitation needed to be addressed in the design of a new prototype, DMO3. It was believed that by anchoring the CoR of the MEC of DMO3 to the pelvic belt, one could approximately locate the CoR before applying a distractive force to the wearer and the $\mathrm{CoR}$ of the device would not migrate upwards like in the design of the DMO2 prototype.

\section{Summary of DMO3 Findings}

After consideration of the limitations associated with DMO2, DMO3 was built and tested under simulated DLAs in a controlled laboratory environment. Spinal loads were completely supported by the DMO3 during upright stance and continued throughout extended ranges of flexion and extension. Another positive outcome of DMO3 was the ability to allow flexion and extension movement to occur with minimal bending moment 
buildup. Throughout the development of the DMO, each prototype has demonstrated significant improvements compared to its predecessor. The DMO1 prototype required $32.4 \mathrm{Nm}$ of moment to reach $25^{\circ}$ flexion compared with $21.7 \mathrm{Nm}$ for the DMO2 prototype and $4.1 \mathrm{Nm}$ for the DMO3 prototype. Similarly, the DMO1 prototype required $15 \mathrm{Nm}$ of moment to reach $10^{\circ}$ extension compared with $10.9 \mathrm{Nm}$ for the DMO2 prototype and $6.6 \mathrm{Nm}$ for the $\mathrm{DMO} 3$ prototype.

The large decrease in applied moment values and brace effect moment values of DMO3 compared with DMO2 throughout testing ranges may lie in both the design of $\mathrm{DMO} 3$ and the location at which the instantaneous axis of rotation of the lumbar spine lies in flexion and extension. There is evidence to suggest that the instantaneous axis of rotation of the lumbar spine is located in the anterior portion of the disc during flexion and in the posterior portion of the disc during extension $[43,44]$. If it is assumed that the mechanical actuators on each lateral side of DMO3 are equidistant from the instantaneous axis of rotation in upright stance, then no additional moment is applied to the lumbar spine from DMO3. Because the RA moves closer to the anterior actuator and further from the posterior actuator during flexion, an additional moment assisting flexion is applied to the lumbar spine from the posterior actuator. Conversely, an additional moment assisting extension is applied to the lumbar spine from the anterior actuator during extension. This interaction likely results in the noticeably lower applied and brace effect moment values in DMO3 compared with DMO2.

The CFSs that provide the distractive force capabilities of the DMO3 prototype allow the force output of the DMO to remain constant even with minor fluctuations in vertical displacement as might be experienced in normal flexion and extension motions. Because the bending moment values were so minimal, it is further believed that the contribution of friction in the RA of the DMO3 prototype is minimal. It is believed that the primary bending moment contribution by the DMO3 comes from either accidental misplacement of the RA or as a result of the moving axis of rotation described above. The additional moment contribution from the actuator (posterior actuator during flexion and anterior actuator during extension) could assist the wearer's muscles during bending to allow them to not have to exert as much muscle effort while moving. The DMO3 prototype was able to achieve $10^{\circ}$ of axial rotation in both the clockwise and counterclockwise directions which simulated left axial twisting and right axial twisting, respectfully. The rod clevis spins fairly freely on the axis adjustment bar with minimal friction. The rod clevis and axis adjustment bar relationship can be thought of as a swivel joint by which the stationary axis adjustment bar provides the long axis on which each rod clevis spins on. If the wearer of the DMO3 prototype decides to axial twist to the left, the actuators on the right will lengthen slightly and the actuators on the left will want to shorten slightly. By incorporating CFSs such that the input force into the DFC is controlled by lengthening the CFSs, the actuators on both sides can lengthen or shorten as necessary without losing the distractive force provided by the DFC, theoretically. By designing the components such that the DFC and MEC function independently from each other and by having a fixed rotational axis which could be aligned to the lumbar spine's native rotational axis to provide optimal guided motion, the DMO3 prototype was designed that met the goals of offloading the lumbar spine while enabling motion without 
buildup of any excessive bending moments in both the laboratory setting and on human subjects.

The DMO3 prototype completely removed all pain from patient 1 at five of her six PT treatment visits. During the fifth treatment visit, the patient gave a pain score of 2 out of 10; however, she said that it was mainly because she was sore. She said that normally, it is difficult to distinguish between pain and soreness. Patient 1 said that the pain remained alleviated after treatment visits with DMO3 until laying down for a while and then getting back up. Also, the mODI score decreased from $70 \%$ to $62 \%$ for patient 1 , indicating a slight increase in quality of life. Although the disc height measurement showed an increase of $2 \mathrm{~mm}$ disc height at the L5-S1 level, it is likely that DMO3 alone attributed to a permanent increase in disc height that could be associated with an overall decrease in pain. It is possible that a slight increase in disc height occurred; however, future studies that involve patient compliance more than two to three times per week for only 30-40 minutes are needed to determine DMO3's effects on long-term pain and disc height. For patient 1 , what was particularly interesting was the fact that she was able to perform exercises for longer periods of time while wearing DMO3 compared with performing the exercises without wearing DMO3. Because of this, the therapist was able to push the patient to perform more difficult exercises such as performing squats and core balancing exercises on a foam cushion while standing as opposed to without a foam cushion without DMO3. This added instability in the exercises forces the patient to strengthen her core muscles more than without the instability. As stated by the patient, this added difficulty would not have been possible without DMO3. The therapist even stated that her posture was better while wearing DMO3 than without wearing DMO3 making the exercises more effective for the patient. Similarly, patient 2 experienced a complete removal of all pain while wearing DMO3 compared with having pain before wearing DMO3 at each treatment visit. Both patients experienced a clinically relevant decrease in pain every time they wore DMO3 for PT treatments. Patient 2's mODI scores decreased from $46 \%$ to $22 \%$ indicating a clinically relevant improvement in quality of life. Patient 2 experienced a slight increase in total disc height; however, her results were likely affected by her frequency at which she showed up to PT with DMO3.

The increase in disc height for both patients could be the result of a change in lordosis. In the future, lordosis measurements should be made to further examine the means by which the lumbar disc height is increasing. Many surgeons measure both the diseased lumbar discs as well as the adjacent lumbar disc to get a relative percentage in disc height. Typically, if the diseased disc is less than $50 \%$ of the adjacent, non-diseased disc, that patient would be considered a candidate for surgery. If a device such as DMO3 can decrease pain and increase disc height simultaneously, it may be possible to defer surgery for patients suffering from mechanical LBP. By doing so, the patient would save tremendous amounts of money by wearing DMO3 compared with having surgery. While competitors like the Vertetrac Ambulatory Traction System (Meditrac Ltd, TX, USA) may cost up to $\$ 1300, \mathrm{DMO} 3$ would likely cost less. Patient 1 even went as far to suggest that she wanted to buy DMO3 or a device that accomplished what DMO3 accomplished. 
The believed mechanism by which the DMO3 prototype works is that the distractive force applied by DFC results in a decreased pressure in the intervertebral discs. The combination of this decreased disc pressure with the ability for movements necessary during DLAs allows bulk flow of nutrients into and out of the disc. It is believed that this increased flow of nutrients will result in at least a temporary increase in lumbar disc height. It is the belief by the investigators that this increase in lumbar disc height will be related to a temporary decrease in pain for patients suffering from discogenic low back pain.

\section{Limitations of the Research}

As with most biomechanical studies, there were limitations with this research as it pertains to the testing of DMO2 and DMO3 under simulated flexion and extension. The testing protocol simulated the force components of the gravitational torso loading mechanics but not the corresponding in vivo spinal bending moment. The resultant bending moment was a function of the biomimetic spine's structural properties which were designed to emulate a normal healthy person [33]. Changes to the structural properties of the biomimetic spine would be needed to emulate the effects of select lumbar disease conditions, e.g., lower Durometer material could be used to model an injured/degenerative disc. Another limitation of the testing protocol was that motion while recording force and moment data were limited to the sagittal plane only. Future work will expand the capacity of the testing assembly to record force and moment data during flexion, extension, axial rotation, lateral bending, or any combination of the previously mentioned motions. Also, the physical size of the testing platform limited the extended range of motion test to $25^{\circ}$ of flexion and $10^{\circ}$ of extension. Despite this limitation, this range was more than adequate to simulate the movement conditions of many daily living activities. In the end the testing assembly was successful at demonstrating the spinal off-loading capabilities of the prototype orthoses (DMO2 and DMO3) as well as the required effort to move in the orthoses. The passive axial rotation capacity testing of DMO3 demonstrates the motion capacity for DMO3 to achieve $10^{\circ}$ of axial rotation in both directions without exceeding $0.1 \mathrm{Nm}$. The main limitation for the clinical study is the lack of availability of the patient to wear the DMO. The patient only wears the DMO for 30-40 minutes for two to three times per week, on average. Patient 2 has cancelled appointments making it difficult to obtain meaningful short-term pain relief-related results such as disc height, mODI scores, and pain differences between visit 2 and visit 7 . Because of this, the only true meaningful results for this patient will likely be that the DMO completely removes all pain from the patient while she is wearing the DMO assuming that the pain score trend continues at each treatment visit.

\section{Future Work}

Future research design plans for the distractive mobility-enabling orthosis are to expand the orthosis' range of movement to include lateral bending as well as to adjust location of some of the DFC components without hindering its function. To complete the current clinical protocol, a total of $10-15$ patients will be recruited. When more patients 
have been recruited, a paired t-test will be applied to test whether there was a significant (alpha $=0.05$ ) decrease in NPRS score, a decrease in mODI score, and an increase in disc height between the first and final treatment visits for each patient. A linear regression will be applied to analyze the correlation between an increase in lumbar disc height and a decrease in NPRS score. The Pearson's correlation coefficient will be determined to determine clinical significance with an alpha value of 0.05 . This data will provide necessary evidence to evaluate DMO3's short-term effects on LBP and quality of life.

Also, a larger clinical study will be undertaken on patients with mechanical LBP to prove the efficacy of this novel LSO to relieve pain for a short period of time even without wearing the DMO. The clinical trial will be done with the reconfigured DFC as shown in Figure 3-14 and Figure 3-22. Because this is essentially a pilot study, the trend of the current results indicate that a future study is warranted that will require a LBP patient to take home the DMO as prescribed by his or her doctor. It is likely that it will be prescribed to be worn for 45 minutes to an hour for a total of 4-5 days per week. Followup periods to evaluate the effectiveness of the DMO to relieve pain and improve quality of life will likely be one month, three months, six months, and one-year. A study of this magnitude would assuredly provide evidence of the DMO's short-term and long-term effectiveness for those patients suffering LBP as a result of mechanical conditions like DDD, spinal stenosis, or even vertebral body burst fractures. The combination of providing distraction across the lumbar spine with the ability to undergo controlled movement with minimal resistance could result in improved core stability and development of motor control to improve coordination of postural muscles. The possible increased lumbar disc height while wearing DMO could further lessen the effects of pain and could result in a temporary increase in lumbar disc height. Lastly, the DMO is unique in that it can provide spinal-offloading while still allowing the user to perform normal daily living activities unlike previously mentioned proven conservative treatment options such as water therapy. Also, further tests should be done by which the DFC is activated to provide a distractive force and the force response is actively measured in the RTP under simulated axial twisting.

In the future, one should consider if the lumbar spine is truly flexing $25^{\circ}$ during flexion and $10^{\circ}$ during extension with and without wearing DMO3. This could be done radiographically by measuring the rotation of $\mathrm{L} 1$ versus the rotation of the sacrum under simulated flexion and extension. In other bracing studies, a placebo effect has been considered. In the future, a study to see if there is truly a placebo effect in regards to pain decreases should be done. For example, this could be accomplished by having two groups of patients undergo treatments with DMO3. The first group would undergo treatments with DMO3 engaged, and the second group would undergo treatments without DMO3 engaged. Similar outcome measures could be taken such a NPRS scores and mODI scores to compare between groups. Fully understanding what allows DMO3 to alleviate pain is very important in the future. 


\section{Conclusion}

The combination of the mechanical analog of a life-size human upper torso and an advanced testing protocol served as a design tool to redesign a novel lumbar spinal orthosis that provided distractive forces across the lumbar spine and required minimal effort for movement. This testing assembly can also serve as the foundation for the development of new testing methods for classifying and ranking spinal orthoses. The newly designed orthosis provided the capability to apply a distractive force to the wearer without losing its output during flexion or extension (and likely axial rotation) by utilizing CFSs as the force input into the system. Another orthosis, such as the DMO, that provides a distractive force while allowing mobility currently does not exist.

Furthermore, this orthosis was able to meet the specifically chosen design goals such that it properly engaged the wearer, provided a desirable, constant distractive force, allowed mobility, and contained adjustment mechanisms to allow it to be worn by wearers of different sizes. The laboratory testing ensured that design goals were met, and the preliminary clinical testing provided promising evidence of the dynamic LSO which warrants further investigation by continuing the current clinical protocol and, eventually, organizing a larger study in which human subjects are prescribed the orthosis for a few months and the orthosis is worn at home. 


\section{LIST OF REFERENCES}

[1] J. Fritz, "Physical therapy for acute low back pain: associations with subsequent healthcare costs," Spine, vol. 33, pp. 1800-1805, 2008.

[2] D. Akay, "Ant colony optimization approach for classification of occupational low back disorder risks," Hum Factors Ergon Manuf, vol. 19, pp. 1-14, 2008.

[3] L. Brecher, "Editor's Message," JAOA, vol. 101, p. 1, 2001.

[4] P. Pensri, "Biopsychological Factors and Perceived Disability in Saleswomen with Concurrent Low Back Pain," Saf Health Work, vol. 1, pp. 149-157, 2010.

[5] G. Andersson, "Epidemiologic features of chronic low-back pain," Lancet, vol. 354, pp. 581-585, 1999.

[6] R. Deyo, "Low back pain," N Engl J Med, vol. 344, pp. 363-370, 2001.

[7] S. Bigos, "Acute low back problems in adults. Clinical practice guidelines Number 14," P. H. S. US Department of Health and Human Services, Agency for Health Care Policy and Research, Ed., ed, 1994.

[8] A. Tosteson, "The Cost Effectiveness of Surgical versus Non-Operative Treatment for Lumbar Disc Herniation over Two Years: Evidence from the Spine Patient Outcomes Research Trial (SPORT)," Spine, vol. 33, pp. 2108-2115, 2008.

[9] P. Baena-Beato, "Effects of different frequencies (2-3 days/week) of aquatic therapy program in adults with chronic low back pain. A non-randomized comparison trial," Pain Med, vol. 14, pp. 145-158, 2013.

[10] A. Cuesta-Vargas, "Deep water running and general practice in primary care for non-specific low back pain versus general practice alone: randomized controlled trial," Clin Rheumatol, vol. 31, pp. 1073-1078, 2012.

[11] U. Dundar, "Clinical effectiveness of aquatic exercise to treat chronic low back pain: a randomized controlled trial," Spine, vol. 34, pp. 1436-1440, 2009.

[12] Y. Mirovsky, "The effect of ambulatory lumbar traction combined with treadmill on patients with chronic low back pain," J Back Musculoskelet Rehabil, vol. 19, pp. 73-78, 2006.

[13] M. Krag, "Comparison of three lumbar orthoses using motion assessment during task performance," Spine, vol. 28, pp. 2359-2367, 2003.

[14] S. Lantz, "Lumbar spine orthosis wearing. I. Restriction of gross body motions," Spine, vol. 11, pp. 834-837, 1986.

[15] L. Ferrara, "A biomechanical assessment of disc pressures in the lumbosacral spine in response to external unloading forces," Spine J, vol. 5, pp. 548-553, 2005.

[16] D. DiAngelo, "Towards the Design of a Distractive and Mobility-Enabling Back Support Device," Journal of Mississippi Academy of Sciences, vol. 31, pp. 193200, 2015.

[17] J. Bible, "Normal functional range of motion of the lumbar spine during 15 activities of daily living," J Spinal Disord Tech, vol. 23, pp. 106-112, 2010.

[18] D. DiAngelo, "Robotized Method for Comparative Testing of Back Support Devices," Journal of Mississippi Academy of Sciences, vol. 31, pp. 179-186, 2015. 
[19] M. Adams, The Biomechanics of Back Pain vol. 1. Edinburgh, Scotland: Elsevier Science Ltd, 2002.

[20] M. Brown, "Sensory and sympathetic innveration of the vertebral endplate in patients with degenerative disc disease," J Bone Joint Surg, vol. 79, pp. 147-53, 1997.

[21] M. Modic, "Lumbar Degenerative Disk Disease," Radiology, vol. 245, pp. 43-61, 2007.

[22] D. Hoy, "The global burden of low back pain: estimates from the Global Burden Disease 2010 Study," Ann Rheum Dis, vol. 73, pp. 968-974, 2014.

[23] G. Jensen, "Biomechanics of the lumbar intervertebral disk: a review," Phys Ther, vol. 60, pp. 765-773, 1980.

[24] B. Koes, "Diagnosis and treatment of low back pain," BMJ, vol. 332, pp. 14301434, 2006.

[25] R. Chou, "Low back pain (chronic)," BMJ Clinical Evidence, vol. 10, pp. 1-41, 2009.

[26] C. Apfel, "Restoration of disk height through non-surgical spinal decompression is associated with decrease discogenic low back pain: a retrospective cohort study," BMC Muscluoskelet Disord, vol. 11, pp. 1-6, 2010.

[27] P. Fritzell, "2001 Volvo Award Winner in Clinical Studies: Lumbar fusion versus nonsurgical treatment for chronic low back pain: a multicenter randomized controlled trial from the Swedish Lumbar Spine Study Group," Spine, vol. 26, pp. 2521-2532, 2001.

[28] J. Brox, "Randomized clinical trial of lumbar instrumented fusion and cognitive intervention and exercises in patients with chronic low back pain and disc degeneration," Spine, vol. 28, pp. 1913-1921, 2003.

[29] G. Kawchuk, "A non-randomized clinical trial to assess the impact of nonrigid, inelastic corsets on spine function in low back pain participants and asymptomatic controls," Spine J, vol. 15, pp. 2222-2227, 2015.

[30] D. Johnson, "Active Spinal Orthosis to Reduce Lumbar Postural Muscle Activity in Flexed Postures," JPO, vol. 28, pp. 109-113, 2016.

[31] J. Cannon, "Evidence on the Ability of a Pneumatic Decompression Belt to Restore Spinal Height Following an Acute Bout of Exercise," JMPT, vol. 39, pp. 304-310, 2016.

[32] P. Leake. vertecorelift. Available: https://vertecorelift.wordpress.com/howvertecore-lift-works/

[33] J. Simmons, "Development of a Mobility-Enabling Spinal Orthosis and Methods for Evaluating and Developing Spinal Orthoses on a Robotic Platform," PhD, UTHSC Orthopedic Surgery \& Biomedical Engineering, The University of Tennessee Health Science Center, 2014.

[34] D. Winter, Biomechanics of Human Movement. New York: Wiley, 1979.

[35] J. Stubbs, "Use of a multi-axis robotic testing platform to investigate the sagittal mechanics of the multi-body lumbar spine," Master of Science, Department of Orthopaedic Surgery and Biomedical Engineering, The University of Tennessee Health Science Center, 2014. 
[36] B. Kelly, "A Multiaxis Programmable Robot for the Study of Multibody Spine Biomechanics Using a Real-Time Trajectory Path Modification Force and Displacement Control Strategy," J Med Devices, vol. 7, pp. 1-7, 2013.

[37] I. Gilad, "A study of vertebra and disc geometric relations of the human cervical and lumbar spine," Spine, vol. 11, pp. 154-157, 1986.

[38] J. Crisco, "Optimal marker placement for calculating the instantaneous center of rotation," J Biomech, vol. 27, pp. 1183-1187, 1994.

[39] J. Childs, "Responsiveness of the Numeric Pain Rating Scale in Patient with Low Back Pain," Spine, vol. 30, pp. 1331-1334, 2005.

[40] O. Hägg, "The clinical importance of changes in outcome scores after treatment for chronic low back pain," Eur Spine J, vol. 12, pp. 12-20, 2003.

[41] D. DiAngelo and D. Hillyard, "A novel distractive and mobility-enabling lumbar spinal orthosis," Journal of Rehabilitation and Assistive Technologies Engineering, vol. 3, pp. 1-10, 2016.

[42] (2013). Medical Coverage Policy: Thoracic Lumbosacral Orthosis with Pneumatics.

[43] A. W. III, Clinical Biomechanics of the Spine, 2nd ed. Philadelphia: J.B. Lippincot Company, 1990.

[44] D. Sengupta, "Instant axis of rotation of L4-5 motion segment--a biomechanical study on cadaver lumbar spine," J Indian MEd Assoc, vol. 109, pp. 389-395, 2011. 


\section{VITA}

Daniel Clay Hillyard was born in Memphis, TN in 1991 and raised in Bartlett, TN. He graduated from Bartlett High School in 2010 and from The University of Tennessee - Knoxville in 2014 with a Bachelor of Science in Honors Biomedical Engineering. From 2011-2014, he worked in BioRobotics Laboratory at The University of Tennessee Health Science Center as a part of the Summer Research Scholars internship program which was sponsored by the College of Graduate Health Sciences at The University of Tennessee Health Science Center in Memphis. Through his time in the Summer Research Scholars Program, he helped with many projects throughout the lab including aiding in the development of the initial distractive and mobility-enabling orthosis prototype. His passion for medical device design and orthopedic research coupled with his positive experience with laboratory personnel ultimately led to him joining the BioRobotics Laboratory at the University of Tennessee Health Science Center in August 2014. 